JOURNAL OF

SYMPLECTIC GEOMETRY

Volume 10, Number 1, 81-164, 2012

\title{
PERIODIC FLOER HOMOLOGY AND SEIBERG-WITTEN-FLOER COHOMOLOGY
}

\author{
Yi-Jen Lee and Clifford Henry Taubes
}

\begin{abstract}
Various Seiberg-Witten-Floer cohomologies are defined for a closed, oriented three-manifold; and if it is the mapping torus of an areapreserving surface automorphism, it has an associated periodic Floer homology as defined by Michael Hutchings. We construct an isomorphism between a certain version of Seiberg-Witten-Floer cohomology and the corresponding periodic Floer homology, and describe some immediate consequences.
\end{abstract}

\section{Introduction}

Suppose that $F$ is a closed, oriented two-manifold with an area form and a given volume-preserving diffeomorphism $f: F \rightarrow F$. Let $M$ denote the threemanifold $(\mathbb{R} \times F) / \mathbb{Z}$ where the $\mathbb{Z}$-action has 1 sending any given $(t, x) \in \mathbb{R} \times F$ to $(t+2 \pi, f(x))$. Michael Hutchings $[\mathbf{H 1}, \mathbf{H S}]$ defined a version of Floer homology in this context which he called periodic Floer homology. To a first approximation, the chain complex is generated by sets of pairs where each pair consists of an irreducible periodic orbit of $f$ and a positive integer. The differential is defined using pseudoholomorphic curves in $\mathbb{R} \times M$ as defined by an appropriately chosen, $\mathbb{R}$-invariant almost complex structure. Hutchings conjectured that this periodic Floer homology is isomorphic to a version of the Seiberg-Witten-Floer cohomology. The purpose of this article is to explain how the analysis used in $[\mathbf{T 1}, \mathbf{T 2}, \mathbf{T 3}, \mathbf{T 4}]$ to establish the equivalence between Seiberg-Witten-Floer cohomology and embedded contact homology can be used to prove Hutchings's conjectured equivalence between periodic Floer homology to Seiberg-Witten-Floer cohomology. A precise statement of the equivalence is given in Section 1.3 for the monotone case. The general version of the isomorphism theorem requires additional preparation to state, and is therefore postponed until Section 6.3. What follows directly sets the stage with a more detailed description of periodic Floer homology and of the relevant version of Seiberg-Witten-Floer cohomology. 
1.1. Periodic Floer homology: the monotone case. The description that follows of periodic Floer homology for the most part paraphrases what is presented in $[\mathbf{H} \mathbf{1}, \mathbf{H S}]$.

1.1.1. The Geometry of $\boldsymbol{M}$. The projection map from $\mathbb{R} \times F$ to $\mathbb{R}$ descends to $M$ so as to give a fibration $\pi: M \rightarrow S^{1}$. Here, $S^{1}$ is identified with $\mathbb{R} /(2 \pi \mathbb{Z})$. Conversely, a closed, oriented three-manifold that fibers over $S^{1}$ admits a fiber-preserving diffeomorphism to the manifold $(\mathbb{R} \times F) / \mathbb{Z}$, where $F$ here is the fiber of the fibration, and where the $\mathbb{Z}$-action is defined by the area-preserving diffeomorphism, $f$, of $F$.

The push forward of the Euclidean vector field on $\mathbb{R}$ defines a nowhere zero vector field, $\partial_{t}$, which is transverse to the fibers of $\pi$. Closed integral curves of $\partial_{t}$ are determined by the fixed points of the iterates of $f$. To elaborate, introduce the notion of a periodic orbit of $f$. This is a finite, cyclically ordered set of distinct points in $F$ that are cyclically permuted by $f$. Let $\gamma \subset F$ denote such a set. The number of points in $\gamma$ is said to be the period. The periodic orbits of $f$ are in 1-1 correspondence with the compact, embedded one-dimensional submanifolds that are integral curves of $\partial_{t}$. The symbol $\gamma$ is used in what follows to denote both a periodic orbit and also the associated embedded integral curve of $\partial_{t}$.

A periodic orbit of period $q$ is said to be non-degenerate when the linear map $1-d\left(f^{k q}\right)$ on $\left.T F\right|_{\gamma}$ is invertible for all integers $k>0$. Here, $f^{m}$ is shorthand for the $m$ th iterate of the map $f$. We assume in what follows that

$$
f \text { is chosen so that all orbits are non-degenerate. }
$$

An orbit is said to be hyperbolic if $d f^{q}$ has real eigenvalues. Otherwise the orbit is said to be elliptic. The hyperbolic orbit is positive or negative when the eigenvalues of $d f^{q}$ are, respectively, positive or negative.

Any given closed integral curve of $\partial_{t}$ has a canonical orientation, namely the orientation along $\partial_{t}$. This understood, such an integral curve defines a class in $H_{1}(M ; \mathbb{Z})$. If $\gamma$ denotes the integral curve, then $[\gamma]$ will denote the corresponding homology class. Meanwhile, the fiber $F$ of $\pi$ is oriented by its area form and so defines a class, $[F]$, in $H_{2}(M ; \mathbb{Z})$. The Poincaré dual of this class pairs with $[\gamma]$ to give the period of the corresponding periodic orbit of $f$.

Two other cohomology classes play a distinguished role in what follows. To describe the first of these, introduce $w_{F}$ to denote the area form of the surface $F$. Since $f$ preserves $w_{F}$, this form descends to $M$ as a closed, nowhere zero 2-form on $M$ that annihilates the vector field $\partial_{t}$. We denote this twoform on $M$ by $w_{f}$; and use $\left[w_{f}\right]$ to denote its cohomology class in $H^{2}(M ; \mathbb{R})$. The second is the Euler class of the kernel of $\pi$ 's differential. This class is denoted as $c_{1}\left(K^{-1}\right)$. Note that while the class $c_{1}\left(K^{-1}\right)$ remains fixed under symplectic isotopies of $f$, the class $\left[w_{f}\right]$ varies in the following manner: By 
the Mayer-Vietoris sequence, we have the following exact sequence:

$$
0 \rightarrow\left(\left.\operatorname{Ker}\left(1-f_{*}\right)\right|_{H_{1}(F)}\right)^{*} \rightarrow H^{2}(M ; \mathbb{R}) \rightarrow(\mathbb{R}[F])^{*} \rightarrow 0 .
$$

By the definition of $w_{f}$, the cohomology class $\left[w_{f}\right]$ belongs to the coset of elements in $H^{2}(M)$ which maps to $\left[w_{F}\right] \in H^{2}(F)$. As $f$ varies through a symplectic isotopy, the class $\left[w_{f}\right]$ varies by the image of the flux of this symplectic isotopy under the composite map: $H^{1}(F) \rightarrow\left(\left.\operatorname{Ker}\left(1-f_{*}\right)\right|_{H_{1}(F)}\right)^{*} \rightarrow$ $H^{2}(M ; \mathbb{R})$. Thus, $\left[w_{f}\right]$ is invariant under Hamiltonian isotopies of $f$, and, because of the surjectivity of the flux homomorphism, it can take values in any element in the half space $\{e \mid\langle e,[F]\rangle>0\} \subset H^{2}(M ; \mathbb{R})$ under symplectic isotopies and rescaling.

Definition 1.1. Given a class $\Gamma \in H_{1}(M ; \mathbb{Z})$, Let $e_{\Gamma}$ denote the Poincaré dual of $\Gamma$, and let

$$
c_{\Gamma}:=2 e_{\Gamma}+c_{1}\left(K^{-1}\right) .
$$

The class $\Gamma$ is said to be monotone (with respect to $\left[w_{f}\right]$ ) if

$$
\left[w_{f}\right]=-\lambda c_{\Gamma} \quad \text { for some } \lambda \in \mathbb{R} \text {. }
$$

Note that since $\left[w_{f}\right]$ is non-trivial, by this definition $c_{\Gamma}$ cannot be torsion if $\Gamma$ is monotone, and $\lambda \neq 0$. We call $\Gamma$ positive monotone when $\lambda>0$; conversely, negative monotone if $\lambda<0$.

Fix a monotone $\Gamma$ throughout this subsection.

Let

$$
d_{\Gamma}:=e_{\Gamma}([F]) .
$$

Note that since $c_{\Gamma}$ is non-torsion, $d_{\Gamma} \neq$ genus $(F)-1$. This condition guarantees that any given map $f$ can be isotoped via a symplectic isotopy to obtain monotonicity. (A generic map $f$ will not have any monotone classes.)

1.1.2. The chain complex. Introduce the set $\mathcal{A}$ whose elements are described next. A given element, $\Theta$, is a finite set of pairs, each of the form $(\gamma, m)$ where $\gamma$ is a periodic orbit of $f$ and where $m$ is a positive integer. These are constrained as follows:

$$
\left\{\begin{array}{l}
\text { - } \sum_{(\gamma, m) \in \Theta} m[\gamma]=\Gamma \text {. } \\
\text { - Distinct pairs have distinct periodic orbit components. } \\
\text { - The integer } m=1 \text { when } \gamma \text { is hyperbolic. }
\end{array}\right.
$$

Note that $\Theta$ can be the empty set, and that $\mathcal{A}$ is a finite set. Indeed, such is the case because the integer $d_{\Gamma}$ is the sum over each $(\gamma, m)$ in $\Theta$ of $m$ times the period of $\gamma$. Meanwhile, there are only finitely many periodic points with period bounded by any given integer. Let $\mathcal{A}_{+}$denote the set of pairs of the form $(\Theta, \mathfrak{o})$ where $\Theta \in \mathcal{A}$ and where $\mathfrak{o}$ denotes an ordering of the elements in $\Theta$ whose periodic orbit component is positive and hyperbolic. The chain complex for periodic Floer homology is defined to be

$$
C P_{*}(f, \Gamma)=\mathbb{Z} \mathcal{A}_{+} / \sim
$$


where $\sim$ denotes the equivalence relation that has $(\Theta, \mathfrak{o}) \sim-\left(\Theta, \mathfrak{o}^{\prime}\right)$ when $\mathfrak{o}^{\prime}$ differs from $\mathfrak{o}$ by an odd permutation. This is a free $\mathbb{Z}$-module. One can assign to each $\Theta \in \mathcal{A}$ a fixed ordering, $\mathfrak{o}$, of the elements in $\Theta$ whose periodic orbit component is positive and hyperbolic. Doing so identifies the chain complex $C P_{*}$ with $\mathbb{Z} \mathcal{A}$. Such an assignment is assumed implicitly in what follows unless stated to the contrary. This the case, each $\Theta \in \mathcal{A}$ can be viewed as a generator of the chain complex.

1.1.3. A relative grading. The chain complex has a relative $\mathbb{Z} / p \mathbb{Z}$-grading where $p$ is the divisibility in $H^{2}(M ; \mathbb{Z}) /$ Tors of the class $c_{\Gamma}$. To define this grading, suppose that $\Theta_{+}$and $\Theta_{-}$are two elements from $\mathcal{A}$. Use $H_{2}\left(M ; \Theta_{+}, \Theta_{-}\right)$to denote the set of relative homology classes of integervalued two-chains $Z \subset M$ with

$$
\partial Z=\sum_{(\gamma, m) \in \Theta_{+}} m \gamma-\sum_{(\gamma, m) \in \Theta_{-}} m \gamma .
$$

This is a torsor modeled on $H_{2}(M ; \mathbb{Z})$. Any given $Z \in H_{2}\left(M ; \Theta_{+}, \Theta_{-}\right)$can be used to associate an integer, $I\left(\Theta_{+}, \Theta_{-} ; Z\right)$, to the pair $\left(\Theta_{+}, \Theta_{-}\right)$. This integer is defined in Section 2 of $[\mathbf{H 1}]$. As explained in the latter reference, this integer has two nice features.

(1) $I\left(\Theta_{+}, \Theta_{-} ; Z\right)$ changes by a multiple of $p$ when the class $Z$ is changed. More precisely,

$$
I\left(\Theta_{+}, \Theta_{-} ; Z\right)-I\left(\Theta_{+}, \Theta_{-} ; Z^{\prime}\right)=\left\langle c_{\Gamma},\left[Z-Z^{\prime}\right]\right\rangle .
$$

(2) $I\left(\Theta, \Theta^{\prime} ; Z\right)+I\left(\Theta^{\prime}, \Theta^{\prime \prime} ; Z^{\prime}\right)=I\left(\Theta, \Theta^{\prime \prime} ; Z+Z^{\prime}\right)$.

These two features imply that

$$
I\left(\Theta_{+}, \Theta_{-}\right):=I\left(\Theta_{+}, \Theta_{-} ; \cdot\right) \bmod p \mathbb{Z}
$$

gives a relative $\mathbb{Z} / p \mathbb{Z}$-grading to the chain complex.

Some digression is required before introducing the differential.

1.1.4. The set of complex structures $\mathcal{J}_{\boldsymbol{f}}$. Let $\mathcal{J}_{f}$ be the set of almost complex structures $J$ on $\mathbb{R} \times M$ satisfying the following properties:

(1) $J$ is invariant with respect to the action of $\mathbb{R}$ on $\mathbb{R} \times M$ that translates a constant amount along the $\mathbb{R}$ factor.

(2) Let $s$ denote the Euclidean coordinate along the $\mathbb{R}$ factor in $\mathbb{R} \times M$. Then $J \cdot \partial_{s}=\partial_{t}$.

(3) Let $\gamma$ denote any periodic orbit from a set in $\mathcal{A}$. View $\gamma$ as an embedded circle in $M$. Then $\left.J\right|_{\gamma}$ maps $\operatorname{Ker}\left(\pi_{*}\right)$ to itself.

(4) $J$ defines a tame almost complex structure with respect to the symplectic form

$$
\Omega_{F}=d s \wedge d t+w_{f}
$$

on $\mathbb{R} \times M$. That is, the quadratic form $\Omega_{F}(\cdot, J(\cdot))$ on $T(\mathbb{R} \times M)$ is positive. 
Endow $\mathcal{J}_{f}$ with the $C^{\infty}$-Frechet topology. We say that $J$ is admissible if condition (3) above is replaced by the stronger condition:

$\left(3^{\prime}\right) J$ maps Ker $\pi_{*}$ to itself at any point on $M$.

1.1.5. Pseudoholomorphic subvarieties in $\mathbb{R} \times M$. For the purposes of this article, a pseudoholomorphic subvariety in $\mathbb{R} \times M$ is a closed subset $C \subset \mathbb{R} \times M$ with the following properties:

- $C$ has no point components.

- The complement in $C$ of a finite set of points is a smooth, nonempty, two-dimensional submanifold of $\mathbb{R} \times M$ whose tangent space is $J$-invariant. Endow $C$ with the complex structure and orientation induced from $J$ away from these points.

- The integral over $C$ of $w_{f}$ is finite.

Remark. The third item of this definition is equivalent to the requirement that $C$ has finite energy with respect to the symplectic form $\Omega_{F}$ in the sense of Hofer et al. (cf., e.g., Section 5.3 in [BEHWZ]). Indeed, the integral $\int_{C} w_{f}$ is what is termed " $\omega$-energy" in the aforementioned reference. On the other hand, the integral $\int_{C \cap\{s\} \times M} d t$ is independent of $s$ whenever it is welldefined, since slices of $C$ at any $s \in \mathbb{R}$ are all homologous in $M$. Denote this constant by $l$. (In our context, which will be described momentarily, $l=d_{\Gamma}$ ). Then

$$
\int_{C \cap[s-L, s+L] \times M} d s \wedge d t=2 L l \quad \forall s \in \mathbb{R} .
$$

This implies that the " $\lambda$-energy" in $[\mathbf{B E H W Z}]$ is bounded automatically.

To continue the digression, if $\gamma \subset M$ is a closed integral curve of $\partial_{t}$, then the cylinder $\mathbb{R} \times \gamma \subset \mathbb{R} \times M$ is pseudoholomorphic. Cylinders of this sort are the only $\mathbb{R}$-invariant, irreducible pseudoholomorphic subvarieties. (A pseudoholomorphic subvariety is said to be irreducible when no finite set has disconnected complement.)

Suppose that $C$ is a pseudoholomorphic subvariety. The condition that $w_{f}$ have finite integral on $C$ can be used to show the following: There exists $s_{0} \in \mathbb{R}$ such that the $|s| \geq s_{0}$ portion of $C$ is a disjoint union of embedded submanifolds on which $s$ restricts with no critical points. In particular, each such submanifold is a cylinder that lies in a small radius tubular neighborhood of either the $s \geq s_{0}$ or $s \leq-s_{0}$ part of one of the $\mathbb{R}$-invariant, pseudoholomorphic cylinders, thus a in a neighborhood of $\mathbb{R} \times \gamma$ with $\gamma \subset M$ being a closed, integral curve of $\partial_{t}$. These cylinders are called the ends of $C$. An end where $s$ is unbounded from below is said to be a negative end, and one where $\mathrm{s}$ is unbounded from above is said to be a positive end.

The constant- $s$ slices of any given end of $C$ limit as $|s| \rightarrow \infty$ to a closed integral curve of $\partial_{t}$. To elaborate, let $\gamma \in M$ denote such a curve. The nondegeneracy of $\gamma$ implies the following: There exists a disk $D \subset \mathbb{C}$ about the 
origin and an embedding $\varphi_{\gamma}: S^{1} \times D \rightarrow M$ such that $\varphi_{\gamma}(\cdot, 0)=\gamma$, with the additional properties described below: Suppose first that $\mathcal{E} \subset C$ is a positive end whose constant $s$ slices limit to $\gamma$ as $s \rightarrow \infty$. Let $\hat{\varphi}_{\gamma}$ denote the map id $\times \varphi_{\gamma}: \mathbb{R} \times\left(S^{1} \times D\right) \rightarrow \mathbb{R} \times M$. Then there exists $s_{\mathcal{E}}>0$ and an integer $q_{\mathcal{E}} \geq 1$, such that $\hat{\varphi}_{\gamma}^{-1}\left(\mathcal{E} \cap\left(\left[s_{\mathcal{E}}, \infty\right) \times M\right)\right)$ is the image of a map from $\left[s_{\mathcal{E}}, \infty\right) \times \mathbb{R} / 2 \pi q_{\mathcal{E}} \mathbb{Z}$ to $\mathbb{R} \times\left(S^{1} \times D\right)$ that has the form $(s, \tau) \mapsto(s,(\tau, \varsigma(\tau, s))$, where $\varsigma$ can be written as

$$
\varsigma(\tau, s)=\mathrm{e}^{-\lambda_{q_{\mathcal{E}}} s} \varsigma_{q_{\mathcal{E}}}(\tau)(1+\mathfrak{r}(\tau, s))
$$

with $|\mathfrak{r}(\tau, s)| \leq \mathrm{e}^{-\varepsilon|s|}$ for some $\varepsilon=\varepsilon_{\mathcal{E}} \geq 0$. Here, $\lambda_{q_{\mathcal{E}}}$ is a positive constant. If $\mathcal{E} \subset C$ is a negative end, then (1.7) holds when $s \leq-s_{\mathcal{E}}$ with $\lambda_{q_{\mathcal{E}}}$ a negative constant. The integer $q_{\mathcal{E}}$ is said to be the multiplicity of the end $\mathcal{E}$. The function $\varsigma_{q_{E}}(\tau)$ can be assumed to be a $\left(2 \pi q_{\mathcal{E}}\right)$-periodic eigenfunction of a symmetric, $\mathbb{R}$-linear operator

$$
P_{\gamma}: C^{\infty}(\mathbb{R} ; \mathbb{C}) \rightarrow C^{\infty}(\mathbb{R} ; \mathbb{C}), \quad \eta \mapsto \frac{\mathrm{i}}{2} \frac{d}{d \tau} \eta+\nu \eta+\mu \bar{\eta} .
$$

Here, $\nu$ is a real-valued function with period $(2 \pi)$, and $\mu$ is a $\mathbb{C}$-valued periodic function with period $(2 \pi)$. They are determined by $f$ and $J$. The constant $\lambda_{\mathcal{E}}$ in (1.7) is the corresponding eigenvalue. With regard to terminology: A function, $\eta$, on $\mathbb{R}$ is said to be $(2 \pi q)$-periodic for some positive integer $q$ if $\eta(\cdot+2 \pi q)=\eta(\cdot)$. The period of $\eta$ is the least positive integer for which the above equality holds. The assumption that $\gamma$ is non-degenerate guarantees that the kernel of the operator $P_{\gamma}$ has no non-trivial elements with period $(2 \pi q)$ for any $q \in \mathbb{Z}^{+}$.

1.1.6. The differential. It follows from (1.7) that the image via the projection from $\mathbb{R} \times M$ to $M$ of a pseudoholomorphic subvariety $C$ defines a two-dimensional integer cycle, which we denote by $[C]$.

Fix elements $\Theta_{+}$and $\Theta_{-}$from the set $\mathcal{A}$ that gives the generators for periodic Floer homology. We define the set $\mathcal{M}_{1}\left(\Theta_{+}, \Theta_{-}\right)$as follows: If the relative $\mathbb{Z} / p \mathbb{Z}$-grading $I\left(\Theta_{+}, \Theta_{-}\right) \neq 1$, let $\mathcal{M}_{1}\left(\Theta_{+}, \Theta_{-}\right)=\emptyset$. Otherwise, if $I\left(\Theta_{+}, \Theta_{-}\right)=1 \bmod p$, let $\mathcal{M}_{1}\left(\Theta_{+}, \Theta_{-}\right)$be the set consisting of elements of the following form:

(1) An element $\Sigma \in \mathcal{M}_{1}\left(\Theta_{+}, \Theta_{-}\right)$is itself a finite set consisting of pairs the form $(C, m)$, where $C$ is an embedded, connected pseudoholomorphic submanifold in $\mathbb{R} \times M$, and $m$ is a positive integer. The integer $m=1$ unless $C=\mathbb{R} \times \gamma$, where $\gamma$ is an elliptic periodic orbit.

(2) If $(C, m),\left(C^{\prime}, m^{\prime}\right)$ are distinct pairs in $\Sigma$, then $C, C^{\prime}$ are disjoint.

(3) The weighted pseudoholomorphic curves constituting $\Sigma$ have the appropriate asymptotic behaviors and weights such that the cycle

$$
Z_{\Sigma}=\sum_{(C, m) \in \Sigma} m C
$$


defines an element in $H_{2}\left(M ; \Theta_{+}, \Theta_{-}\right)$. We call this element the relative homology class of $\Sigma$.

(4) Denote the above relative homology class by $\left[Z_{\Sigma}\right] \in H_{2}\left(M ; \Theta_{+}, \Theta_{-}\right)$. Then $\left[Z_{\Sigma}\right]$ must be among the relative homology classes satisfying

$$
I\left(\Theta_{+}, \Theta_{-},\left[Z_{\Sigma}\right]\right)=1 \in \mathbb{Z}
$$

The assumption that $\Gamma$ is monotone has the following important consequence: First, note that because of the property (1.5) of the relative index $I$, the set of relative homology classes meeting the constraint (4) above is a torsor over $\operatorname{Ker} c_{\Gamma}$, where $c_{\Gamma}$ is viewed as a homomorphism from $H_{2}(M ; \mathbb{Z})$ to $\mathbb{Z}$. The monotonicity of $\Gamma$ implies that $\left[w_{f}\right]$, also viewed as a homomorphism from $H_{2}(M ; \mathbb{Z})$ to $\mathbb{Z}$, restricts to a trivial map from $\operatorname{Ker} c_{\Gamma}$. That is to say, for every $\Sigma \in \mathcal{M}_{1}\left(\Theta_{+}, \Theta_{-}\right)$, the integral

$$
\int_{\Sigma} w_{f}=\sum_{\left(C_{i}, m_{i}\right) \in \Sigma} m_{i} \int_{C_{i}} w_{f}
$$

has the same value. This provides the type of "energy bound" needed for the typical Gromov compactness argument to establish the compactness of $\mathcal{M}_{1}\left(\Theta_{+}, \Theta_{-}\right)$(cf., e.g., Lemma 9.8 in $\left.[\mathbf{H 1}]\right)$.

With this understood, we shall define in Section 2.2 a residual subset $\mathcal{J}_{1 f} \subset \mathcal{J}_{f}$, with the following property: If $J \in \mathcal{J}_{1 f}$, then the set $\mathcal{M}_{1}\left(\Theta_{+}, \Theta_{-}\right)$ has the structure of a smooth, one-dimensional manifold with a finite set of components. Moreover, each component is a free orbit of the $\mathbb{R}$-action that is induced by the action of $\mathbb{R}$ on $\mathbb{R} \times M$ by constant translations along the $\mathbb{R}$ factor. As a parenthetical remark, note that an admissible almost complex structure need not be in $\mathcal{J}_{1 f}$.

Assume, in what follows, that $J$ is from the set $\mathcal{J}_{1 f}$.

Let $\mathfrak{o}_{+}, \mathfrak{o}_{-}$, respectively, denote a choice of the ordering of the elements in $\Theta_{+}$and $\Theta_{-}$whose periodic orbit component is positive hyperbolic. Given a $J \in \mathcal{J}_{1 f}$, each component of $\mathcal{M}_{1}\left(\Theta_{+}, \Theta_{-}\right)$can be assigned a weight from the set $\{ \pm 1\}$ once a choice of the orderings, $\mathfrak{o}_{+}, \mathfrak{o}_{-}$is made. This is described in Section 9 of [HT1], where similar weights are assigned to the pseudoholomorphic subvarieties that are used to construct the differential for embedded contact homology. Note that we are implicitly assuming here and in what follows that a product structure has been chosen for a certain natural $\mathbb{Z} / 2$ bundle over the set of all hyperbolic orbits of $\partial_{t}$. There is canonical isomorphism between the respective chain complexes that are defined by different product structures.

If $\mathcal{M}_{1}\left(\Theta_{+}, \Theta_{-}\right)$is not empty, use $\sigma\left(\Theta_{+}, \Theta_{-}\right) \in \mathbb{Z}$ to denote the sum of the \pm 1 weights associated to its components with respect to the choice of orderings $\mathfrak{o}$ made following the definition of $C P$ in (1.4). Set $\sigma\left(\Theta_{+}, \Theta_{-}\right)$ equal to 0 when $\mathcal{M}_{1}\left(\Theta_{+}, \Theta_{-}\right)$is empty. 
With the digression now over, we note that the differential for periodic Floer homology is defined by taking the linear extension of the following action on the generators:

$$
\partial \Theta_{+}=\sum_{\Theta_{-} \in \mathcal{A}} \sigma\left(\Theta_{+}, \Theta_{-}\right) \Theta_{-} .
$$

This endomorphism of $\mathbb{Z} \mathcal{A}$ has square zero; a proof can be had by taking almost verbatim the proof in [HT2] that the corresponding embedded contact homology differential has square zero. The endomorphism $\partial$ also decreases the $\mathbb{Z} / p \mathbb{Z}$ degree by 1 .

In summary, we have defined a Floer chain complex with coefficient $\mathbb{Z}$ to each parameter set:

$$
\boldsymbol{\mu}_{P}=\left\{\left(F, w_{F}\right), f, \Gamma, J\right\},
$$

where $\left(F, w_{F}\right)$ is an oriented closed surface with volume form $w_{F}, f$ is a nondegenerate volume-preserving automorphism of $F, \Gamma$ is a monotone class in $H_{1}(M ; \mathbb{Z})$ with respective to $w_{f}$, and $J \in \mathcal{J}_{1 f}$ is an almost complex structure on $\mathbb{R} \times M$. The resulting $\mathbb{Z} / p \mathbb{Z}$-graded homology is by definition the periodic Floer homology associated to $\boldsymbol{\mu}_{P}$, which we denote by

$$
H P_{*}\left(f:\left(F, w_{F}\right) \circlearrowleft, \Gamma\right)_{J}=H\left(C P_{*}, \partial\right),
$$

or simply $H P_{*}(f, \Gamma)$ when there is no danger of confusion.

1.2. Seiberg-Witten-Floer cohomology: the monotone case. Some stage setting is needed before describing the relevant version of the SeibergWitten-Floer cohomology. What follows is a brief description of how this cohomology is defined. The reader should look at Chapter 29 of $[\mathbf{K M}]$ for the detailed story.

1.2.1. Spin ${ }^{\mathbb{C}}$ structures. Given an orientated three-manifold $M$, a Spin ${ }^{\mathbb{C}}$ structure $\mathfrak{s}$ on $M$ is an equivalence class of a pair consisting of a Riemannian metric on $M$ and a lift, $\pi: \mathcal{F} \rightarrow M$, of the principle $\mathrm{SO}(3)$-bundle of oriented, orthonormal frames for $T M$ to a $U(2)$-principle bundle. Two such pairs are equivalent if there is a path of metrics and a corresponding lift along the path that interpolates between one pair and the other. The set of equivalence classes is a torsor modeled on $H^{2}(M ; \mathbb{Z})$.

Let $\mathbb{S}$ denote the associated bundle $\mathcal{F} \times_{U(2)} \mathbb{C}^{2}$. The standard Hermitian metric on $\mathbb{C}^{2}$ gives $\mathbb{S}$ a Hermitian fiber metric. The bundle $\mathbb{S}$ is a Clifford module for $T^{*} M$. This is to say that there is an endomorphism, cl: $T^{*} M \rightarrow$ $\operatorname{End}(\mathbb{S})$, such that

$$
\operatorname{cl}\left(a_{1}\right) \operatorname{cl}\left(a_{2}\right)=-\left\langle a_{1}, a_{2}\right\rangle-\operatorname{cl}\left(*\left(a_{1} \wedge a_{2}\right)\right),
$$

where $\langle\cdot, \cdot\rangle$ denotes the metric inner product. Endomorphisms in the image of $\mathrm{cl}$ are anti-Hermitian. The endomorphism cl induces two auxiliary homo- 
morphisms. The first, $\hat{\mathrm{c}}$, is defined as:

$$
\hat{c}: \mathbb{S} \otimes T^{*} M \rightarrow \mathbb{S}, \quad \eta \otimes b \mapsto \operatorname{cl}(b) \eta .
$$

The second map is a quadratic, bundle-preserving map from $\mathbb{S}$ to $\mathrm{i} T^{*} M$. Given $\eta \in \mathbb{S}$, we will write in what follows its image under this map as $\eta^{\dagger} \tau \eta$; the latter is in turn defined by the rule:

$$
\left\langle b, \eta^{\dagger} \tau \eta\right\rangle=\eta^{\dagger} \operatorname{cl}(b) \eta
$$

Let $\operatorname{det}(\mathbb{S})$ denote the complex, Hermitian line bundle $\bigwedge^{2} \mathbb{S}$. Its first Chern class, $c_{1}(\operatorname{det} \mathbb{S}) \in H^{2}(M ; \mathbb{Z})$, is called the canonical cohomology class of the Spin ${ }^{\mathbb{C}}$ structure $\mathfrak{s}$, and is often denoted as $c_{1}(\mathfrak{s})$. A Hermitian connection, $\mathbb{A}$, on $\operatorname{det}(\mathbb{S})$ and the Levi-Civita connection on $T M$ together define a metric compatible covariant derivative, $\nabla_{\mathbb{A}}$, on the space of sections of $\mathbb{S}$. This covariant derivative is used to construct the corresponding Dirac operator,

$$
D^{\mathbb{A}}=\hat{\mathrm{c}}\left(\nabla_{\mathbb{A}}\right): C^{\infty}(M ; \mathbb{S}) \rightarrow C^{\infty}(M ; \mathbb{S}) .
$$

In what follows, $B_{\mathbb{A}}$ denotes the Hodge star of the curvature two-form of the connection $\mathbb{A}$. This is an i $\mathbb{R}$-valued one-form on $M$.

1.2.2. The Seiberg-Witten equations. Fix an orientation for $M$, a Spin $^{\mathbb{C}}$ structure. Choose a metric on $M$ and a lift, $\pi: \mathcal{F} \rightarrow M$, of its oriented orthonormal frame bundle to a principle $U(2)$-bundle, so that the pair is in the equivalence class specified by the given $\operatorname{Spin}^{\mathbb{C}}$ structure. Let $\varpi$ be a closed two-form on $M$. We call a pair, $(\mathbb{A}, \Psi)$, consisting of a Hermitian connection on $\operatorname{det}(\mathbb{S})$ and a section of $\mathbb{S}$ a configuration. The group $C^{\infty}(M ; U(1))$ acts on the space of configurations in the following fashion: Let $u: M \rightarrow U(1)$. Then $u$ sends a configuration, $(\mathbb{A}, \Psi)$, to $\left(\mathbb{A}-2 u^{-1} d u, u \Psi\right)$. Two solutions obtained one from the other in this manner are said to be gauge equivalent. The group $C^{\infty}(M ; U(1))$ is called the gauge group.

In the most general form, the Seiberg-Witten equations ask that a configuration $(\mathbb{A}, \Psi)$ obey

$$
\left\{\begin{array}{l}
B_{\mathbb{A}}-\Psi^{\dagger} \tau \Psi+\mathrm{i} * \varpi-\mathfrak{T}=0 \quad \text { and } \\
D^{\mathbb{A}} \Psi-\mathfrak{S}=0,
\end{array}\right.
$$

where the pair $(\mathfrak{T}, \mathfrak{S})$ is a small perturbation arising as the formal gradient of a gauge-invariant function of $(\mathbb{A}, \Psi)$. It is in general needed to guarantee the transversality properties necessary for the definition of Seiberg-WittenFloer cohomology. See Chapters 10 and 11 in [KM].

Since the Seiberg-Witten equations are gauge invariant, the gauge group acts on the space of Seiberg-Witten solutions as well. Use $\mathcal{C}$, in what follows, to denote the set of gauge equivalence classes of solutions to (1.11). 
1.2.3. The cochain complex. Assume from this point on that:

The gauge group acts freely on the space of solutions to (1.11).

Then, for a suitably generic choice of $\varpi, \mathfrak{T}$, and $\mathfrak{S}$, the set $\mathcal{C}$ of gauge equivalence classes of solutions to (1.11) is finite. (In fact $(\mathfrak{T}, \mathfrak{S})$ can be set to be trivial for this purpose.)

Paraphrasing Definition 29.1.1 in $[\mathbf{K M}]$, the case when

$$
2 \pi c_{1}(\mathfrak{s})-[\varpi]=\mathrm{t} 2 \pi c_{1}(\mathfrak{s})
$$

when $\varpi \neq 0$ for some real $\mathrm{t}>0$ is said to be positive monotone, and it is said to be negative monotone when $\mathrm{t}<0$. The condition (1.12) holds in the monotone case (cf., e.g., Lemma 29.1.2 in $[\mathbf{K M}]$.)

Assume $\varpi \neq 0$ and monotonicity for the rest of this subsection. In this case, the cochain complex used to define the Seiberg-Witten-Floer cohomology is

$$
C M^{*}=\mathbb{Z} \mathcal{C}
$$

the free $\mathbb{Z}$-module that is generated by $\mathcal{C}$.

1.2.4. A relative grading. The complex $\mathbb{Z} \mathcal{C}$ has a relative $\mathbb{Z} / p \mathbb{Z}$-grading where $p$ is the divisibility in $H^{2}(M ; \mathbb{Z}) /$ Tors of the class $c_{1}(\mathfrak{s})$. This grading is defined using the spectral flow of a one-parameter family of unbounded, self-adjoint, Fredholm operators $\mathfrak{L}_{\mathfrak{c}}$ on $L^{2}\left(M ; \mathrm{i} T^{*} M \oplus \mathbb{S} \oplus \mathrm{i} \mathbb{R}\right)$, whose end members have trivial cokernel. The parameter $\mathfrak{c}$ of the family is from the space of configurations. The precise definition of $\mathfrak{L}_{\mathfrak{c}}$ will be given in (3.14); for now it suffices to say that the operator $\mathfrak{L}_{\mathfrak{c}}$ is obtained from the linearization of (1.11) at a given configuration $\mathfrak{c}=(\mathbb{A}, \Psi)$, and the operators associated to gauge equivalent configurations are conjugate to each other. A configuration $\mathfrak{c}$ is said to be non-degenerate when $\mathfrak{L}_{\mathfrak{c}}$ has trivial cokernel. As just noted, this notion only depends on the gauge equivalence class of $\mathfrak{c}$.

Let $\mathfrak{c}_{-}, \mathfrak{c}_{+}$be two non-degenerate gauge equivalence classes of configurations and use $\mathfrak{P}=\mathfrak{P}\left(\mathfrak{c}_{-}, \mathfrak{c}_{+}\right)$to denote the space of piecewise differentiable maps from $\mathbb{R}$ to the configuration space $\operatorname{Conn}(\operatorname{det} \mathbb{S})) \times C^{\infty}(M ; \mathbb{S})$ which have $s \rightarrow-\infty$ limit that is a configuration in the gauge equivalence class of $\mathfrak{c}_{-}$and $s \rightarrow \infty$ limit that is a configuration in the gauge equivalence class of $\mathfrak{c}_{+}$. The group $C^{\infty}(M, U(1))$ acts on $\mathfrak{P}$ by the following rule: an element $u \in C^{\infty}(M, U(1))$ sends a path $(\mathbb{A}(s), \Psi(s))$ to the path $\left(\mathbb{A}(s)-2 u^{-1} d u, u \Psi(s)\right)$. Two elements in $\mathfrak{P}$ in the same orbit under this action are said to be gauge equivalent.

The family of operators $\mathfrak{L}_{\mathfrak{c}(s)}$ associated to every element $\mathfrak{c}(s)$ in $\mathfrak{P}\left(\mathfrak{c}_{-}, \mathfrak{c}_{+}\right)$ has a well-defined spectral flow. It turns out that this spectral flow depends only on $\mathfrak{c}_{-}, \mathfrak{c}_{+}$, and the path component of the gauge equivalence class of $\mathfrak{c}(s)$ in the orbit space

$$
\mathcal{B}\left(\mathfrak{c}_{-}, \mathfrak{c}_{+}\right)=\mathfrak{P}\left(\mathfrak{c}_{-}, \mathfrak{c}_{+}\right) / C^{\infty}(M, U(1)) .
$$


With this understood, we call this class in $\pi_{0}\left(\mathcal{B}\left(\mathfrak{c}_{-}, \mathfrak{c}_{+}\right)\right)$the relative homotopy class of $\mathfrak{c}(s)$, and denote by $\mathcal{J}\left(\mathfrak{c}_{-}, \mathfrak{c}_{+} ; \mathfrak{h}\right) \in \mathbb{Z}$ the spectral flow of elements in $\mathfrak{P}\left(\mathfrak{c}_{-}, \mathfrak{c}_{+}\right)$with relative homotopy class $\mathfrak{h}$. Meanwhile, note that the set of relative homotopy classes, $\pi_{0}\left(\mathcal{B}\left(\mathfrak{c}_{-}, \mathfrak{c}_{+}\right)\right)$, is a torsor over the groups of components of the space of gauge transformations, i.e., $H^{1}(M ; \mathbb{Z}) \simeq H_{2}(M ; \mathbb{Z})$. Moreover, given two relative homotopy classes $\mathfrak{h}, \mathfrak{h}^{\prime}$ with their difference $\mathfrak{h}-\mathfrak{h}^{\prime}$ viewed as an element in $H_{2}(M ; \mathbb{Z})$, their respective spectral flows differ by

$$
\mathcal{J}\left(\mathfrak{c}_{-}, \mathfrak{c}_{+} ; \mathfrak{h}\right)-\mathcal{J}\left(\mathfrak{c}_{-}, \mathfrak{c}_{+} ; \mathfrak{h}^{\prime}\right)=\left\langle c_{1}(\mathfrak{s}), \mathfrak{h}-\mathfrak{h}^{\prime}\right\rangle .
$$

Finally, note that the genericity condition on $(\varpi, \mathfrak{T}, \mathfrak{S})$ is such that $\mathfrak{c}=$ $(\mathbb{A}, \Psi)$ is non-degenerate when it solves (1.11). Assume, in what follows, that the triple $(\varpi, \mathfrak{T}, \mathfrak{S})$ is chosen so that this condition hold. Applying the above discussion to the cases when $\mathfrak{c}_{-}, \mathfrak{c}_{+} \in \mathcal{C}$, it follows from (1.14) that the mod- $p$ reduction of the spectral flow,

$$
\mathcal{J}\left(\mathfrak{c}_{-}, \mathfrak{c}_{+}\right):=\mathcal{J}\left(\mathfrak{c}_{-}, \mathfrak{c}_{+} ; \mathfrak{h}\right) \bmod p,
$$

provides a relative $\mathbb{Z} / p \mathbb{Z}$-grading between any two generators $\mathfrak{c}_{-}, \mathfrak{c}_{+}$of the Seiberg-Witten-Floer cochain complex.

1.2.5. The differential. The differential for the Seiberg-Witten-Floer cohomology is defined using solutions to the Seiberg-Witten equations on $\mathbb{R} \times M$. These equations are viewed as a system of equations for a smooth $\operatorname{map} s \mapsto(\mathbb{A}(s), \Psi(s))$ from $\mathbb{R}$ to $\operatorname{Conn}(\operatorname{det}(\mathbb{S})) \times C^{\infty}(M ; \mathbb{S})$. The most general form of the equations read:

$$
\left\{\begin{array}{l}
\frac{d}{d s} \mathbb{A}+B_{\mathbb{A}}-\Psi^{\dagger} \tau \Psi+\mathrm{i} * \varpi-\mathfrak{T}=0, \\
\frac{d}{d s} \Psi+D^{\mathbb{A}} \Psi-\mathfrak{S}=0,
\end{array}\right.
$$

where $\varpi$ and $(\mathfrak{T}, \mathfrak{S})$ are as in (1.11). Given $\mathfrak{c}_{-}, \mathfrak{c}_{+} \in \mathcal{C}$, an instanton from $\mathfrak{c}_{-}$ to $\mathfrak{c}_{+}$is an element $(\mathbb{A}(s), \Psi(s))$ in $\mathfrak{P}\left(\mathfrak{c}_{-}, \mathfrak{c}_{+}\right)$solving the above equations. It is assigned a relative homotopy class and a spectral flow in the manner described in the previous paragraph. Moreover, a gauge transformation of an instanton is another instanton.

Let $\mathcal{M}_{1}\left(\mathfrak{c}_{-}, \mathfrak{c}_{+}\right)$denote the following: It is the empty set when $\mathcal{J}\left(\mathfrak{c}_{-}, \mathfrak{c}_{+}\right) \neq$ 1 . When $\mathcal{J}\left(\mathfrak{c}_{-}, \mathfrak{c}_{+}\right)=1 \bmod p$, let it be the space consisting of gauge equivalence classes of instantons from $\mathfrak{c}_{-}$to $\mathfrak{c}_{+}$with spectral flow 1. By (1.14), the relative homotopy classes of such instantons lie in a torsor over $\operatorname{Ker} c_{1}(\mathfrak{s})$. As explained in Section 30.1 of $[\mathbf{K M}]$, this fact, together with the monotonicity condition, ensures the compactness of $\mathcal{M}_{1}\left(\mathfrak{c}_{-}, \mathfrak{c}_{+}\right)$. With suitably generic choice of $(\varpi,(\mathfrak{T}, \mathfrak{S}))$, the space $\mathcal{M}_{1}\left(\mathfrak{c}_{-}, \mathfrak{c}_{+}\right)$will possess the following desirable properties: It is a one-dimensional smooth manifold diffeomorphic to a disjoint union of finitely many copies of $\mathbb{R}$, Moreover, the one-parameter 
group $\mathbb{R}$ acts freely on $\mathcal{M}_{1}\left(\mathfrak{c}_{-}, \mathfrak{c}_{+}\right)$by translating any instanton a constant amount with respect to the parameter $s \in \mathbb{R}$, and the aforementioned diffeomorphism from $\mathbb{R}$ to each component induces this $\mathbb{R}$-action. In addition, each one-dimensional component has an assigned weight, either +1 or -1 . Suffice it to say for now that this weight is obtained by comparing two canonical orientations. The first orientation is that defined by the generator of the $\mathbb{R}$-action; and the second is defined using the determinant line of a certain family of Fredholm operators that is obtained from the linearization of the expression on the left-hand side of (1.15). Use $\sigma\left(\mathfrak{c}_{-}, \mathfrak{c}_{+}\right)$to denote the sum of the \pm 1 weights that are assigned to the one-dimensional components of $\mathcal{M}_{1}\left(\mathfrak{c}_{-}, \mathfrak{c}_{+}\right)$when the set of such components is non-empty. Set $\sigma\left(\mathfrak{c}_{-}, \mathfrak{c}_{+}\right)$ to equal zero otherwise.

Given $\mathfrak{c} \in \mathcal{C}$, the action of the differential on $[\mathfrak{c}]$ is given by the following formula:

$$
\delta \mathfrak{c}_{+}=\sum_{\mathfrak{c}_{-} \in \mathcal{C}} \sigma\left(\mathfrak{c}_{-}, \mathfrak{c}_{+}\right) \mathfrak{c}_{-} .
$$

This differential has square zero and increases the relative $\mathbb{Z} / p \mathbb{Z}$-grading by 1 .

It turns out that in our setting, the perturbation terms $\mathfrak{T}$ and $\mathfrak{S}$ may be set as zero; and the assumption (1.12) and the various properties of $\mathcal{C}$ and $\mathcal{M}_{1}\left(\mathfrak{c}_{-}, \mathfrak{c}_{+}\right)$required for the definition of the Seiberg-Witten cohomology, as sketched above, may be obtained by a suitable generic choice of $\varpi$ alone.

1.2.6. The Seiberg-Witten cohomology. To summarize, in this subsection we defined a Floer cochain complex with coefficient $\mathbb{Z}$ associated to each parameter set:

$$
\boldsymbol{\mu}_{S}=\{M, \mathfrak{s}, \varpi, g, \mathfrak{q}\},
$$

where $M$ is an oriented closed three-manifold; $\mathfrak{s}$ and $\varpi$ are a Spin ${ }^{\mathbb{C}}$ structure and a closed two-form satisfying the monotonicity assumption (1.13); $g$ is a Riemannian metric on $M$; and $\mathfrak{q}=(\mathfrak{T}, \mathfrak{S})$ is as in (1.11). The Seiberg-Witten-Floer cohomology is defined to be the homology of the above Seiberg-Witten cochain complex, and it has a relative $\mathbb{Z} / p \mathbb{Z}$-grading. In the notation of Definition 30.1.1 in $[\mathbf{K M}]$, it is written as:

$$
H M^{*}(M, \mathfrak{s},-\pi[\varpi]):=H\left(C M^{*}, \delta\right) .
$$

It is explained in $[\mathbf{K M}]$ that the Seiberg-Witten-Floer cohomology depends only on the following triple:

- an oriented closed three-manifold $M$,

- a Spin ${ }^{\mathbb{C}}$-structure $\mathfrak{s}$ on $M$, and

- the non-trivial cohomology class

$$
p_{\mathfrak{s}, \varpi}:=2 \pi^{2} c_{1}(\mathfrak{s})-\pi[\varpi] \in H^{2}(M ; \mathbb{R}),
$$

modulo multiplication by positive numbers. 
Thus, the positive and negative monotone cases correspond to two different versions of Seiberg-Witten-Floer cohomologies. In particular, the positive monotone version agrees with the "ordinary Seiberg-Witten cohomology":

$$
H M^{*}(M, \mathfrak{s})=H M^{*}(M, \mathfrak{s}, 0) .
$$

Remarks. (1) The condition (1.12) implies that the $\widehat{H M}$ and $\widetilde{H M}$ versions of Seiberg-Witten cohomology defined in $[\mathbf{K M}]$ are the same, and the completed version $H M^{\bullet}$ is no different from the plain $H M^{*}$. Hence we denote all these by the same notation $H M^{*}$.

(2) In fact, to describe the dependence of the periodic Floer homology on $f$, it is more appropriate to regard $f$ as an element in $\widetilde{\operatorname{Symp}}\left(F, w_{F}\right)$, the universal covering of the symplectomorphism group of $\left(F, w_{F}\right)$. This will be clarified in Appendix 7.1 below.

1.3. The isomorphism theorem. We now describe a way to associate a Seiberg-Witten parameter set $\boldsymbol{\mu}_{S}$ to each Periodic Floer homology parameter set $\boldsymbol{\mu}_{M}$.

(1) From $f:\left(F, w_{F}\right) \circlearrowleft$ to $M$. Recall from the beginning of this section that a closed surface $F$ with volume form $w_{F}$ together with a volumepreserving map $f$ on it defines an associated mapping torus $M$ with orientation induced from $w_{F}$. They also define a closed two-form $w_{f}$ on $M$.

(2) From $f:\left(F, w_{F}\right) \circlearrowleft$ and $J$ to $g$. There is a standard way to define a metric $g$ on $M$ from $w_{F}$ and a $J \in \mathcal{J}_{f}$. See Section 3.3 below.

(3) From $\Gamma$ to $\mathfrak{s}$. There is an isomorphism between the space of $\mathrm{Spin}^{\mathbb{C}}$ structures and the homology group $H_{1}(M ; \mathbb{Z})$ as torsors over $H_{1}(M ; \mathbb{Z})$, as follows: Let $\mathfrak{s}$ be a $\operatorname{Spin}^{\mathbb{C}}$ structure on $M$ defined by the equivalence class of the Riemannian metric $g$ and a lifting $\mathcal{F} \rightarrow M$ of the frame bundle, as described in Section 1.2. Let $\mathbb{S}$ denote the associated $U(2)$-bundle of spinors. Clifford multiplication by the one-form $d t$ gives an anti-Hermitian automorphism of $\mathbb{S}$ whose square is a negative multiple of the identity. Write the corresponding orthogonal decomposition of $\mathbb{S}$ into eigenbundles as $E \oplus$ $E \otimes K^{-1}$ where $E \rightarrow M$ is a complex, Hermitian line bundle. The convention takes the left most summand to be that on which Clifford multiplication by $d t$ acts as a positive multiple of $i$. The assignment $\mathfrak{s} \mapsto c_{1}(\operatorname{det}(E))$ of a cohomology class to a Spin ${ }^{\mathbb{C}}$ structure defines a $1-1$ correspondence between the set of $\operatorname{Spin}^{\mathbb{C}}$ structures with $H^{2}(M ; \mathbb{Z})$, and equivalently, with $H_{1}(M ; \mathbb{Z})$ via Poincaré duality. Denote by $\mathfrak{s}_{\Gamma}$ the $\operatorname{Spin}^{\mathbb{C}}$ structure corresponding to the homology class $\Gamma \in H_{1}(M ; \mathbb{Z})$ under this $1-1$ correspondence, and by $\mathbb{S}_{\Gamma}$ the associated spinor bundle. Note that under this correspondence, the cohomology class $c_{\Gamma}$ in Section 1.1, which is relevant to the periodic Floer homology, equals the class $c_{1}\left(\mathbb{S}_{\Gamma}\right)$ in Section 1.2, which in turn is relevant to the Seiberg-Witten-Floer cohomology. In particular, this means that the 
two versions of $p$, defined differently in the afore-mentioned two subsections, are actually identical, thus justifying our use of the same notion for both.

(4) From $\Gamma$, wf to $\varpi$. Let

$$
\varpi_{r}=2 r w_{f}+\wp,
$$

where $r>0$ and $\wp$ is a closed two-form in the cohomology class $2 \pi c_{1}\left(\mathfrak{s}_{\Gamma}\right)$. With this choice,

$$
p_{\mathfrak{s}_{\Gamma}, \varpi_{r}}=-2 r \pi\left[w_{f}\right] .
$$

Note that with these choices, $\Gamma$ is positive or negative monotone with respect to $\left[w_{f}\right]$ precisely when $\mathfrak{s}_{\Gamma}$ and $\left[\varpi_{r}\right]$ are, respectively, positive or negative monotone with respect to each other.

(5) The non-local perturbation. Let $\mathfrak{q}$ be arbitrary.

Because the Seiberg-Witten-Floer cohomology only depends on (1.17), the Seiberg-Witten-Floer cohomology associated to the above choices does not depend on the extraneous data $r, \mathfrak{q}$, and the choice of $\wp$ within its cohomology class.

We are now ready to state the isomorphism theorem under the monotonicity assumption.

Theorem 1.1. Let $\boldsymbol{\mu}_{P}=\left\{\left(F, w_{F}\right), f, \Gamma, J\right\}$ be a periodic Floer parameter set described in (1.10), where $\Gamma \in H_{1}(M ; \mathbb{Z})$ is monotone with respect to $\left[w_{f}\right]$; and associate to it a Seiberg-Witten parameter set $\boldsymbol{\mu}_{S}=\left\{M, \mathfrak{s}_{\Gamma}, \varpi_{r}, g, \mathfrak{q}\right\}$ according to the recipe described above. Then there is an isomorphism between the two Floer (co)homologies with coefficient $\mathbb{Z}$ :

$$
H P_{*}\left(f:\left(F, w_{F}\right) \circlearrowleft, \Gamma\right)_{J} \simeq H M^{-*}\left(M, \mathfrak{s}_{\Gamma},-\pi\left[\varpi_{r}\right]\right),
$$

which reverses the relative $\mathbb{Z} / p \mathbb{Z}$-gradings. In particular, if the degree $d_{\Gamma}<$ 0 , then both Floer (co)homologies vanish. If $d_{\Gamma}=0$, then both sides are isomorphic to $\mathbb{Z}$.

Since $\left[w_{f}\right]$ is invariant under Hamiltonian isotopies of $f$, and since the Seiberg-Witten-Floer cohomology on the right-hand side of the isomorphism is invariant under rescaling of $\left[w_{f}\right]$ by positive numbers, an immediate consequence of the above isomorphism is:

Corollary 1.1. The periodic Floer homology only depends on the orientation of $F$, the Hamiltonian isotopy class of $f$, and the monotone homology class $\Gamma$.

By working with local coefficients, both the periodic Floer homology and the Seiberg-Witten-Floer cohomology have a more general definition, where $\Gamma$ need not be monotone. The above isomorphism theorem and corollary have corresponding generalizations, see Theorem 6.2 and Corollary 6.1 below. 
Remarks. (1) The assertions of Theorem 1.1 when $d_{\Gamma} \leq 0$ follow from Theorem 1.3 in the article $S W \Rightarrow G r$ from [T6]. See also the main theorem in $[\mathbf{K T}]$.

(2) The equality between the Euler characteristics of the two Floer (co)homologies in Theorems 1.1 and 6.2 follows from Theorem 1 in the article $S W=G r$ from [T6]; see also [MT, HL].

(3) This isomorphism theorem is related to a conjecture of D. Salamon, see Conjecture 10.1 in $[\mathbf{S}]$. Roughly speaking, this conjecture says that the Seiberg-Witten cohomology of the mapping torus of $f$ is equivalent to a certain version of the Floer homology of symplectic fixed points for certain self map of $d$-fold symmetric product of $F$ that is defined from $f$. By viewing the elements $\left\{\left(C_{i}, m_{i}\right)\right\}_{i}$ in $\mathcal{M}_{1}\left(\Theta_{+}, \Theta_{-}\right)$as holomorphic (branched) multisections of the surface bundle $\mathbb{R} \times M \rightarrow \mathbb{R} \times S^{1}$, the periodic Floer homology corresponds intuitively to this latter Floer homology.

As we noted before, when $\Gamma$ is positive monotone or negative monotone, the Seiberg-Witten cohomology on the right-hand side of the isomorphism corresponds, respectively, to the positive monotone or negative monotone version described in Section 30.1 of $[\mathbf{K M}]$; in particular, in the former case, it coincides with the "ordinary Seiberg-Witten cohomology" $H M^{-*}\left(M, \mathfrak{s}_{\Gamma}\right)$. On the other hand, Taubes proved a sister version of the above theorem in [T1-T4], which states that

Theorem. Given a closed contact three-manifold $M$, and a homology class $\Gamma \in H_{1}(M ; \mathbb{Z})$, there is an isomorphism from the associated embedded contact homology ECH $H_{*}$ to the Seiberg-Witten-Floer cohomology $\widehat{H M}^{-*}\left(M, \mathfrak{s}_{\Gamma}\right)$ which reverses the relative gradings.

In the above, $\mathfrak{s}_{\Gamma}$ is defined in the same way as in item (3) in the beginning of this subsection, with $c_{1}\left(K^{-1}\right)$ now denoting the Euler class of the twoplane field which defines the contact structure.

Recall from the last remark in Section 1.2 that

$$
\widehat{H M}^{-*}(M, \mathfrak{s})=H M^{-*}(M, \mathfrak{s})
$$

when $c_{1}(\mathfrak{s})$ is non-torsion; it follows immediately that

Corollary 1.2. Let $M$ be the mapping torus of an orientation-preserving map $f:\left(F, w_{F}\right) \circlearrowleft$, and $\Gamma \in H_{1}(M ; \mathbb{Z})$ be a positive monotone class with respect to $\left[w_{f}\right]$, then

$$
E C H_{*}(M, \xi ; \Gamma) \simeq H P_{*}\left(f, \Gamma+h_{\xi}\right),
$$

where the left-hand side denotes the embedded contact homology of $(M, \xi)$; $\xi$ is a contact structure consistent with the orientation of $M$, and $h_{\xi}$ is Poincare dual to the primary obstruction to finding a homotopy between the two-plane fields $\xi$ and $\operatorname{Ker} \pi_{*}$. 
The isomorphism in Theorem 1.1 may be used to compute one Floer (co)homology from the other, depending on which is easier to compute in the context. Here are some sample applications of this type.

First, note that monotone classes with respect to a fixed $\left[w_{f}\right]$ are specified by their degree $d_{\Gamma}$. In particular, $d_{\Gamma}<g-1$ when $\Gamma$ is positive monotone, and $d_{\Gamma}>g-1$ when $\Gamma$ is negative monotone. By the definition of periodic Floer homology, $d_{\Gamma} \neq g-1$.

I. From HP to HM: The degree-1 periodic Floer homology of the mapping torus of $f$ is identical with a certain version of the symplectic Floer homology of $f$. (see Appendix 7.2 for a precise statement). The latter has been computed for all area-preserving surface automorphisms, see $[\mathbf{C}]$ and references therein. This implies that the corresponding Seiberg-WittenFloer cohomology of degree $<2$ is known for all mapping tori of areapreserving surface automorphisms as well. In [HS], the periodic Floer homology of certain Dehn twists has been computed. This also provides some new computations of the Seiberg-Witten-Floer cohomology.

II. From HM to HP: Corollary 31.5.2 in [KM] gives a long exact sequence relating the positive monotone version of $H M_{*}$, the negative monotone version of $H M_{*}$, and the bar-version of the Seiberg-Witten homology at the "balanced perturbation" $c_{b}=-2 \pi^{2} c_{1}(\mathfrak{s})$ (cf. Section 31.1 of $[\mathbf{K M}]$ ). The dual version of this exact sequence takes the following form:

$$
\begin{aligned}
\cdots & \stackrel{j^{*}}{\longrightarrow} H M^{*}\left(M, \mathfrak{s}, c_{+}\right) \stackrel{i^{*}}{\longrightarrow} \overline{H M}^{*}\left(M, \mathfrak{s}, c_{b}\right) \stackrel{p^{*}}{\longrightarrow} H M^{*}\left(M, \mathfrak{s}, c_{-}\right) \\
& \stackrel{j^{*}}{\longrightarrow} H M^{*}\left(M, \mathfrak{s}, c_{+}\right) \stackrel{i^{*}}{\longrightarrow} \cdots,
\end{aligned}
$$

where the first term and the third term above denote the positive monotone version and the negative monotone version of the Seiberg-Witten-Floer cohomology, respectively. Thus, knowledge of the ordinary Seiberg-Witten cohomology enables one to compute the periodic Floer homology in degrees larger than $g-1$.

Corollary 1.3 (Cotton-Clay). Suppose the genus $g$ of the surface $F$ is positive. Let $\Gamma$ be a monotone class with respect to $\left[w_{f}\right]$ with degree $d_{\Gamma}>$ $2 g-2$. Then

$$
H P_{*}(f, \Gamma) \simeq \overline{H M}^{-*}\left(M, \mathfrak{s}_{\Gamma}, c_{b}\right),
$$

where $\overline{H M}^{*}\left(M, \mathfrak{s}, c_{b}\right)$ denotes the bar-version of the Seiberg-Witten-Floer cohomology at the "balanced perturbation".

Proof. Let $\Gamma$ be a monotone class with degree $d_{\Gamma}>2 g-2 \geq g-1$ as in the statement of the corollary, and take $\mathfrak{s}=\mathfrak{s}_{\Gamma}$ in the above long exact sequence. By the equivalence $H M^{*}\left(M, \mathfrak{s}, c_{+}\right) \simeq H M^{*}(M, \mathfrak{s})$ (see Theorem 31.1.2 in $[\mathbf{K M}]$ ) and the adjunction inequality (see Corollary 40.1.2 in $[\mathbf{K M}]$, or rather, its dual version), this condition on degree implies that $H M^{*}\left(M, \mathfrak{s}, c_{+}\right)=0$. On the other hand, by Theorem 1.1, $H M^{*}\left(M, \mathfrak{s}, c_{-}\right) \simeq$ 
$H P_{-*}(f, \Gamma)$, since $\Gamma$ is negative monotone. The claim of the corollary follows by combining these observations with the long exact sequence above.

Remark. The bar-version of Seiberg-Witten-Floer cohomology is defined solely from the space of flat connections on $M$ and the family of Dirac operators parametrized by it. In Chapters 33-35 of $[\mathbf{K M}]$, Kronheimer and Mrowka showed that the bar-version of Seiberg-Witten-Floer homology can be computed from a "Coupled Morse chain complex" on the torus of flat connections. Given a Morse function on the torus $H^{1}(M ; \mathbb{R}) / H^{1}(M ; \mathbb{Z})$, this chain complex is constructed from its moduli spaces of gradient flow lines of dimensions 1 and 3, together with appropriately chosen Cech cocycles in cohomology classes determined by $c_{1}(\mathfrak{s})$ and the triple cup product $\Upsilon: \bigwedge^{3} H^{1}(M) \rightarrow H^{3}(M) \simeq \mathbb{Z}$. Explicit formulas are given in Chapter 35 of $[\mathbf{K M}]$ for $\overline{H M}_{*}$ in the special cases when $b_{1}(M) \leq 3$, or when $f$ is isotopic to the identity and $c_{1}(\mathfrak{s})$ is proportional to the Poincaré dual of $[F]$. A modification of the computation for the last example in $[\mathbf{K M}]$ finds that under the assumptions that $\left|\left\langle c_{1}(\mathfrak{s}),[F]\right\rangle\right|>0$ and $g>0$, the homology groups of these complexes are always non-torsion over $\mathbb{Z}$. Note in contrast that when $b_{1}(M)>1$, the Euler characteristics of both Seiberg-Witten-Floer cohomology and periodic Floer homology are trivial by the adjunction inequality for Seiberg-Witten invariants of three-manifolds, as the latter is independent of perturbations.

The previous corollary may be generalized as follows: By combining the isomorphism Theorem 1.1 with the involution in Seiberg-Witten theory, described in the Floer-homology context in Proposition 25.5.5 of [KM], one has the following:

If $\Gamma$ is a monotone class of degree $d>g-1$ with respect to a certain $\left[w_{f}\right]$, then $H P_{*}(f, \Gamma) \simeq H M^{-*}\left(M, \mathfrak{s}_{\Gamma^{*}}, c_{-}\right)$, where $\Gamma^{*}$ is the monotone class of degree $2 g-2-d$ with respect to the same class $\left[w_{f}\right]$. Thus, it can be computed from $\overline{H M}^{*}\left(M, \mathfrak{s}_{\Gamma^{*}}, c_{b}\right)$ and $H M^{*}\left(M, \mathfrak{s}_{\Gamma^{*}}, c_{+}\right)$by the long exact sequence (1.18). This means that the periodic Floer homology is completely determined by the ordinary and bar-versions of the Seiberg-Witten-Floer cohomologies for monotone classes of degrees $0<d<g-1$, and the $i^{*}$-maps between the two versions of $H M^{*}$. In particular, it implies that the periodic Floer homology with coefficient $\mathbb{Z}$ for all surfaces of $g \leq 2$, when it is welldefined, is completely determined by the cohomology of $M$, except the case when $g=2$ and $e_{\Gamma}=c_{1}(K)$. In this last case it is determined by the $i^{*}$-map from the relevant ordinary Seiberg-Witten-Floer cohomology, which is $\mathbb{Z}$ in this case.

1.3.1. Proving the isomorphism theorems. The isomorphism stated in Theorems 1.1 and 6.2 is obtained by using a version of Seiberg-Witten equations (1.11), parametrized by $r \in \mathbb{R}^{+}$, to define the cochain complex for the 
Seiberg-Witten-Floer cohomology. For suitably chosen $f$ and $J$ (cf. Section 2) and any sufficiently large $r$, we construct an isomorphism between the periodic Floer homology complex and the corresponding Seiberg-WittenFloer cohomology complex, which reverses the relative $\mathbb{Z} / p \mathbb{Z}$-grading. Note in contrast that there are no such isomorphisms between the $E C H$ and $H M$ complexes in the proof of $E C H=H M$ in $[\mathbf{T 1}, \mathbf{T 2}, \mathbf{T 3}, \mathbf{T} 4]$. Instead, the latter proof relies on a filtration on the Floer (co)chain complexes, and constructed injective chain maps from finitely generated sub-complexes of the $E C H$ chain complex to the $H M$ cochain complex. An isomorphism between the respective Floer (co)-homologies is obtained after taking a direct limit. This filtration argument is not needed in this article; this is because the appearance of the non-exact perturbation two-form $w_{f}$ guarantees that the Seiberg-Witten-Floer cochan complex in our context is finitely generated.

The proofs of Theorems 1.1 and 6.2 have the same analytic details, which are in most respects very similar to those in the proof of Theorem 1 in [T1], which is in turn based on the techniques from [T6]. In particular, most of the proof of Theorem 1 can be borrowed with only notational or cosmetic changes from corresponding arguments in $[\mathbf{T 1}, \mathbf{T 2}, \mathbf{T 3}, \mathbf{T 4}]$. This said, the authors have chosen to direct the reader to such arguments when appropriate with faith that the reader can make the required changes. The detailed arguments in what follows give those parts of the proof of Theorems 1.1 and 6.2 that differ substantially from their $[\mathbf{T 1}, \mathbf{T 2}, \mathbf{T 3}, \mathbf{T 4}]$ analog. Note in this regard that to show that the map from $E C H$ to $H M$ is onto, the arguments in $[\mathbf{T 1}, \mathbf{T} 4]$ use at key points an estimate for the spectral flow of a certain one-parameter family of Dirac operators. This is the spectral flow estimate given by Proposition 5.1 of [T7]. This spectral flow estimate plays a profound role in $[\mathbf{T 1}, \mathbf{T 4}]$ and is the key new idea which was not present in [T6]. As it turns out, there is no need here for this spectral flow estimate; judicious use of the action functional in Seiberg-Witten-Floer theory and cohomological considerations of the sort used in the article $S W \Rightarrow G r$ from [T6] suffice. (For example, none of Section 5 in [T4] is needed here.) Similar arguments are used in [L1] to prove an analog of $S W \Rightarrow G r$ in the more general setting of four-manifold with cylindrical ends equipped with an asymptotically constant harmonic two-form, which may not be nowhere vanishing, and where higher-dimensional moduli spaces are also considered.

The proof of Theorem 1.1 is given in Section 4.4, and Section 6.3 contains the modification needed to generalize to Theorem 6.2.

1.4. Notation and conventions. What follows uses $c_{0}$ to denote a constant that is greater than 1 . Unless a specific note to the contrary is present, this constant is independent of other relevant parameters in the discussion. The precise value of $c_{0}$ can be assumed to increase in subsequent appearances. The letter $\kappa$ is used for a similar purpose. 
The constructions in the subsequent sections use a fixed, non-increasing function, $\chi:[0, \infty) \rightarrow[0,1]$ which takes value 1 on $\left[0, \frac{5}{16}\right]$ and is zero on $\left[\frac{7}{16}, \infty\right)$.

On the Seiberg-Witten-Floer theory side, we follow the gauge-theory literature in which the boundary maps are defined by flows from the $s \rightarrow-\infty$ side to the $s \rightarrow \infty$ side (and therefore the coboundary maps go in the opposite direction).

On the periodic Floer homology side, we follow the symplectic Floer theory literature in which the boundary maps are defined by flows from the $s \rightarrow \infty$ side to the $s \rightarrow-\infty$ side.

This accounts for our notational convention that in the various corresponding notations in the two Floer theories, the positive and the negative ends appear in the opposite order, such as $\mathcal{M}_{1}\left(\Theta_{+}, \Theta_{-}\right)$versus $\mathcal{M}_{1}\left(\mathfrak{c}_{-}, \mathfrak{c}_{+}\right)$. It is for the same reason that the periodic Floer homology is isomorphic to the Seiberg-Witten-Floer cohomology.

\section{Deforming the map $f$ and the almost complex structure $J$}

The proof of Theorem 1.1 requires two preliminary steps. These steps are needed in order to use the techniques from $[\mathbf{T 1}, \mathbf{T 2}, \mathbf{T 3}, \mathbf{T} 4]$. The first step makes a small modification to the diffeomorphism, $f$, that defines $M$ and the associated periodic Floer homology chain complex. The second step makes a small modification to the almost complex structure on $\mathbb{R} \times M$ that is used to construct the differential on this same chain complex. With a class $\Gamma \in H_{1}(M ; \mathbb{Z})$ given, the deformations that are described below can be made so the original and the new periodic Floer homology chain complexes have the same set of generators and identical differentials. The deformation depends on the choice of a pair $(\delta, N)$, where $\delta$ is a small, positive number, and $N$ is a positive integer.

Let $\Lambda_{N}$ denote the set of periodic orbits of $f$ with period $N$ or less. If $\Gamma \in H_{1}(M ; \mathbb{Z})$ is any given class, then there exists $N$ such that $\Gamma$ 's version of the set $\mathcal{A}$ is defined solely from periodic orbits in $\Lambda_{N}$.

2.1. The deformation of $f$. A small deformation of $f$ is described here, It changes $f$ only near the points that comprise the periodic orbits in $\Lambda_{N}$ but fixes these same orbits.

Fix $\gamma \in \Lambda_{N}$ and let $q \in\{1, \ldots, N\}$ denote its period. A pair of functions $z=\left(z_{1}, z_{2}\right)$ defined on a neighborhood of $\mathrm{p} \in F$ are said to define a symplectic coordinate chart centered at $\mathrm{p}$ when they vanish at $\mathrm{p}$ and are such that $w_{F}$ is given by $d z_{1} \wedge d z_{2}$ near p. Given such a pair of function $z=\left(z_{1}, z_{2}\right)$, then $f^{q}$ appears as a map that can be written as

$$
z \mapsto K_{\gamma} \cdot z+\mathfrak{q}(z), \quad \text { where }|\mathfrak{q}| \leq c_{0}|z|^{2} \text { and where } K_{\gamma} \in \operatorname{Sl}(2 ; \mathbb{R}) .
$$


If $\gamma$ is elliptic, then the matrix $K_{\gamma}$ is conjugate in $\mathrm{Sl}(2 ; \mathbb{R})$ to a rotation by an angle $2 \pi \mathrm{R}$, where $\mathrm{R} \in(0,1)$. This $\mathrm{R}$ is called the rotation number of the periodic orbit. It does not depend on the chosen point $\mathrm{p} \in \gamma$ or on the chosen symplectic coordinate chart. Moreover, if $\gamma$ is non-degenerate, $\mathrm{R}$ is irrational. If $\gamma$ is hyperbolic, then $K_{\gamma}$ is conjugate in $\mathrm{Sl}(2 ; \mathbb{R})$ to a matrix of the form

$$
\left[\begin{array}{cc}
\lambda & 0 \\
0 & \lambda^{-1}
\end{array}\right]
$$

where $\lambda$ is a real number with $|\lambda|>1$. In all cases, the eigenvalues of $K_{\gamma}$ do not depend on the choice of the symplectic coordinate chart.

Definition 2.1. Given $K_{\gamma}$, define functions $\nu_{\gamma}$ and $\mu_{\gamma}$ on $[0,2 \pi]$ from the type and rotation number of $\gamma$ as follows:

- If $\gamma$ is elliptic with rotation number $\mathrm{R}$, then $\nu_{\gamma}=\frac{1}{2} \mathrm{R}$ and $\mu_{\gamma}=0$.

- If $\gamma$ is hyperbolic, then $\nu_{\gamma}=\frac{1}{2} \mathrm{R}$ and $\mu_{\gamma}=-\frac{\mathrm{i}}{4 \pi} \ln |\lambda| \mathrm{e}^{2 \mathrm{iR} \tau}$ with

$$
\mathrm{R}= \begin{cases}0 & \text { when } \gamma \text { is positive hyperbolic, } \\ \frac{1}{2} & \text { when } \gamma \text { is negative hyperbolic, }\end{cases}
$$

where $\lambda$ is as in (2.2).

Fix $z \in \mathbb{C}$, and solve the equation

$$
\frac{\mathrm{i}}{2} \partial_{\tau} \eta+\nu_{\gamma} \eta+\mu_{\gamma} \bar{\eta}=0
$$

for a function $\eta: \mathbb{R} \rightarrow \mathbb{C}$ with $\eta(0)=z$. With $\eta$ and $z$ viewed as vectors in $\mathbb{R}^{2}$, the solution has the form $\eta(\tau)=U(\tau) \cdot z$, where $U$ is a map from $\mathbb{R}$ to $\mathrm{Sl}(2 ; \mathbb{R})$ with the following properties:

- $U(0)$ is the identity.

- If $\gamma$ is elliptic with rotation number $\mathrm{R}$, then $U(2 \pi)$ is the rotation through angle $2 \pi \mathrm{R}$.

- If $\gamma$ is hyperbolic, then $U(2 \pi)$ is the matrix in (2.2).

For $k \in \mathbb{Z}$, set

$$
U_{\gamma}^{k / q}=U(2 \pi k / q)
$$

Fix a point $\mathrm{p} \in \gamma$. As the almost complex structure $J$ on $\mathbb{R} \times M$ must map $\operatorname{Ker}\left(\pi_{*}\right)$ to itself along $\mathbb{R} \times \gamma$; thus it defines an almost complex structure on $\left.T F\right|_{\mathrm{p}}$. The latter is denoted by $\mathfrak{j}_{p}$. Since $\mathfrak{j}_{p}^{2}=-1$, it follows that there exist symplectic coordinate chart $z=\left(z_{1}, z_{2}\right)$ for $F$ near $\mathrm{p}$, such that $\mathfrak{j}_{p} \frac{\partial}{\partial z_{1}}=\frac{\partial}{\partial z_{2}}$. Coordinates of this sort are said to be $J$-adapted.

What follows describes the desired modification of $f$.

Lemma 2.1. Fix a positive integer $N$. Then there exists a constant $\kappa \geq 1$ such that given $\delta<\kappa^{-1}$, there exists a diffeomorphism $f^{\prime}: F \rightarrow F$ with the following properties: 
(1) The map $f^{\prime}$ is area-preserving, which is to say that $\left(f^{\prime}\right)^{*} w_{F}=w_{F}$.

(2) All periodic orbits of $f^{\prime}$ are non-degenerate.

(3) The maps $f$ and $f^{\prime}$ have the same set of periodic orbits with period $N$ or less.

Moreover, corresponding orbits are either both elliptic or both hyperbolic. If both elliptic, then they have the same rotation number. If both hyperbolic, then they are either both positive hyperbolic or negative hyperbolic. In either case, the eigenvalues of the corresponding versions of the matrix $K_{(\cdot)}$ agree.

(4) Let $\gamma$ denote a periodic orbit of period $q \leq N$. Then there are $J$ adapted, symplectic coordinates about the points that comprise $\gamma$ such that for each $k \in\{0, \ldots, q-1\}$ the map $f^{\prime}$ near $f^{k}(\mathrm{p})$ in these coordinates is given by

$$
z \mapsto U_{\gamma}^{(k+1) / q} \cdot\left(U_{\gamma}^{k / q}\right)^{-1} z+\mathfrak{p}_{k}(z),
$$

where $\mathfrak{p}_{k}$ is zero in a neighborhood of $z=0$.

(5) The coordinate disks in $F$ of radius $\delta$ about the points in the orbits from $\Lambda_{N}$ are $J$-adapted, pairwise disjoint; and the mapsf andf agree on the complement of their union.

This lemma is proved momentarily.

2.2. The modification of $\mathbf{J}$. A deformation of $f$ to a map $f^{\prime}$ as described in Lemma 2.1 is exploited in later sections with the help of a corresponding modification of the almost complex structure $J$. The definition of this modified almost complex structure requires a preliminary modification of the vector field $\partial_{t}$, so as to obtain a vector field whose return map gives $f$ near the points in $\Lambda_{N}$. The latter vector field is denoted by $\partial_{t}$, and it is constructed in Part 1 of this subsection. Part 4 describes an allowed set of almost complex structures that can serve as the desired modification of $J$. The intervening Parts 2 and 3 of this subsection jointly define one of the criteria that are used in Part 4 to describe the allowed set of modified almost complex structures.

Part 1. The embedding $\varphi_{\gamma}$ and the modified vector field $\partial_{t}^{\prime}$. It is assumed, in what follows, that $(\delta, N)$ has been chosen subject to the conditions in Lemma 2.1. Let $f^{\prime}$ denote a map given by (2.1) using this data. This modification of $f$ is used in what follows to construct a corresponding modification of the vector field $\partial_{t}$. The new version of $\partial_{t}$ is described below.

It proves useful to view elements in $\Lambda_{N}$ now as integral curves of $\partial_{t}$. Fix $\gamma \in \Lambda_{N}$, now viewed as a closed integral curve of the vector field $\partial_{t}$ on $M$. As such, $\gamma$ has a tubular neighborhood embedding $\varphi_{\gamma}: S^{1} \times D \rightarrow M$, where $D \subset \mathbb{C}$ is a disk of small radius. Such an embedding is described momentarily with certain desirable properties. 
Use $\tau$ to denote the affine parameter on $S^{1}$, and use $z$ to denote the complex coordinate for $\mathbb{C}$. The desired embedding, $\varphi_{\gamma}$, has the following properties:

Lemma 2.2. Given $N \geq 1$, there exists $\kappa \geq 1$ with the following significance:

Fix $\delta \in\left(0, \kappa^{-1}\right)$ and invoke Lemma 2.1 to construct a map $f^{\prime}$. Fix such an $f^{\prime}$, and let $D \subset \mathbb{C}$ denote the disk of radius $100 \delta$. Then there is a set $\left\{\varphi_{\gamma}: S^{1} \times D \rightarrow M\right\}_{\gamma \in \Lambda_{N}}$ of embeddings with pairwise disjoint images, and such that for each $\gamma \in \Lambda_{N}$, the embedding $\varphi_{\gamma}$ obeys:

- The composition of $\varphi_{\gamma}$ with the projection $\pi: M \rightarrow S^{1} \operatorname{sends}(\tau, z)$ to $q \tau$ with $q$ here denoting the period of $\gamma$.

- The vector field $q^{-1} \partial_{t}$ is the push-forward from $S^{1} \times D$ of a vector field that has the form

$$
\partial_{\tau}-2 \mathrm{i}(\nu z+\mu \bar{z}+\mathfrak{r}) \partial_{z}+2 \mathrm{i}(\nu \bar{z}+\bar{\mu} z+\overline{\mathfrak{r}}) \partial_{\bar{z}},
$$

where $\nu$ is an $\mathbb{R}$-valued function on $S^{1}$ and $\mu$ is a $\mathbb{C}$-valued function. Meanwhile $\mathfrak{r}$ is such that its absolute value is bounded by $\kappa|z|^{2}$ and its first derivatives are bounded in absolute value by $\kappa|z|$.

- Use $\varphi_{\gamma}$ to identify the disks $\bigcup_{0 \leq k \leq q}(\{2 \pi k / q\} \times D)$ with their embedded images via $\varphi_{\gamma}$ in $F=\pi^{-1}(0)$. Granted this identification, the map $f^{\prime}$ is obtained by traveling for time $2 \pi / q$ along the integral curves of the vector field

$$
\mathrm{v}_{\gamma}:=\partial_{\tau}-2 \mathrm{i}\left(\nu_{\gamma} z+\mu_{\gamma} \bar{z}+\mathfrak{r}^{\prime}\right) \partial_{z}+2 \mathrm{i}\left(\nu_{\gamma} \bar{z}+\bar{\mu}_{\gamma} z+\overline{\mathfrak{r}}^{\prime}\right) \partial_{\bar{z}},
$$

where $\mathfrak{r}^{\prime}$ has the following properties: It vanishes where $|z|$ is in a neighborhood of 0 , and it is equal to $\mathfrak{r}$ where $|z|>\delta$.

- The vector field $\varphi_{\gamma *} \partial_{z}$ along $\mathbb{R} \times \gamma$ is such that $J\left(\varphi_{\gamma *} \partial_{z}\right)=\varphi_{\gamma *}\left(\mathrm{i} \partial_{z}\right)$.

This lemma is proved in Section 2.4.

Define a vector field, $\partial_{t^{\prime}}$, on $M$ as follows:

$$
\partial_{t^{\prime}}= \begin{cases}\left(\varphi_{\gamma}\right)_{*} \mathrm{v}_{\gamma} & \text { over the image of } \varphi_{\gamma}, \text { for all } \gamma \in \Lambda_{N}, \\ \partial_{t} & \text { otherwise. }\end{cases}
$$

This vector field $\partial_{t^{\prime}}$ is the desired modification of $\partial_{t}$.

Part 2. The set of almost complex structures $\mathcal{J}_{0 f}$. In this and the next part, we digress to describe the criteria for membership in the subset $\mathcal{J}_{1 f}$ of the set of almost complex structures on $\mathbb{R} \times M$. The criteria are of two sorts. The first sort is a standard genericity condition: It requires that all irreducible, pseudoholomorphic subvarieties be non-degenerate in the sense that a certain associated Fredholm operator has trivial cokernel. To say more, suppose that $C \subset \mathbb{R} \times M$ is an irreducible, pseudoholomorphic subvariety. There 
exists a model curve for $C$, denoted $C_{0}$. This is a smooth, complex curve with a smooth, pseudoholomorphic map

$$
\varphi_{0}: C_{0} \rightarrow M
$$

which is $1-1$ on the complement of a finite set, onto $C$. The subvariety $C$ is said to be immersed when $\varphi_{0}$ is an immersion.

The operator in question has a relatively straightforward description in the case when $C$ is immersed. Supposing that such is the case, then a normal bundle $\mathrm{N} \rightarrow C_{0}$ is defined so that it restricts to any open set $U \subset C_{0}$ that is embedded by $\varphi_{0}$ as the pull-back via $\varphi_{0}$ of the normal bundle of $\varphi_{0}(U) \subset C$ in $\mathbb{R} \times M$. The almost complex structure $J$ endows $\mathrm{N}$ with the structure of a complex line bundle. This said, view $\mathrm{N}$ henceforth as a complex line bundle. Then there exists on $C_{0}$ a canonical, $\mathbb{R}$-linear Fredholm operator that maps sections over $C_{0}$ of $\mathrm{N}$ to those of $\mathrm{N} \otimes T^{0,1} C_{0}$. This operator is denoted as $\mathcal{D}_{C}$. Its action on a section $\eta$ of $\mathrm{N}$ can be written as

$$
\mathcal{D}_{C} \eta=\bar{\partial} \eta+\nu_{C} \eta+\mu_{C} \bar{\eta}
$$

where the notation is as follows: Fix a Hermitian metric on $\mathrm{N}$ so as to give the latter a holomorphic structure; this is used to define $\bar{\partial}$. Meanwhile, $\nu_{C}$ and $\mu_{C}$ are respective sections of $T^{0,1} C_{0}$ and $\mathrm{N}^{\otimes 2} \otimes T^{0,1} C_{0}$ that are defined using the one-jet along $C$ of $J$. Note that a change of Hermitian metric is compensated by a change in what is meant by $\nu_{C}$. This is why (2.4) is canonical. The operator $\mathcal{D}_{C}$ arises in the following way: Fix $\varepsilon>$ 0 and let $\eta$ denote a section of $\mathrm{N}$. The composition of first $\varepsilon \eta$ and then the metrics exponential map defines a deformation of the subvariety $C$. This deformation is pseudoholomorphic to first order in $\varepsilon$ if and only if $\mathcal{D}_{C} \eta=0$. The assumption that all periodic orbits are non-degenerate implies that $\mathcal{D}_{C}$ maps $L_{1}^{2}\left(C_{0} ; \mathrm{N}\right)$ to $L^{2}\left(C_{0} ; \mathrm{N} \otimes T^{0,1} C_{0}\right)$ as a Fredholm operator. The kernel of $\mathcal{D}_{C}$ is used here to denote the space of sections in $L_{1}^{2}\left(C_{0} ; \mathrm{N}\right)$ that are annihilated by $\mathcal{D}_{C}$. The cokernel of $\mathcal{D}_{C}$ is used here to denote its $L^{2}\left(C_{0} ; \mathrm{N}^{\otimes 2} \otimes T^{0,1} C_{0}\right)$ cokernel. This is the kernel of the formal adjoint. Note also that the kernel and cokernel elements are smooth. In any event, the index of $\mathcal{D}_{C}$ denotes its index as a Fredholm operator from $L_{1}^{2}\left(C_{0} ; \mathrm{N}\right)$ to $L^{2}\left(C_{0} ; \mathrm{N} \otimes T^{0,1} C_{0}\right)$. This Fredholm version of $\mathcal{D}_{C}$ is the Fredholm operator that was alluded to at the outset. There is an analogous Fredholm version of $\mathcal{D}_{C}$ in the case when $\varphi_{0}$ is not an immersion; it is described for example in Section 4 of [HT1].

The interest in these operators stems from the following fact: The set of irreducible, pseudoholomorphic subvarieties has a topology whereby a neighborhood of any given such subvariety $C$ is homeomorphic to the zero locus of a smooth map from a ball in $\operatorname{Ker}\left(\mathcal{D}_{C}\right)$ to $\operatorname{Coker}\left(\mathcal{D}_{C}\right)$. 
A by-now standard argument using the Smale-Sard theorem can be used to prove the following:

Lemma 2.3. There is a residual subset $\mathcal{J}_{0 f} \subset \mathcal{J}_{f}$ of almost complex structures with the following property: If $J \in \mathcal{J}_{0 f}$, and if $C$ is an irreducible, $J$-pseudoholomorphic subvariety, then $\mathcal{D}_{C}$ has trivial cokernel.

In terms of this, the first criterion for $J$ to be in $\mathcal{J}_{1 f}$ is:

$$
[\mathrm{J} 1]: J \in \mathcal{J}_{0 f} \text {. }
$$

Part 3. The set of almost complex structures $\mathcal{J}_{1 f}$. This part describes the second sort of criteria for membership in $\mathcal{J}_{1 f}$. Assume that $J \in \mathcal{J}_{0 f}$. The criteria here involve the ends of pseudoholomorphic subvarieties that comprise the set $\mathcal{M}_{1}\left(\Theta, \Theta^{\prime}\right)$ for pairs $\Theta, \Theta^{\prime}$ in the set $\mathcal{A}$. To say more, suppose that $\Sigma \in \mathcal{M}_{1}\left(\Theta, \Theta^{\prime}\right)$. The fact that $\Sigma$ is in a one-dimensional moduli space implies that there is a unique $(C, m) \in \Sigma$ with such that the submanifold $C$ not $\mathbb{R}$-invariant. Let $\mathcal{E} \subset C$ denote an end. Reintroduce the notation $q_{\mathcal{E}}, \lambda_{q_{\mathscr{E}}}$, and $\varsigma_{q_{\mathcal{E}}}$ from the discussion of (1.7); and define a subset $\operatorname{div}_{\mathcal{E}} \subset\left\{1, \ldots, q_{\mathcal{E}}\right\}$ as follows: An integer $q$ is in $\operatorname{div}_{\mathcal{E}}$ if either of the following is true:

(1) $q=q_{\mathcal{E}}$,

(2) $q$ is a proper divisor of $q_{\mathcal{E}}$, and there is a $(2 \pi q)$-periodic eigenfunction $\varsigma_{q}$ of the operator in (1.8) with eigenvalue $\lambda_{q}$ of the same sign as $\lambda_{q_{E}}$, such that $\left|\lambda_{q_{E}}\right|>\left|\lambda_{q}\right|>0$.

Note that the sign in item (2) above is positive or negative, respectively, when $\mathcal{E}$ is a positive or negative end. We now state the other criteria for $J$ to be in $\mathcal{J}_{1 f}$ :

For any choice of $\Theta, \Theta^{\prime}, \Sigma, C$ described above,

[J2a]: $\operatorname{div}_{\mathcal{E}}=\left\{q_{\mathcal{E}}\right\}$ for any end $\mathcal{E}$ of $C$.

[J2b]: Fix any two distinct ends of $C, \mathcal{E}$ and $\mathcal{E}^{\prime}$, which are both positive or both negative such that their respective constant $s \in \mathbb{R}$ slices converge as $|s| \rightarrow \infty$ to the same periodic orbit in $M$, and such that $q_{\mathcal{E}}=q_{\mathcal{E}^{\prime}}=: q$. Fix respective $2 \pi q$-periodic eigenfunctions $\varsigma_{q_{\mathscr{E}}}$, $\varsigma_{q_{\mathcal{E}^{\prime}}}$ for the $\mathcal{E}$ and $\mathcal{E}^{\prime}$ 's versions of the operator (1.8). Then $\varsigma_{q_{\mathcal{E}}}(t) \neq$ $\varsigma_{q_{\mathcal{E}^{\prime}}}(t+2 \pi k)$ for any integer $k$.

Definition 2.2. $\mathcal{J}_{1 f}$ is the set of almost complex structures $J$ satisfying all three conditions [J1], [J2a], [J2b] above.

The arguments in Sections 3 and 4 of [HT1] can be adapted with only notational changes to prove that the set $\mathcal{J}_{1 f}$ is a residual subset of $\mathcal{J}_{0 f}$. By way of a parenthetical remark with regards to [J2a], Section 3 in [HT1] shows that $\lambda_{q_{\mathcal{E}}}$ is the smallest positive/largest negative eigenvalue of a $2 \pi q_{\mathcal{E}^{-}}$ periodic eigenfunction. Moreover, the condition that $\Sigma$ have periodic Floer homology index 1 implies the following: Let $q$ denote a proper divisor of $q_{\mathcal{E}}$, 
and let $\lambda$ denote the eigenvalue of a $2 \pi q$-periodic eigenfunction with the sign as $\lambda_{q_{E}}$. Then $|\lambda|>\left|\lambda_{q_{E}}\right|$.

Part 4. The modified almost complex structure $J^{\prime}$ The next lemma describes a modified version of the given almost complex structure, $J$; this is to go with the modified version of $f$ provided by Lemma 2.1 and the modified version of $\partial_{t}$ provided by Lemma 2.2.

Lemma 2.4. Given an integer $N$, there exists $\kappa>1$ with the following significance: Fix $\delta<\kappa^{-1}$ and let $f^{\prime}$ denote a map given by Lemma 2.1 . Define $\partial_{t^{\prime}}$ from $f^{\prime}$ as done subsequent to Lemma 2.2. Then there is an almost complex structure, $J^{\prime}$, on $T(\mathbb{R} \times M)$ with the properties listed below:

- $J^{\prime} \partial_{s}=\partial_{t^{\prime}}$.

- $J^{\prime}$ is tamed by $d s \wedge d t+w_{f}$.

- Fix $\gamma \in \Lambda_{N}$ and extend Lemma 2.2's map $\varphi_{\gamma}$ as a map $\hat{\varphi}_{\gamma}: \mathbb{R} \times\left(S^{1} \times D\right) \rightarrow \mathbb{R} \times M, \quad(\sigma,(\tau, z)) \mapsto\left(s=q \sigma, \varphi_{\gamma}(\tau, z)\right)$.

Then $J^{\prime}\left(\hat{\varphi}_{\gamma}\right)_{*} \partial_{z}=\left(\hat{\varphi}_{\gamma}\right)_{*} \mathrm{i} \partial_{z}$ on a neighborhood of the $z=0$ locus.

- $J^{\prime} \in \mathcal{J}_{1 f^{\prime}}$.

- For any pair of periodic Floer homology generators, there is a canonical 1-1 correspondence between the J-pseudoholomorphic curves that contribute to the periodic Floer homology differential and those that are $J^{\prime}$-pseudoholomorphic. This correspondence is such that partnered curves contribute identical \pm 1 weights to the respective differentials.

This lemma is proved in Section 2.4.

With $N$ given and a choice of $\delta$ obeying the conditions of Lemmas 2.1-2.4, fix $f^{\prime}$ as in Lemma 2.1 and $J^{\prime}$ as in Lemma 2.4. The pair $\left(f^{\prime}, J^{\prime}\right)$ is said to be an $(\delta, N)$-approximation to $(f, J)$. The following is a direct corollary of Lemmas 2.1 and 2.4:

Proposition 2.1. Fix a pair $(f, J)$ with $f: F \rightarrow F$ satisfying (1.1) and $J \in \mathcal{J}_{1 f}$. Use the latter to define the chain complex and differential for periodic Floer homology. Then there exists an integer $N_{*} \geq 1$ such that $\forall N \geq N_{*}$, there exists an $\delta_{N} \in \mathbb{R}$ with the following significance:

Given any $\delta \in\left(0, \delta_{N}\right)$ and an $(\delta, N)$-approximation, $\left(f^{\prime}, J^{\prime}\right)$, to $(f, J)$, the tautological identification of the respective generators induces a canonical isomorphism from the $f$-version of the periodic Floer chain complex to the $f^{\prime}$-version, that intertwines the corresponding differentials. In particular, the two versions of periodic Floer homology are canonically isomorphic.

Remark. The definition of periodic Floer homology that was given originally in $[\mathbf{H 1}]$ restricted the pair $(f, J)$ to those where $f$ obeys the fifth item of Lemma 2.1 and where $J$ near each $\gamma \in \Lambda_{N}$ version of $\mathbb{R} \times \gamma$ is constant when written in a suitable coordinate chart. This understood, Proposition 2.1 can 
be viewed as the assertion that the periodic Floer homology for any pair $(f, J)$ can be computed using a pair of the sort described in $[\mathbf{H 1}]$.

2.3. Preliminary constructions. The proofs of Lemmas $2.1-2.4$ require some preliminary constructions which will be used in the definitions of $f^{\prime}$ and $\varphi_{\gamma}$. These are given in the subsequent two parts of this subsection.

Part 1. The tubular neighborhood maps $\varphi_{\gamma}$. Fix $\gamma \in \Lambda_{N}$. This first part describes a tubular neighborhood map for $\gamma$ that is shown in Section 2.4 to satisfy the requirements of Lemma 2.2. In what follows, $q$ denotes the period of $\gamma$.

Let $z=\left(z_{1}, z_{2}\right)$ denote the symplectic coordinates centered on the corresponding fixed point $\mathrm{p}$ of $f$ as described in Lemma 2.1. Use these coordinates to identify a neighborhood of $\mathrm{p} \in F$ with a small radius disk $D^{\prime} \subset \mathbb{R}^{2}$ about the origin. This then gives a smooth map $\varphi_{0}:[0,2 \pi] \times D^{\prime} \rightarrow M$ that embeds $[0,2 \pi) \times D^{\prime}$ and is such that

$$
\left(\varphi_{0}\right)_{*} \partial_{\tau}=\partial_{t} \quad \text { and } \quad \varphi_{0}(2 \pi, z)=\varphi_{0}\left(0, f^{q}(z)\right) .
$$

The map $z \mapsto f^{q}(z)$ maps $D^{\prime}$ to $\mathbb{R}^{2}$ and fixes the origin. It also preserves the area-form $w_{F}$. This understood, there exists a homotopy of area-preserving maps,

$$
\left\{\psi_{\tau}(z): D^{\prime \prime} \rightarrow \mathbb{R}^{2}\right\}_{\tau \in[0,2 \pi]},
$$

where $D^{\prime \prime}$ is a disk $D^{\prime \prime} \subset D^{\prime}$, and $\psi_{\tau}$ is such that:

- $\psi_{\tau}(0)=0$ for all $\tau$,

- $\psi_{\tau}(z)=z$ near $\tau=0$, and

- $\psi_{\tau}(z)=f^{-q}(z)$ near $\tau=2 \pi$.

Let $D^{*} \subset D^{\prime \prime}$ denote a small radius disk about the origin such that $D^{*}$ is in $\psi_{\tau}\left(D^{\prime \prime}\right)$ for all $\tau$. Define the map

$$
\varphi_{1}:[0,2 \pi] \times D^{*} \rightarrow M, \quad(\tau, z) \mapsto \varphi_{0}\left(\tau, \psi_{\tau}(z)\right) .
$$

By construction, $\varphi_{1}(0, z)=\varphi_{1}(2 \pi, z)$. As a consequence, $\varphi_{1}$ defines a map from $S^{1} \times D^{*}$ into $M$ such that $\varphi_{1}^{*} d t=q d \tau$.

By assumption, $J$ maps the kernel of $\pi_{*}$ to itself along $\gamma$. It is also the case that the quadratic form $w_{F}(\cdot, J(\cdot))$ is positive on the kernel of $\pi_{*}$ along $\gamma$. As a consequence, the action of $J$ along $\gamma$ on $\varphi_{1_{*}} \partial_{z}$ is such that

$$
\left(\varphi_{1}^{-1}\right)_{*} J\left(\varphi_{1}\right)_{*} \partial_{z}=\mathrm{i}\left(1+|v|^{2}\right)^{1 / 2} \partial_{z}+v \partial_{\bar{z}},
$$

where $v$ here is a smooth map from $S^{1}$ to $\mathbb{C}$. This understood, we may define:

Definition 2.3. Set $\varphi_{\gamma}: S^{1} \times D^{*} \rightarrow M$ to be the map that sends any given point $(\tau, z)$ to $\varphi_{1}\left(\tau, V_{\tau} z\right)$, where the map $\tau \mapsto V_{\tau}$, from $S^{1}$ to $\operatorname{Sl}(2 ; \mathbb{R})$ is chosen so that $\varphi_{\gamma}$ satisfies the following properties: 
- The vector field $q^{-1} \partial_{t}$ is the push-forward via $\varphi_{\gamma}$ of a vector field on $S^{1} \times D^{*}$ that can be written as

$$
\partial_{\tau}-2 \mathrm{i}(\nu z+\mu \bar{z}+\mathfrak{r}) \partial_{z}+2 \mathrm{i}(\nu \bar{z}+\bar{\mu} z+\overline{\mathfrak{r}}) \partial_{\bar{z}},
$$

where $\nu$ is an $\mathbb{R}$-valued function on $S^{1}$ and $\mu$ is a $\mathbb{C}$-valued function. Meanwhile $\mathfrak{r}$ is such that its absolute value is bounded by $\kappa|z|^{2}$, and its derivatives are bounded in absolute value by $\kappa|z|$ for a positive constant $\kappa$.

- $J\left(\varphi_{\gamma}\right)_{*} \partial_{z}=\left(\varphi_{\gamma}\right)_{*} \mathrm{i} \partial_{z}$ along $\gamma$.

In what follows, $F$ is identified with $\pi^{-1}(0) \subset M$ and $\varphi_{\gamma}$ is used to identify $\{2 \pi k / q\} \times D^{*} \subset S^{1} \times D^{*}$ with a disk in $F$, for any given $k \in\{0, \ldots, q\}$. Granted these identifications, then $\left(\varphi_{\gamma}\right)^{*} f$ on a small radius, concentric subdisk in the $\tau=2 \pi k / q$ disk inside $S^{1} \times D^{*}$ is obtained from (2.5) by following the integral curves of (2.5) from this subdisk to where they intersect the $\tau=2 \pi(k+1) / q$ disk in $S^{1} \times D^{*}$.

Part 2. The maps $u_{\tau}: D \subset F \rightarrow F$. This part constructs an $S^{1}$-parametrized family of area-preserving maps $\mathbf{u}_{\tau}$ from a certain disk in $F$ to $F$. This family is used to construct the map $f^{\prime}$.

To start, introduce $(\nu, \mu)$ to denote the pair of functions that arise when writing $q^{-1}\left(\varphi_{\gamma}^{-1}\right)_{*} \partial_{t}$ as in (2.5). There exists a one-parameter set, $\left\{\left(\nu^{\lambda}, \mu^{\lambda}\right)\right\}_{\lambda \in[0,1]}$, of respectively $\mathbb{R}$-valued and $\mathbb{C}$-valued functions on $S^{1}$ satisfying the following:

- $\left(\nu^{0}, \mu^{0}\right)=\left(\nu_{\gamma}, \mu_{\gamma}\right)$, and $\left(\nu^{1}, \mu^{1}\right)=(\nu, \mu)$.

- Let $K^{\lambda} \in \mathrm{Sl}(2 ; \mathbb{R})$ be defined so that given $z \in \mathbb{R}^{2}, K^{\lambda} \cdot z$ is the value at $\tau=2 \pi$ of the solution to the equation

$$
\frac{\mathrm{i}}{2} \frac{d}{d \tau} \eta+\nu^{\lambda} \eta+\mu^{\lambda} \bar{\eta}=0, \quad \eta(0)=z .
$$

Then each $\lambda \in[0,1]$ version of $K^{\lambda}$ is conjugate in $\mathrm{Sl}(2 ; \mathbb{R})$ to $K_{\gamma}$.

Fix such a one-parameter family.

Reintroduce the function $\chi$ from Section 1.4. Fix $\rho_{0} \in\left(0, \delta^{16}\right)$ and set $\lambda^{*}$ to be the function on $\mathbb{R}^{2}$ given by $\lambda^{*}(z)=\chi\left(\ln |z| / \ln \rho_{0}\right)$. Introduce respective $\mathbb{R}$-valued and $\mathbb{C}$-valued functions on $S^{1} \times D$ given at $(\tau, z)$ by $\left(\nu^{\lambda^{*}}, \mu^{\lambda^{*}}\right)$. With these function in hand, set

$$
\mathfrak{r}^{(1)}=\left(\nu^{\lambda^{*}}-\nu_{\gamma}\right) z+\left(\mu^{\lambda^{*}}-\mu_{\gamma}\right) \bar{z}+\lambda^{*} \mathfrak{r}+\mathfrak{e},
$$

where $\mathfrak{r}$ also comes from (2.5), and where $\mathfrak{e}$ is a smooth map from $S^{1} \times D^{*}$ to $\mathbb{C}$ with the following properties:

- $\mathfrak{e}$ is supported where $|z| \in\left(\rho_{0}^{7 / 16}, \rho_{0}^{5 / 16}\right)$.

- $\mathfrak{e}$ and its derivatives to order 100 are bounded by $\rho_{0}^{1000}$. 
The term $\mathfrak{e}$ is used subsequently as a source of "suitably generic" perturbations.

Choose $\delta$ small enough so that the disk in $\mathbb{C}$ about the origin with radius $\delta$ lies in $D^{*}$. There exists a constant $c_{0}$ so that over the disk $D \subset D^{*}$ of radius $c_{0}^{-1} \delta_{*}$, the following family of maps $\left\{\mathbf{u}_{\tau}^{(1)}: D \rightarrow D^{*}\right\}_{\tau \in[0,2 \pi]}$ is well-defined: Given $z \in \mathbb{C}$, solve the differential equation

$$
\frac{\mathrm{i}}{2} \frac{d}{d \tau} \eta+\nu_{\gamma} \eta+\mu_{\gamma} \bar{\eta}+\mathfrak{r}^{(1)}(\eta)=0, \quad \eta(0)=z,
$$

and set

$$
\mathrm{u}_{\tau}^{(1)}(z):=\eta(\tau)
$$

This map has the following three properties:

- There is a disk $D^{(1)} \subset D$ centered at the origin of radius less than $\rho_{0}^{1 / 2}$ such that $\mathbf{u}_{\tau}^{(1)}$ acts on a neighborhood of $D^{(1)}$ as multiplication by the matrix $U(\tau)$ that is constructed in Section 2.1 from the solutions to the linear equation depicted in (2.3).

- There is a disk $D^{(2)} \subset D$ of radius less than $\rho_{0}^{1 / 4}$ that contains $D^{(1)}$ and is such that $u_{\tau}^{(1)}$ acts on a neighborhood of $D-D^{(2)}$ as an areapreserving map. Furthermore, if $k \in\{0, \ldots, q-1\}$, then $\mathbf{u}_{\tau=2 \pi k / q}^{(1)}$ on $D-D^{(2)}$ is given by $\varphi_{\gamma}^{*} f$ on $\{2 \pi k / q\} \times\left(D-D^{(2)}\right)$.

- Each $\mathbf{u}_{\tau}^{(1)}$ is nearly area-preserving in the sense that its pull-back of the area form $\frac{i}{2} d z \wedge d \bar{z}$ can be written as

$$
\left(1+\mathfrak{w}_{\tau}\right) \frac{\mathrm{i}}{2} d z \wedge d \bar{z}
$$

where the error term, $\mathfrak{w}_{\tau}$, is supported in $D^{(2)}-D^{(1)}$ and is such that $\left|\mathfrak{w}_{\tau}\right| \leq c_{0}\left|\ln \rho_{0}\right|^{-1}$.

Now set

$$
\mathrm{u}_{\tau}:=\varrho_{\tau} \circ \mathrm{u}_{\tau}^{(1)},
$$

where $\varrho_{\tau}$ is a diffeomorphism of $D^{*}$, which is the identity on $D^{(1)}$ and on $D-D^{(2)}$ so that the result is area-preserving.

The map $\varrho_{\tau}$ is defined using Moser's trick. To elaborate, the map $\varrho_{\tau}$ is obtained by integrating a $\tau$-dependent vector field on $D^{(2)}-D^{(1)}$ that vanishes near the boundary. This is to say that $\frac{\partial}{\partial_{\tau}} \varrho_{\tau}=\mathrm{v}_{\tau}\left(\varrho_{\tau}\right)$, where $\tau \mapsto \mathrm{v}_{\tau}$ is a vector field with compact support on $D^{(2)}-D^{(1)}$. This vector field $\mathrm{v}_{\tau}$ is chosen so that its contraction with the form in (2.8) is an anti-derivative, $\mathfrak{b}_{\tau}$, of the two-form $\frac{i}{2} \mathfrak{w}_{\tau} d z \wedge d \bar{z}$ with compact support in $D^{(2)}-D^{(1)}$. To say more about this anti-derivative, introduce the cylindrical coordinates $(\rho, \theta)$ on $D$ by writing $z=\rho e^{\mathrm{i} \theta}$. The two-form $\frac{\mathrm{i}}{2} \mathfrak{w}_{\tau} d z \wedge d \bar{z}$ can be written as 
$d\left(h_{\tau} d \theta\right)$, where

$$
h_{\tau}(\rho, \theta)=\int_{0}^{\rho} \mathfrak{w}_{\tau}(x, \theta) x d x .
$$

The function $h_{\tau}$ is zero on $D^{(1)}$ and it depends only on the angle $\theta$ on a neighborhood of $D-D^{(2)}$. The fact that $\frac{i}{2} \mathfrak{w}_{\tau} d z \wedge d \bar{z}$ has integral zero over $D^{(2)}$ implies that $h_{\tau}=\frac{\partial}{\partial \theta} u_{\tau}$ on $D-D^{(2)}$ where $u_{\tau}$ is a function only of the angle $\theta$. This understood, let $\rho_{(2)}$ denote the radius of $D^{(2)}$, and then define

$$
\mathfrak{b}_{\tau}=h_{\tau} d \theta-d\left(\left(1-\chi\left(|\cdot| / \rho_{2}\right) u_{\tau}\right) .\right.
$$

This one-form has compact support in $D-D^{(2)}$ and is such that $d \mathfrak{b}_{\tau}=$ $\frac{i}{2} \mathfrak{w}_{\tau} d z \wedge d \bar{z}$. Note for future reference that $\left|\mathfrak{b}_{\tau}\right|$ and its $\tau$-derivative are both bounded by $c_{0}\left|\ln \rho_{0}\right|^{-1}|z|$. In addition, $\left|\nabla \mathfrak{b}_{\tau}\right|$ and its $\tau$-derivative are bounded by $c_{0}\left|\ln \rho_{0}\right|^{-1}|z|$. It follows as a consequence that $\mathrm{v}_{\tau}$ and its corresponding derivatives obey similar bounds.

2.4. Proofs of Lemmas 2.1, 2.2 and 2.4. We now have the ingredients to define the modified map:

Definition 2.4. Given a pair $(\delta, N)$ of a small positive number and an integer, let $f^{\prime}: F \rightarrow F$ be the following map:

$$
f^{\prime}=\left\{\begin{array}{cc}
\left(\varphi_{\gamma}^{-1}\right)^{*} \mathrm{u}_{\tau=2 \pi k / q} & \text { on } \varphi_{\gamma}(\{2 \pi k / q\} \times D), \\
f & \forall k \in\{0, \ldots, q-1\} \text { and } \gamma \in \Lambda_{N} \\
f & \text { on the remainder of } F .
\end{array}\right.
$$

In the above, $q$ denotes the period of $\gamma$, and the parameters $\mathfrak{e}, \rho_{0}$ that enter into the definition of $\mathbf{u}_{\tau=2 \pi k / q}$ are chosen as follows:

- the perturbation term $\mathfrak{e}$ in (2.6) is chosen in a suitably generic fashion so that all periodic points of $f^{\prime}$ are non-degenerate periodic points;

- let $\rho_{0}=\delta^{32}$.

By construction, $f^{\prime}$ obeys the first two items of Lemma 2.1, and it obeys the remaining items if all periodic orbits of $f^{\prime}$ with period $N$ or less are in $f^{\prime}$ s version of $\Lambda_{N}$. To prove this, suppose that $\gamma^{\prime} \subset F$ is a periodic orbit of $f^{\prime}$ with period $q^{\prime} \in\{1, \ldots, N\}$. Let $\mathrm{p}^{\prime} \in \gamma^{\prime}$. Given that $\left|f-f^{\prime}\right| \leq c_{0} \rho_{0}^{1 / 4}$, it follows that $\left|\operatorname{dist}\left(f^{\prime q^{\prime}}\left(\mathrm{p}^{\prime}\right), \mathrm{p}^{\prime}\right)\right| \leq c_{0} \rho_{0}^{1 / 4}$ also. Thus, if $\delta<c_{0}^{-1}$, then there is an orbit $\gamma \subset \Lambda_{N}$ of order a divisor, $q$, of $q^{\prime}$, and a point $\mathrm{p} \in \gamma$ with the following property: Each $k \in\left\{1, \ldots, q^{\prime}\right\}$ version of $f^{k}(\mathrm{p})$ has distance $c_{0} \rho_{0}^{1 / 4}$ or less from both $f^{k}\left(\mathrm{p}^{\prime}\right)$ and $\left(f^{\prime}\right)^{k}\left(\mathrm{p}^{\prime}\right)$. This understood, $\mathrm{p}^{\prime}$ and its $f^{\prime}$ iterates are in the image via the tubular neighborhood map $\varphi_{\gamma}$ of the set $\bigcup_{1 \leq k \leq q}\{2 \pi k / q\} \times D$. No generality is lost by assuming that $\mathrm{p}^{\prime}$ is in the $\varphi_{\gamma}$ image of a point $(0, z) \in S^{1} \times D$. 
Let $\eta(\tau)$ denote the solution to $(2.7)$ with $\eta(0)=z$ and set the constant

$$
\lambda=\lambda^{*}(z) .
$$

Given that $\eta$ obeys (2.7), it follows that

$$
c_{0}^{-1}|z| \leq|\eta| \leq c_{0}|z|
$$

at all $\tau \in S^{1}$. As a consequence, $\left|\lambda^{*}(\eta)-\lambda\right| \leq c_{0}|\ln \delta|^{-1}$ at each $\tau \in S^{1}$. This then implies that the equation for $\eta$ can be written as

$$
\frac{\mathrm{i}}{2} \frac{d}{d \tau} \eta+\nu^{\lambda} \eta+\mu^{\lambda} \bar{\eta}+\mathfrak{x}(\eta)=0,
$$

where $|\mathfrak{x}(\eta)| \leq c_{0}|\ln \delta|^{-1}|\eta|$. Integrating (2.12) finds that

$$
\left|\left(1-\left(K^{\lambda}\right)^{q^{\prime} / q}\right) z\right| \leq c_{0}|\ln \delta|^{-1}|z| .
$$

This last inequality requires that $z=0$ when $\delta$ is small by virtue of the fact that $K^{\lambda}$ is conjugate in $\mathrm{Sl}(2 ; \mathbb{R})$ to $K_{\gamma}$ and $\operatorname{det}\left(1-\left(K_{\gamma}\right)^{q^{\prime} / q}\right) \neq 0$ for all integer multiples $q^{\prime}$ of $q$.

To obtain Lemma 2.2 , take $\mathfrak{r}^{\prime}$ to be the version of $\mathfrak{r}^{(1)}$ given by (2.6) that is used to construct $f^{\prime}$. This done, then the lemma only summarizes definitions of $\varphi_{\gamma}$ and the map $f^{\prime}$ given in the previous section.

The proof of Lemma 2.4 differs only in notation from the proof of Proposition 2.5 from [ $\mathbf{T 1}$ ] given in the latter's Appendix. The reader is referred to this same Appendix for the details. The five remarks that follows serve only to point out certain key points that enter the proof.

Remark 2.1. It is a relatively straightforward exercise with matrices to construct an almost complex structure $J^{\prime}$ that obeys all but the fifth item of Lemma 2.4, and which has the following additional properties: First, $\left|J-J^{\prime}\right|$ is zero except in the image via the maps from $\left\{\varphi_{\gamma}\right\}_{\gamma \in \Lambda_{N}}$ of the set in $S^{1} \times D$ where $|z| \leq \rho_{0}^{1 / 4}$. Second, the difference between $J$ and $J^{\prime}$ on such an image is such that $\left|J-J^{\prime}\right| \leq c_{0}|z|$. In general $\left|\nabla\left(J-J^{\prime}\right)\right|$ will be $\mathcal{O}(1)$. This last fact complicates a direct perturbation theoretic construction of the desired pairing between $J$ and $J^{\prime}$ pseudoholomorphic curves.

Remark 2.2. To see how this problem is addressed, keep in mind that the $f$ and $f^{\prime}$ versions of the set $\Lambda_{N}$ agree; as a consequence, so do the two versions of the generating set $\mathcal{A}$ for the periodic Floer homology chain complex. This understood, fix a pair $\Theta_{-}$and $\Theta_{+}$from $\mathcal{A}$ so as to consider the $J$ and $J^{\prime}$ versions of the space $\mathcal{M}_{1}\left(\Theta_{+}, \Theta_{-}\right)$. The relatively large size of $\left|\nabla\left(J-J^{\prime}\right)\right|$ precludes a direct comparison between the $J$ and $J^{\prime}$ versions of this set. Even so, an indirect comparison can be made as follows: Fix some large integer $Q$ so as to compare successive versions of $\mathcal{M}_{1}\left(\Theta_{+}, \Theta_{-}\right)$as defined by 
an ordered set, $\left\{J_{k}\right\}_{0 \leq k \leq Q}$, of almost complex structures with the following properties:

- $J_{0}=J$, and $J_{Q}$ is the desired $J^{\prime}$.

- $J_{k}=J$ on the complement of the images via the maps $\left\{\varphi_{\gamma}\right\}_{\gamma \in \Lambda_{N}}$ of where $|z| \leq \rho_{0}^{1 / 4}$.

- $\left|J_{k}-J_{k-1}\right| \leq c_{0} Q^{-1}|z|$ on the image of $\mathbb{R} \times S^{1} \times D$ of any map from the set $\left\{\varphi_{\gamma}\right\}_{\gamma \in \Lambda_{N}}$.

- $\left|\nabla\left(J_{k}-J_{k-1}\right)\right| \leq c_{0} Q^{-1}$.

- Each $\gamma \in \Lambda_{N}$ version of the cylinder $\mathbb{R} \times \gamma$ is $J_{k}$-pseudoholomorphic.

Remarks 2.3. Perturbation-theoretic tools are used to construct a 1-1 correspondence between the pseudoholomorphic curves in the respective versions of $\mathcal{M}_{1}\left(\Theta_{+}, \Theta_{-}\right)$as defined by the almost complex structures at successive steps. Likewise, perturbation theory is used to prove that paired almost pseudoholomorphic curves have equal \pm 1 weights of the sort used to compute the embedded contact homology differential.

The use of perturbative tools exploits four key facts. Here are the first three:

Fact 1. The factor $Q^{-1}$ can be very small.

Fact 2. Each $\gamma \in \Lambda_{N}$ version of the cylinder $\mathbb{R} \times \gamma$ is $J_{k}$-pseudoholomorphic for each $k$.

Fact 3. Successive versions of $\left|J_{k+1}-J_{k}\right|$ can have support in successively smaller neighborhoods of $\bigcup_{\gamma \in \Lambda_{N}} \gamma$; and these neighborhoods can be made as small as desired, independent of $Q$ and $\delta$.

To say something about the fourth key fact, suppose that $C$ is a non- $\mathbb{R}$ invariant subvariety that appears in some element from the $J_{k}$ version of $\mathcal{M}_{1}\left(\Theta_{+}, \Theta_{-}\right)$. Any given end $\mathcal{E} \subset C$ at large $|s|$ is described by (1.7) but with the functions $(\nu, \mu)$ dependent on the index $k$. This understood, here is the fourth fact:

Fact 4. There is a lower bound, independent of $k, Q$ and $\delta$ to the absolute value of the eigenvalue $\lambda_{q_{E}}$ that can appear in (1.7).

Remark 2.4. These four facts are relevant because any given non- $\mathbb{R}$ invariant, $J_{k}$-pseudoholomorphic subvariety will intersect the small neighborhoods in Fact 3 above in only two ways. First, such a subvariety, $C$, can intersect this neighborhood near the points where it intersects a cylinder of the form $\mathbb{R} \times \gamma$ with $\gamma \in \Lambda_{N}$. There are but a finite set of points where $C$ intersects any given $\mathbb{R}$-invariant cylinder. In fact, there is a bound on the number of such points that is essentially topological and so independent of $C$ and of the index $k$. In particular, if the modification from one step to the next is done in a very small neighborhood of the cylinders $\{\mathbb{R} \times \gamma\}_{\gamma \in \Lambda_{N}}$, then the region where $J_{k+1} \neq J_{k}$ will intersect all but the very large $|s|$ part of $C$ in a set of at most $c_{0}^{-1}$ disks, each with very small area. This has the following 
consequences: The subvariety $C$ is $J_{k+1}$-pseudoholomorphic except at very large $|s|$, and except in a union of at most $c_{0}^{-1}$ disks, each very small area. Moreover, $C$ is nearly $J_{k+1}$-pseudoholomorphic in each such disk if $Q$ is large and $\delta$ is small, both in a pointwise sense and in an $L^{2}$ sense.

The subvariety $C$ also intersects the region where $J_{k+1} \neq J_{k}$ at points far out on its ends, thus at very large values of $|s|$. The area of intersection here is, of course, infinite. However, the relevant versions of (1.7) are used to prove that this is of no ultimate consequence. In particular, $C$ is nearly $J_{k+1}$-pseudoholomorphic in a pointwise sense and also in an $L^{2}$ sense if $Q$ is large and $\delta$ is small.

Remark 2.5. This final remark concerns a most crucial point in the constructions from the Appendix of [T1]: The perturbative approach from this appendix will construct a partner to $C$ from the $J_{k+1}$ version of $\mathcal{M}_{1}\left(\Theta_{+}, \Theta_{-}\right)$ provided that the operator $\mathcal{D}_{C}$ from (2.4) is invertible. The four facts stated above, and Fact 4 in particular, guarantee that such is the case when $Q$ is large and $\delta$ is very small.

\section{Constructing Seiberg-Witten solutions on $M$}

Define $M$ from a given area-preserving map $f: F \rightarrow F$. The purpose of this section is to explain how the constructions in Section 3 of [T2] are used to associate solutions to certain versions of the Seiberg-Witten equations to finite sets with any given element a pair consisting of a closed integral curve of $\partial_{t}$ and a positive integer. Such an association can also be obtained from the constructions in the article $\mathrm{Gr} \Rightarrow \mathrm{SW}$ from [T6]. The constructions from Section 3 of [T2] differ with regard to certain details from those in the $\mathrm{Gr} \Rightarrow \mathrm{SW}$ article, but they are identical in spirit.

The relation between the solutions to the Seiberg-Witten equations and sets of closed integral curves is described in Theorem 3.1 of the upcoming Section 3.4. The intervening subsections set the stage.

3.1. Vortices. This subsection summarizes some of what is said in Section 2 of [T2] about the vortex equations on $\mathbb{C}$. These are equations for a pair $(A, \alpha)$ where $A$ is a connection on the product $\mathbb{C}$-bundle over $\mathbb{C}$ and $\alpha$ is a section of this bundle. These equations read

$$
* F_{A}=-\mathrm{i}\left(1-|\alpha|^{2}\right) \text { and } \bar{\partial}_{A} \alpha=0 .
$$

Here, $F_{A}$ denotes the curvature two-form of $A$ and $\bar{\partial}_{A}$ is the d-bar operator defined by $A$. These equations are augmented with the constraint that (1$\left.|\alpha|^{2}\right)$ be integrable. Solutions $(A, \alpha)$ and $\left(A^{\prime}, \alpha^{\prime}\right)$ are deemed gauge equivalent when there exists a map $u: \mathbb{C} \rightarrow S^{1}$ such that $A^{\prime}=A-u^{-1} d u$ and $\alpha^{\prime}=u \alpha$.

The space of equivalence classes of solutions has path components $\left\{\mathfrak{C}_{m}\right\}_{m=0,1, \ldots}$. The space $\mathfrak{C}_{0}$ consists of the gauge equivalence classes of the 
pair $\left(A_{I}, \alpha=1\right)$ where $A_{I}$ is the product connection on $\mathbb{C} \times \mathbb{C}$. Meanwhile, any given $m \geq 1$ version of $\mathfrak{C}_{m}$ consists of the gauge equivalence classes of solutions to (3.1) with $\alpha$ having $m$ zeros counting multiplicity. Each such $\mathfrak{C}_{m}$ has the structure of a complex manifold. The complex structure is such that the holomorphic tangent space to $\mathfrak{C}_{m}$ at the equivalence class of some given solution $\mathfrak{c}=(A, \alpha)$ has a canonical identification with the $L^{2}$ kernel of the operator:

$$
\begin{aligned}
\vartheta_{\mathfrak{c}}: C^{\infty}(\mathbb{C} ; \mathbb{C} \oplus \mathbb{C}) & \rightarrow C^{\infty}(\mathbb{C} ; \mathbb{C} \oplus \mathbb{C}), \\
(x, \iota) & \mapsto\left(\partial x+2^{-1 / 2} \bar{\alpha} \iota, \bar{\partial}_{A} \iota+2^{-1 / 2} \alpha x\right) .
\end{aligned}
$$

Here, $\partial$ denotes $\frac{\partial}{\partial z}$. The $L^{2}$ kernel of $\vartheta_{\mathfrak{c}}$ is denoted by $\operatorname{Ker}\left(\vartheta_{\mathfrak{c}}\right)$ in what follows. The space $\mathfrak{C}_{m}$ is biholomorphic to $\mathbb{C}^{m}$; and the collection of functions $\left\{\sigma_{q}\right\}_{1 \leq q \leq m}$ on $\mathfrak{C}_{m}$ given by

$$
\sigma_{q}=\frac{1}{2 \pi} \int_{\mathbb{C}} z^{q}\left(1-|\alpha|^{2}\right)
$$

give a set of holomorphic coordinates on $\mathfrak{C}_{m}$.

The inner product on $L^{2}(\mathbb{C} ; \mathbb{C} \oplus \mathbb{C})$ gives $\mathfrak{C}_{m}$ a Kähler metric. To be explicit, the induced Hermitian inner product on $T_{1,0} \mathfrak{C}_{m}$ is such that the square of the norm of any given element $(x, \iota)$ in $\operatorname{Ker}\left(\vartheta_{\mathfrak{c}}\right)$ is

$$
\frac{1}{\pi} \int_{\mathbb{C}}\left(|x|^{2}+|\iota|^{2}\right)
$$

Except for the $n=1$ case, this Kähler metric is not the pull-back of the standard metric on $\mathbb{C}^{m}$ via the holomorphic identification that is defined by the functions $\left(\sigma_{1}, \ldots, \sigma_{m}\right)$.

The action of $S^{1}$ on $\mathbb{C}$ as multiplication by the unit complex numbers induces an isometric action of $S^{1}$ on $\mathfrak{C}_{m}$. This action has a unique fixed point, this being the unique solution to (3.1) whose version of $\alpha$ vanishes at the origin with degree $m$. This point in $\mathfrak{C}_{m}$ is called the symmetric vortex.

Of interest in what follows are certain sorts of dynamical flows on $\mathfrak{C}_{m}$. To say more, fix a pair $(\nu, \mu)$ of maps from $S^{1}$ to $\mathbb{R}$ and $\mathbb{C}$. Use the latter to define the real valued function

$$
\hat{\hbar}=\hbar_{(\nu, \mu)}:=\frac{1}{4 \pi} \int_{\mathbb{C}}\left(2 \nu|z|^{2}+\left(\mu \bar{z}^{2}+\bar{\mu} z^{2}\right)\right)\left(1-|\alpha|^{2}\right) .
$$

on $S^{1} \times \mathfrak{C}_{m}$. View this as a one-parameter family of functions on $\mathfrak{C}_{m}$. Use the Kähler two-form to define the corresponding one-parameter family of Hamiltonian vector fields. Of interest are the closed, integral curves of this family. These are maps $\mathfrak{c}: S^{1} \rightarrow \mathfrak{C}_{m}$ that obey at each $\tau \in S^{1}$ the equation

$$
\frac{\mathrm{i}}{2} \mathfrak{c}^{\prime}+\left.\nabla^{(1,0)} \mathfrak{h}\right|_{\mathfrak{c}}=0
$$


where $\mathfrak{c}^{\prime}$ is shorthand for the $(1,0)$ part of $\mathfrak{c}_{*} \frac{d}{d \tau}$, and where $\nabla^{(1,0)} \mathfrak{f}$ denotes the $(1,0)$ part of the gradient of $f$.

A solution to (3.6) is said to be non-degenerate when a certain auxiliary, self-adjoint operator has trivial kernel. To elaborate, suppose for the moment that $\mathfrak{c}: S^{1} \rightarrow \mathfrak{C}_{m}$ is any given map. Associate to the latter the symmetric operator

$$
\zeta \mapsto \frac{\mathrm{i}}{2} \nabla_{\tau} \zeta+\left.\left(\nabla_{\zeta_{\mathbb{R}}} \nabla^{(1,0)} \hbar\right)\right|_{\mathfrak{c}}
$$

on $C^{\infty}\left(S^{1} ; \mathfrak{c}^{*} T_{1,0} \mathfrak{C}_{n}\right)$. Here, $\nabla_{\tau}$ denotes the covariant derivative on $C^{\infty}\left(S^{1}\right.$; $\left.\mathfrak{c}^{*} T_{1,0} \mathfrak{C}_{n}\right)$ as defined by the pull back of the Levi-Civita connection on $T_{1,0} \mathfrak{C}_{n}$. Meanwhile, $\left.\left(\nabla_{\zeta_{\mathbb{R}}} \nabla^{(1,0)} h_{)}\right)\right|_{\mathfrak{c}}$ denotes the covariant derivative at $\mathfrak{c}$ along the vector defined by $\zeta$ in $\left.T \mathfrak{C}_{n}\right|_{\mathfrak{c}}$ of the vector field $\nabla^{(1,0)} \hat{h} \in C^{\infty}\left(\mathfrak{C}_{n} ; T_{1,0} \mathfrak{C}_{n}\right)$. A solution to (3.6) is non-degenerate when the operator depicted in (3.7) has trivial kernel.

The subsequent lemma plays a key role in what follows.

Lemma 3.1. Fix a positive integer $m$ and a pair $(\nu, \mu): S^{1} \rightarrow \mathbb{R} \times \mathbb{C}$ whose version of (1.8) has trivial $2 \pi q$ periodic kernel for each $q \in\{1, \ldots, m\}$. Then the space of solutions to the corresponding version of (3.6) is compact; and it is finite if all solutions are non-degenerate.

The proof of this lemma is identical to the proof given in Section $2 \mathrm{~b}$ of [T4] of the latter's Proposition 2.1.

All solutions to (3.6) are known in special cases. What follows summarizes what is said in Lemmas 2.1-2.3 of [T2].

Lemma 3.2. (a) Fix a pair $(\nu, \mu)$ with $\nu$ a real valued function on $S^{1}$ and $\mu$ a $\mathbb{C}$-valued function. Use this pair to define the operator, $\mathcal{P}: C^{\infty}(\mathbb{R}, \mathbb{C}) \rightarrow C^{\infty}(\mathbb{R}, \mathbb{C})$ by the rule $\eta \mapsto \frac{\mathrm{i}}{2} \frac{d}{d \tau} \eta+\nu \eta+\mu \bar{\eta}$. Assume that 0 is the only $2 \pi$-periodic element in the kernel of $\mathcal{P}$. Then there is a unique solution to the corresponding $m=1$ version of (3.6), which is the constant map to the symmetric vortex. This solution is non-degenerate.

(b) Fix an irrational number $\mathrm{R}$ and set $(\nu=\mathrm{R}, \mu=0)$. Then there is a unique solution in $m \geq 1$ version of $\mathfrak{C}_{m}$ to the corresponding version of (3.6). This is the constant map from $S^{1}$ to the symmetric vortex $\mathfrak{c}_{0} \in \mathfrak{C}_{m}$. This solution is non-degenerate.

(c) Fix $\mathrm{R} \in \frac{1}{2} \mathbb{Z}$ and a positive number $z$. Set $\left(\nu=\frac{1}{2} \mathrm{R}, \mu=-\frac{\mathrm{i}}{4 \pi} z \mathrm{e}^{2 \mathrm{iR} \tau}\right)$. Then there is a unique solution to the corresponding $m=1$ version of (3.6). This is the map to the symmetric vortex. This solution is nondegenerate. On the other hand, there are no solutions to the $m>1$ versions.

Fix $m \geq 1$ and suppose that $(\nu, \mu)$ are as described in Lemma 3.1. In such circumstance, any given non-degenerate solution to the corresponding 
version of (3.6) can be assigned an integer degree. To do this, use the given pair $(\nu, \mu)$ to define the operator $\mathcal{P}$ in Lemma 3.2. Let $\eta(\tau)$ denote a nontrivial element in the kernel of $\mathcal{P}$. Then $\eta(\tau)$ can be written as

$$
\eta(\tau)=U(\tau)\left(\eta_{0}\right)
$$

where $U(\tau)$ is an $\mathbb{R}$-linear map from $\mathbb{C}$ to $\mathbb{C}$ that defines a matrix in $\mathrm{Sl}(2 ; \mathbb{R}$ when $\mathbb{C}$ is written as $\mathbb{R}^{2}$. The pair $(\nu, \mu)$ is said to be elliptic with rotation number a given real number $\mathrm{R} \notin \mathbb{Z}$ when $U$ on $[0,2 \pi]$ defines a path in $\mathrm{Sl}(2 ; \mathbb{R})$ that is homotopic in $\mathrm{Sl}(2 ; \mathbb{R})$ via a homotopy $\left\{U_{x}(\cdot)\right\}_{x \in[0,1]}$ with the following three properties: The matrix $U_{x}(0)$ is the identity, the matrix $U_{x}(2 \pi)$ is conjugate in $\mathrm{Sl}(2 ; \mathbb{R})$ to $U(2 \pi)$, and the path $U_{x=1}(\cdot)$ is the rotation with smooth angle parametrization $\tau \mapsto 2 \pi \mathrm{R} \tau$. It is assumed in what follows that $\mathrm{R}$ is irrational when $(\nu, \mu)$ are elliptic. The pair $(\nu, \mu)$ is said to be hyperbolic when the absolute value of the trace of $U(2 \pi)$ is greater than 2 . In this case, there is a one-parameter family of pairs of function

$$
\left\{\left(\nu_{x}, \mu_{x}\right): S^{1} \rightarrow \mathbb{R} \times \mathbb{C}\right\}_{x \in[0,1]}
$$

with the following three properties:

- $\left(\nu_{0}, \mu_{0}\right)=(\nu, \mu)$.

- Each pair $\left(\nu_{x}, \mu_{x}\right)$ has a corresponding $U_{x}(2 \pi)$ whose trace has absolute value at least 2 .

- The path $\tau \mapsto U_{x=1}(\tau)$ as defined by the pair $\left(\nu_{1}, \mu_{1}\right)$ is the rotation with smooth angle parametrization $\tau \mapsto 2 \pi \mathrm{R} \tau$ with rotation number $\mathrm{R} \in \frac{1}{2} \mathbb{Z}$.

Granted the preceding, let

$$
\mathfrak{c}_{0}: S^{1} \rightarrow \mathfrak{C}_{m}
$$

denote the constant map to the symmetric vortex in $\mathfrak{C}_{m}$. Associate to $\mathfrak{c}_{0}$ the operator

$$
\mathcal{R}: C^{\infty}\left(S^{1} ;\left.T \mathfrak{C}_{m}\right|_{\mathfrak{c}_{0}}\right) \rightarrow C^{\infty}\left(S^{1} ;\left.T \mathfrak{C}_{m}\right|_{\mathfrak{c}_{0}}\right),
$$

that is defined as follows:

- If $(\nu, \mu)$ is elliptic with rotation number $\mathrm{R}$, this operator is the version of (3.7) with $\hbar=\hbar_{\left(\frac{1}{2} \mathrm{R}, 0\right)}$.

- If $(\nu, \mu)$ is hyperbolic with rotation number $\mathrm{R}$, the operator $\mathcal{R}$ is the version of (3.7) with $\hbar=h_{\left(\frac{1}{2}\left(\mathrm{R}-\mathrm{R}^{\prime}\right), 0\right)}$, where $\mathrm{R}^{\prime}>0$ is an irrational number such that $m \mathrm{R}^{\prime} \ll 1$.

Lemma 2.4 in [ $\mathbf{T 2}$ ] asserts that the operator $\mathcal{R}$ has trivial kernel in these cases.

Definition 3.1. Let $(\nu, \mu)$ be as in Lemma 3.1, and suppose that $\mathfrak{c}: S^{1} \rightarrow$ $\mathfrak{C}_{m}$ is non-degenerate in the sense that (3.7) has trivial kernel. Then the degree of $\mathfrak{c}$, denoted

$$
\operatorname{deg}_{\mathfrak{C}}(\mathfrak{c})=\operatorname{deg}_{\mathfrak{C}}(\mathfrak{c},(\nu, \mu))
$$


in the following, is the spectral flow from the operator $\mathcal{R}$ to $\mathfrak{c}$ 's version of (3.7).

For the definition of this spectral flow, see, e.g., Section 2 of [T8]).

Lemma 3.3. Let $(\nu, \mu)$ be the pair $\left(\nu_{\gamma}, \mu_{\gamma}\right)$ that appears in Definition 2.1. Fix $m \in\{1,2, \ldots\}$ when $(\nu, \mu)$ are elliptic and set $m=1$ when $\gamma$ is hyperbolic. Then the map to the symmetric vortex $\mathfrak{c}_{0}: S^{1} \rightarrow \mathfrak{C}_{m}$ has degree

$$
\operatorname{deg}_{\mathfrak{C}}\left(\mathfrak{c}_{0},\left(\nu_{\gamma}, \mu_{\gamma}\right)\right)= \begin{cases}0 & \text { when } \gamma \text { is elliptic or negative hyperbolic, } \\ 1 & \text { when } \gamma \text { is positive hyperbolic. }\end{cases}
$$

Proof. When $\gamma$ is elliptic, this is true by definition. When $\gamma$ is hyperbolic and $m=1$, the operator in (3.7) when written using the (3.3)'s coordinate $\sigma_{1}$ to identify $\mathfrak{C}_{1}$ with $\mathbb{C}$ is the operator $\mathcal{P}$ that appears in Lemma 3.2. This understood, the observation about the spectral flow in the negative hyperbolic case follows from the fact that the version of $\mathcal{P}$ with $(\nu, \mu)=(\pi, 0)$ has trivial $2 \pi$-periodic kernel. Meanwhile, the calculation of the assertion about the spectral flow in the positive hyperbolic case follows by direct computation using the $[0,1]$-parametrized path $x \mapsto \mathcal{P}_{x}$ with $\mathcal{P}_{x}$ defined by $\left(\nu_{x}, \mu_{x}\right)=(-(1-x) \delta,-i \delta)$ with $\delta \in(0,1)$.

3.2. Periodic orbits and vortices. Return now to the context where $F$ is a surface with area form $w_{F}$ and $f: F \rightarrow F$ is an area-preserving map. Let $M$ denote the resulting three-manifold. Suppose that $\Theta$ is a finite set whose elements are described next. A given element is a pair $(\gamma, m)$ with $\gamma$ a closed integral curve of $\partial_{t}$ and $m$ a positive integer. Assume that distinct pairs from $\Theta$ have distinct integral curves. Note that $\Theta$ can be taken to be the empty set.

Let $(\gamma, m) \in \Theta$. Fix a tubular neighborhood map $\varphi_{\gamma}: S^{1} \times D_{\gamma} \rightarrow M$ with $D_{\gamma} \subset \mathbb{C}$ a disk about the origin. The map $\varphi_{\gamma}$ should be chosen to obey the conditions set forth by the first two items and the fourth item of Lemma 2.2. Use this map $\varphi_{\gamma}$ to define corresponding functions $(\nu, \mu)$; and let $\mathfrak{C}_{(\gamma, m)}$ denote the set of solutions to the corresponding version (3.6) with $h_{i}=h_{(\nu, \mu)}$. Let

$$
\mathfrak{C} \Theta:=\times_{(\gamma, m) \in \Theta} \mathfrak{C}_{(\gamma, m)} .
$$

Use $\mathfrak{C} \Theta^{*} \subset \mathfrak{C} \Theta$ to denote the subset of elements with the property that constituent maps $\left\{\mathfrak{c}_{\gamma}: S^{1} \rightarrow \mathfrak{C}_{m}\right\}_{(\gamma, m) \in \Theta}$ are non-degenerate.

Now suppose that a class $\Gamma \in H_{1}(M ; \mathbb{Z})$ has been specified. As in the introduction, use $d_{\Gamma}$ to denote the intersection number between $\Gamma$ and a typical fiber of the projection map $\pi$. Let $p$ denote the greatest integer divisor of the class $c_{\Gamma} \in H^{2}(M ; \mathbb{Z})$.

Let $\mathcal{Z}$ denote the set whose elements are described next. A given element is a set $\Theta$ as just described, but constrained so that the integral chain 
$\sum_{(\gamma, m) \in \Theta} m \gamma$ represents the class $\Gamma$. Note that $\mathcal{Z}=\emptyset$ if $d_{\Gamma} \leq 0$ and if $\Gamma=0$, then $\mathcal{Z}$ has only the element $\Theta=\emptyset$. Hutchings specifies in Definition 1.5 of $[\mathbf{H 1}]$ a relative $\mathbb{Z} / p \mathbb{Z}$-degree for each element $\Theta \in \mathcal{Z}$. This degree is relative in the following sense: A reference element $\Theta^{*} \in \mathcal{Z}$ is assigned degree 0 when $\mathcal{Z} \neq \emptyset$, then each $\Theta \in \mathcal{Z}$ has a well defined degree assignment in $\mathbb{Z} / p \mathbb{Z}$. This degree is denoted by $\operatorname{deg}_{p F h}(\Theta)$. If $\Theta^{\prime}$ is a second element in $\mathcal{Z}$, then the difference $\operatorname{deg}_{p F h}(\Theta)-\operatorname{deg}_{p F h}\left(\Theta^{\prime}\right)$ is the $\mathbb{Z} / p \mathbb{Z}$ reduction of what was denoted in Section 1.1 by $I\left(\Theta, \Theta^{\prime} ; Z\right)$, where $Z$ is a two-chain in $M$ with $\partial Z=\sum_{(\gamma, m) \in \Theta} m \gamma-\sum_{(\gamma, m) \in \Theta^{\prime}} m \gamma$.

The relative $\mathbb{Z} / p \mathbb{Z}$-degree for a given $\Theta \in \mathcal{Z}$ can be used to assign a relative $\mathbb{Z} / p \mathbb{Z}$-degree to each $x=\left\{\mathfrak{c}_{\gamma}\right\}_{(\gamma, m) \in \Theta} \in \mathfrak{C} \Theta^{*}$, this being

$$
\operatorname{deg}_{\mathfrak{C Z}}(x):=\operatorname{deg}_{p F h}(\Theta)+\sum_{(\gamma, m) \in \Theta} \operatorname{deg}_{\mathfrak{C}}\left(\mathfrak{c}_{\gamma}\right)
$$

3.3. The Seiberg-Witten equations. This subsection sets the stage for the definition of the relevant versions of the Seiberg-Witten equations.

3.3.1. The almost complex structure $\boldsymbol{J}$. Suppose that a non-degenerate $f$ is given to define $M$, and that $J \in \mathcal{J}_{f}$ is an $\mathbb{R}$-invariant almost complex structure on $\mathbb{R} \times M$. Recall that by definition, such a $J$ satisfies:

(1) $J \partial_{s}=\partial_{t}$ where $\partial_{t}$ is a vector field on $M$ with $\left\langle d t, \partial_{t}\right\rangle=1$ whose first return is $f$.

(2) $J$ is tamed by the form $\Omega_{F}=d s \wedge d t+w_{F}$.

Because of item (1) above, the almost complex structure $J$ is determined by its action on $\operatorname{Ker}\left(\pi_{*}\right)$. Its action here determines a pair $(\mathfrak{j}, a)$ where $\mathfrak{j}$ is an endomorphism of $\operatorname{Ker}\left(\pi_{*}\right)$ with square -1 and $a$ is a section of $\operatorname{Hom}\left(\operatorname{Ker}\left(\pi_{*}\right) ; \mathbb{R}\right)$. These are defined so that

$$
J v=\mathfrak{j} v+a(v) \partial_{s}+a(\mathfrak{j} v) \partial_{t} \quad \text { for } v \in \operatorname{Ker}\left(\pi_{*}\right) .
$$

The assertion that $J$ is tamed by $w_{0}$ is equivalent to the assertion that

$$
|a(v)|^{2}<4 w_{F}(v, \mathfrak{j} v) .
$$

It is important for what follows to note that

$$
J\left(v+a(v) \partial_{t}\right)=\mathfrak{j} v+a(\mathfrak{j} v) \partial_{t} .
$$

As a consequence, $J$ preserves the subbundle

$$
K^{-1}=\left\{v+a(v) \partial_{t}: v \in \operatorname{Ker}\left(\pi_{*}\right)\right\} .
$$

By design, this subbundle has the structure of a complex line bundle over $M$. The bundle is of course isomorphic to the kernel of $\pi_{*}$ with its complex 
structure defined by $\mathfrak{j}$. The bundle $K^{-1}$ is the kernel of the one-form

$$
\mathrm{a}=d t-a,
$$

where $a$ is viewed here and subsequently as a one-form on $M$ that annihilates $\partial_{t}$.

3.3.2. The metric $g$. The almost complex structure $J$ and the area form $w_{F}$ together define a metric $g$ on $M$ as follows:

- The vector field $\partial_{t}$ has norm 1 and is orthogonal to $K^{-1}$.

- The inner product on $K^{-1}$ is given by $w_{F}(\cdot, \mathfrak{j}(\cdot))$.

With this definition, $g$ is such that

$$
|\mathrm{a}|=1 \quad \text { and } \quad * \mathrm{a}=w_{F} .
$$

Use this metric to define the oriented orthonormal frame bundle of $M$ and to define the corresponding $\operatorname{Spin}^{\mathbb{C}}$ lifts of this frame bundle.

3.3.3. The $\operatorname{Spin}^{\mathbb{C}}$ structure. Fix a $\operatorname{Spin}^{\mathbb{C}}$-bundle $\pi: \mathcal{F} \rightarrow M$, and let

$$
\mathbb{S}=\mathcal{F} \times_{U(2)} \mathbb{C} \rightarrow M
$$

denote the corresponding $\mathbb{C}^{2}$-bundle. Clifford multiplication on $\mathbb{S}$ by the oneform a from (3.11) has eigenvalues $\pm \mathrm{i}$, and so splits $\mathbb{S}$ as a direct sum of the corresponding two eigenbundles. This splitting is written as

$$
\mathbb{S}=E \oplus E \otimes K^{-1}
$$

where the convention has $\operatorname{cl}(\mathrm{a})$ acting as i on the left most summand. The assignment of $c_{1}(E)$ to the Spin ${ }^{\mathbb{C}}$-bundle defines a $1-1$ correspondence between the set of equivalence classes of $\operatorname{Spin}^{\mathbb{C}}$-bundles over $M$ and the elements in $H^{2}(M ; \mathbb{Z})$. The canonical $\operatorname{Spin}^{\mathbb{C}}$-structure is one whose spinor bundle is splits as $\mathbb{S}_{I}=I_{\mathbb{C}} \oplus K^{-1}$, where $I_{\mathbb{C}}$ here denotes a complex line bundle that is isomorphic to the product bundle $M \times \mathbb{C}$.

3.3.4. Spin ${ }^{\mathbb{C}}$ connections and Dirac operators. As explained in Section $1 \mathrm{c}$ of the article $\mathrm{SW} \Rightarrow \mathrm{Gr}$ from [T6], there is a unique connection, $A_{K}$, on $K^{-1}$ which is characterized as follows: Fix a unit norm section, $1_{\mathbb{C}}$, of the bundle $I_{\mathbb{C}}$, and let $A_{I}$ denote the unique Hermitian connection on $I_{\mathbb{C}}$ that makes $1_{\mathbb{C}}$ covariantly constant. Use the pair $A_{K}$ and $A_{I}$ to define a connection on $\operatorname{det}(\mathbb{S})$. Then the section $\psi_{I}=\left(1_{\mathbb{C}}, 0\right)$ obeys the Dirac equation.

Because of (3.12), the connection $A_{K}$ and a Hermitian connection on $E$ together define a connection $\mathbb{A}$ on $\operatorname{det}(\mathbb{S})=E^{\otimes 2} \otimes K^{-1}$. Conversely, a connection on the latter bundle defines with $A_{K}$ a Hermitian connection on $E$. This understood, the Seiberg-Witten equations are viewed in what follows as equations for a pair $(A, \psi)$ of connection on $E$ and section of $\mathbb{S}$. 
The corresponding Dirac operator is denoted now as $D_{A}$. The space of smooth, Hermitian connections on $E$ is denoted in what follows by $\operatorname{Conn}(E)$.

3.3.5. The 3-dimensional Seiberg-Witten equations. The versions of the Seiberg-Witten equations of interest are parametrized by a number $r>$ 1. Fix such an $r$. With the metric and Spin ${ }^{\mathbb{C}}$-structure chosen previously, the corresponding equations for a pair $(A, \psi)$ from the space $\operatorname{Conn}(E) \times$ $C^{\infty}(M ; \mathbb{S}) \mathrm{read}$

$$
B_{A}=r\left(\psi^{\dagger} \tau \psi-\mathrm{ia}\right)-\frac{1}{2} B_{A_{K}}-\frac{\mathrm{i}}{2} * \wp, \quad \text { and } \quad D_{A} \psi=0 .
$$

Here, $B_{A}$ denotes the Hodge star of $A$ 's curvature two-form, and $\wp$ is a closed two-form in the cohomology class $2 \pi c_{1}(\mathfrak{s})$. Solutions $(A, \psi)$ and $\left(A^{\prime}, \psi^{\prime}\right)$ are gauge equivalent if there is a smooth map, $u$, from $M$ to $S^{1}$ such that

$$
A^{\prime}=A-u^{-1} d u \quad \text { and } \quad \psi^{\prime}=u \psi .
$$

Because $* a=w_{f}$, the equations in (3.13) correspond to a version of (1.11) with

$$
\varpi=2 r w_{f}+\wp, \quad \Psi=(2 r)^{1 / 2} \psi,
$$

and $\mathbb{A}$ defined from $A$ and $K$ as above. When $r$ is fixed, we shall use $(\mathbb{A}, \Psi)$ and $(A, \psi)$ interchangably to denote a configuration, and write the configuration space as $\operatorname{Conn}(E) \times C^{\infty}(M, \mathbb{S})$. Use $\mathrm{C}^{r}$ in what follows to denote the space of gauge equivalence classes of solutions to (3.13).

3.3.6. The relative grading. Each configuration $\mathfrak{c}=(A, \psi)$ has an associated elliptic, symmetric operator

$$
\mathfrak{L}_{\mathfrak{c}}: C^{\infty}\left(M ; \mathrm{i} T^{*} M \oplus \mathbb{S} \oplus \mathrm{i} \mathbb{R}\right) \rightarrow C^{\infty}\left(M ; \mathrm{i} T^{*} M \oplus \mathbb{S} \oplus \mathrm{i} \mathbb{R}\right),
$$

which sends any given section $(b, \eta, \phi)$ of $\mathrm{i} T^{*} M \oplus \mathbb{S} \oplus \mathrm{i} \mathbb{R}$ to the section whose respective $\mathrm{i} T^{*} M, \mathbb{S}$ and $\mathrm{i} \mathbb{R}$ components are

$$
\left\{\begin{array}{l}
* d b-d \phi-2^{-1 / 2} r^{1 / 2}\left(\psi^{\dagger} \tau \eta+\eta^{\dagger} \tau \psi\right), \\
D_{A} \eta+2^{1 / 2} r^{1 / 2}(\operatorname{cl}(b) \psi+\phi \psi), \\
* d * b-2^{-1 / 2} r^{1 / 2}\left(\eta^{\dagger} \psi-\psi^{\dagger} \eta\right) .
\end{array}\right.
$$

(Note that despite its appearance, $\mathfrak{L}_{\mathfrak{c}}$ depends only on the configuration $\mathfrak{c}=(\mathbb{A}, \Psi)$, not on $r$.) A configurationc is said to be non-degenerate when $\mathfrak{L}_{\mathfrak{c}}$ has trivial cokernel. Let $\mathfrak{c}_{-}, \mathfrak{c}_{+}$be non-degenerate configurations. Given a path $\mathfrak{d}(s)$ of configurations from $\mathfrak{c}_{-}$to $\mathfrak{c}_{+}$, let $\mathcal{J}(\mathfrak{d})$ denote the spectral flow of the associated family of operators from $\mathfrak{L}_{\mathfrak{c}_{-}}$to $\mathfrak{L}_{\mathfrak{c}_{+}}$, defined as in [T8]. For $\mathfrak{c}_{+}=u \cdot \mathfrak{c}_{-}$, one has

$$
\mathcal{J}(\mathfrak{d})=\left\langle c_{1}(\mathfrak{s}),[u]\right\rangle,
$$

where $u \in C^{\infty}\left(M ; S^{1}\right)$ is a gauge transformation and $[u]$ denotes its cohomology class:

$$
[u]:=u^{-1} d u /(2 \pi \mathrm{i}) .
$$


Thus, as promised in Section 1.2 , we may write $\mathcal{J}\left(\mathfrak{c}_{-}, \mathfrak{c}_{+} ; \mathfrak{h}\right)=\mathcal{J}(\mathfrak{d})$, which depends only on the gauge equivalence classes of $\mathfrak{c}_{-}, \mathfrak{c}_{+}$, and the relative homotopy class $\mathfrak{h}$ of $\mathfrak{d}$. Fix a non-degenerate reference configuration $\mathfrak{c}_{E}:=$ $\left(A_{E}, \psi_{E}\right)$. Given a non-degenerate configuration $\mathfrak{c}$, let

$$
\operatorname{deg}_{S W}(\mathfrak{c}):=\mathcal{J}\left(\mathfrak{c}, \mathfrak{c}_{E}\right) \in \mathbb{Z} / p \mathbb{Z} .
$$

This depends only on the gauge equivalence class of $\mathfrak{c}$.

3.4. Periodic orbits and Seiberg-Witten solutions. Fix a class $\Gamma \in$ $H^{1}(M ; \mathbb{R})$. Let $\mathfrak{s}_{\Gamma}$ denote the $\operatorname{Spin}^{\mathbb{C}}$ structure whose spinor bundle splits as in (3.12) with the first Chern class of the bundle $E$ equal to the Poincaré dual of $\Gamma$. The theorem that follows refers to the versions of (3.13) as defined for this particular $\operatorname{Spin}^{\mathbb{C}}$ structure.

Theorem 3.1. Let $\boldsymbol{\mu}_{P}=\left\{\left(F, w_{F}\right), f, \Gamma, J\right\}$ and $\boldsymbol{\mu}_{S}=\left\{M, \mathfrak{s}_{\Gamma}, \varpi_{r}, g, \mathfrak{q}\right\}$ be corresponding periodic Floer homology and Seiberg-Witten-Floer cohomology data sets as in the statement of Theorem 1.1, and let $r \geq 1$. Use $\mathrm{e}^{r}$ to denote the space of gauge equivalence classes of solutions to the corresponding version of (3.13). Then:

(1) If $d_{\Gamma} \leq 0$ and $\Gamma \neq 0$, then $\mathrm{e}^{r}=\emptyset$ for all sufficiently large $r$.

(2) If $\Gamma=0$, then $\mathrm{e}^{r}$ consists of a single element for all sufficiently large $r$.

(3) Suppose that $d_{\Gamma}>0$. Let

$$
\mathfrak{X}= \begin{cases}\bigcup_{\Theta \in \mathcal{Z}} \mathfrak{C} \Theta & \text { if } \mathfrak{C} \Theta^{*}=\mathfrak{C} \Theta \text { for all } \Theta \in \mathcal{Z}, \\ \text { a finite subset of } \bigcup_{\Theta \in \mathcal{Z}} \mathfrak{C} \Theta^{*} & \text { otherwise. }\end{cases}
$$

There exists, for all sufficiently large $r$, a 1-1 map

$$
\Phi^{r}: \mathfrak{X} \rightarrow \mathfrak{C}^{r}
$$

with the following properties:

(a) The image of $\Phi^{r}$ consists of non-degenerate solutions to (3.13).

(b) If $x_{-}, x_{+} \in \mathfrak{X}$, then

$$
\operatorname{deg}_{S W}\left(\Phi^{r}\left(x_{+}\right)\right)-\operatorname{deg}_{S W}\left(\Phi^{r}\left(x_{-}\right)\right)=\operatorname{deg}_{\mathfrak{C Z}}\left(x_{-}\right)-\operatorname{deg}_{\mathfrak{C Z}}\left(x_{+}\right) .
$$

(c) If $\mathfrak{C} \Theta^{*}=\mathfrak{C} \Theta$ for all $\Theta \in \mathcal{Z}$ and $\mathfrak{X}=\bigcup_{\Theta \in \mathcal{Z}} \mathfrak{C} \Theta$, then $\Phi^{r}$ is also onto $\mathrm{e}^{r}$.

Proof of Theorem 3.1. There are five parts to the proof of this theorem. Part 1 constructs the solution whose existence is asserted by the second item. Part 2 constructs the map $\Phi^{r}$ from $\mathfrak{X}$ into $\mathcal{C}^{r}$ and proves that it is one to one. Part 3 proves items (3a) and (3b) of the assertions. Part 4 establishes some basic a priori bounds for solutions of (3.13). These bounds are then used in Part 5 to prove item (1), the uniqueness assertion of item (2), and item (3c). 
Part 1. The solution whose existence is asserted by item (2) can be constructed by copying almost verbatim what is done in the first parts of Section $2 \mathrm{~d}$ of [T9], with the following replacements:

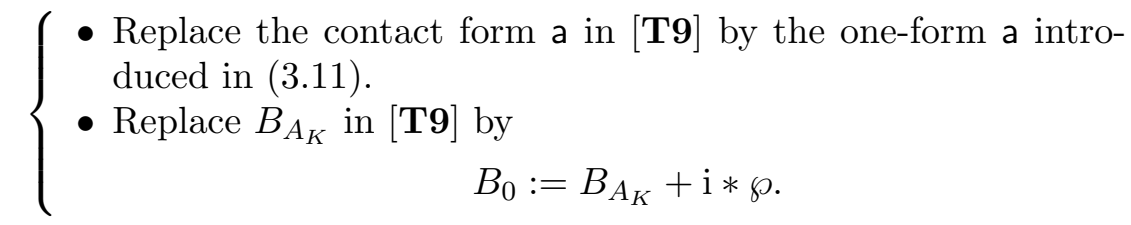

These replacements do not affect the arguments in Section 2d of [T9]; and these prove the following:

Lemma 3.4. Suppose that the $\operatorname{Spin}^{\mathbb{C}}$ structure is the canonical one. Then there exists a constant $\kappa>1$ such that for all $r \geq \kappa$, there is a solution, $\left(A_{0}, \psi_{0}\right)$, to (3.13) with the following properties:

- $\left|\psi_{0}\right| \geq 1-\kappa r^{-1 / 2}$ on the whole of $M$.

- If $(A, \psi)$ is a solution to (3.13) and if $|\psi| \geq 1-\kappa^{-1}$ on the whole of $M$, then $(A, \psi)$ is gauge equivalent to $\left(A_{0}, \psi_{0}\right)$.

Part 2. The map $\Phi^{r}$ is constructed by copying what is done in Section 3 of [T2]. The latter constructs the analog of the map $\Phi^{r}$ in the following context: First the orbits that appear in any given set from $\mathcal{Z}$ are closed integral curves of the Reeb vector field of a contact 1-from on $M$. Second, that same contact one-form is used as the one-form a to define (3.13). With the same sort of replacements as (3.16), these constructions can be applied in the present context with only cosmetic changes. The proof that the current version of $\Phi^{r}$ is injective is obtained by copying almost verbatim what is said in Section 3g of [T2] to prove the latter's Theorem 1.1. Note in this regard that the function $\mathrm{E}$ that appears in equation (1.12) and Theorem 1.1 of $[\mathbf{T 2}]$ has an analog here; the definition is the same:

$$
\mathrm{E}(A)=i \int_{M} \mathrm{a} \wedge * B_{A}
$$

What is denoted by $\ell_{\gamma}$ in [T2] corresponds here to $2 \pi q$, where $q$ is the period of $\gamma$. As a consequence,

$$
\sum_{(\gamma, m) \in \Theta} m \ell_{\gamma}=2 \pi d_{\Gamma}
$$

More is said in the upcoming Part 4 of the proof of Theorem 3.1 about the values of the function $\mathrm{E}$ in (3.17) on the space $\mathcal{C}^{r}$.

Part 3. What is asserted by items (3a) and (3b) of Theorem 3.1 has its analog in Theorem 1.1 of [T3]. Again, with the same sort of substitution 
as (3.16), the arguments in Section 2 of [T3] can be applied here with only cosmetic changes to prove items (3a) and (3b) of Theorem 3.1.

Part 4. This part of the proof establishes the key a priori bounds on the behavior of solutions to (3.15). The first of these is an analog of Lemma 2.3 in $[\mathbf{T} 4]$ :

Lemma 3.5. There exists a constant $\kappa>1$ with the following significance: Suppose that $r \geq \kappa$ and that $(A, \psi=(\alpha, \beta))$ is a solution to (3.13). Then:

(1) $|\alpha| \leq 1+\kappa r^{-1}$.

(2) $|\beta|^{2} \leq \kappa r^{-1}\left(1-|\alpha|^{2}\right)+\kappa^{2} r^{-2}$.

(3) $\left|\nabla_{A} \alpha\right|^{2} \leq \kappa r\left(1-|\alpha|^{2}\right)+\kappa^{2}$.

(4) $\left|\nabla_{A} \beta\right|^{2} \leq \kappa\left(1-|\alpha|^{2}\right)+\kappa^{2} r^{-1}$.

In addition, for each integer $q \geq 1$, there exists a constant $\kappa_{q}$ such that when $r \geq \kappa$, then

$$
\text { (5) }\left|\nabla_{A}^{q} \alpha\right|+r^{1 / 2}\left|\nabla_{A}^{q} \beta\right| \leq \kappa_{q} r^{q / 2} \text {. }
$$

Proof of Lemma 3.5. The first two item and the final item are proved by copying what is done in the proofs of Lemmas $2.2-2.4$ of [ $\mathbf{T} 7]$. The third and fourth items are proved by copying what is done in the proof of the analogous assertions of Lemma 2.3 in [T4].

The next a priori bound concerns the integral of $r\left(1-|\alpha|^{2}\right)$ over $M$. Note that a bound on this integral gives a bound on the value of the function $\mathrm{E}$ from (3.17). This follows by using (3.13) and the first two items in Lemma 3.5 to write

$$
* B_{A}=-\mathrm{i}\left(r\left(1-|\alpha|^{2}\right)+\mathfrak{e}_{1}\right) w_{f}+\mathfrak{e}_{2},
$$

where $\mathfrak{e}_{1}$ and $\mathfrak{e}_{2}$ are such that:

$$
\begin{aligned}
& \left|\mathfrak{e}_{1}\right| \leq c_{0}\left(1-|\alpha|^{2}\right)+c_{0}^{2} r^{-1} \\
& \left|\mathfrak{e}_{2}\right| \leq r^{1 / 2}\left|\left(1-|\alpha|^{2}\right)\right|+c_{0} \quad \text { and } \mathrm{a} \wedge \mathfrak{e}_{2}=0 .
\end{aligned}
$$

Lemma 3.6. There exists a constant $\kappa>1$ with the following significance: Suppose that $r \geq \kappa$ and that $(A, \psi)$ is a solution to (3.13). Then

$$
\left.r \int_{M}|1-| \alpha\right|^{2} \mid \leq \kappa
$$

Proof. Since $\frac{\mathrm{i}}{2 \pi} F_{A}$ represents the first Chern class of the bundle $E$ in de Rham cohomology, it follows that

$$
\mathrm{i} \int_{M} d t \wedge * B_{A}=4 \pi^{2} d_{\Gamma}
$$


Meanwhile, the first two items in Lemma 3.5 together with (3.18) imply that the integral on the left-hand side is no less than

$$
\left(1-c_{0}^{-1}\right) r \int_{M}\left(1-|\alpha|^{2}\right)-c_{0}
$$

This bounds the integral over $M$ of $r\left(1-|\alpha|^{2}\right)$ by an $r$ and $(A, \psi)$ independent constant. Together with the first item in Lemma 3.5, such a bound supplies the same sort of bound on the integral over $M$ of $\left|\left(1-|\alpha|^{2}\right)\right|$.

Part 5. This part proves assertions (1), (3c), and the uniqueness assertion in item (2) of Theorem 3.1. To start, consider a sequence $\left\{\left(r_{n},\left(A_{n}, \psi_{n}=\right.\right.\right.$ $\left.\left.\left.\left(\alpha_{n}, \beta_{n}\right)\right)\right)\right\}_{n=1,2, \ldots}$ such that $\left\{r_{n}\right\}_{n=1,2, \ldots}$ is unbounded, and such that $\left(A_{n}, \psi_{n}\right)$ obeys the $r=r_{n}$ version of (3.13). If $c_{1}(E)=0$, assume in addition that $\left(A_{n}, \psi_{n}\right)$ is not the solution $\left(A_{0}, \psi_{0}\right)$ given by Lemma 3.4.

Given the bound supplied by Lemma 3.6, the argument from Section $6 \mathrm{~d}$ of [T7] (or part I of [T6]) can be used with only notational modifications to find a finite set $\Theta=\{(\gamma, m)\}$ with the following properties:

- $\gamma$ is a closed integral curve of $\partial_{t}$ and $m$ is a positive integer.

- $\sum_{(\gamma, m) \in \Theta} m \gamma$ defines a chain that represents $\Gamma$ in $H_{1}(M ; \mathbb{Z})$.

- The loci $\alpha_{n}^{-1}(0)$ converges geometrically to $\sum_{(\gamma, m) \in \Theta} m \gamma$ in the following sense: Fix $\delta>0$. If $n$ is large, then $\left|\alpha_{n}\right| \geq 1-\delta$ at distances greater than $c_{0} r^{-1 / 2}$ from $\bigcup_{(\gamma, m) \in \Theta} \gamma$. Meanwhile, for any given $(\gamma, m) \in \Theta$, the restriction of $\alpha_{n} /\left|\alpha_{n}\right|$ to the boundary of the $\varphi_{\gamma}$ image of a disk $\{\tau\} \times D$ has degree $m$.

- $\frac{\mathrm{i}}{2 \pi} F_{A}$ converges as a current to $\sum_{(\gamma, m) \in \Theta} m \gamma$.

These last conclusions imply that $d_{\Gamma}>0$ if $\Theta \neq\{\emptyset\}$ and thus assertion (1) of Theorem 3.1. If $\Theta=\{\emptyset\}$, these conclusions with Lemma 3.4 imply the uniqueness assertion (2) of Theorem 3.1.

Granted $d_{\Gamma}>0$, then item (3c) of Theorem 3.1 is proved by copying almost verbatim the arguments that are given in Section $2 \mathrm{a}$ of [T4]. The latter prove an analog for the case when the one-form a in (3.13) is a contact one-form. This contact condition plays no essential role in the arguments in Section $2 \mathrm{a}$ of $[\mathbf{T 4}]$.

\section{Pseudoholomorphic curves and Seiberg-Witten solutions on $\mathbb{R} \times M$}

Fix a non-degenerate, area-preserving map $f: F \rightarrow F$ so as to construct the manifold $M$, and a monotone class $\Gamma \in H_{1}(M ; \mathbb{Z})$ with $d_{\Gamma}>0$. With $\Gamma$ given, define $\mathcal{Z}$ as in Section 3.2. Fix next a $J \in \mathcal{J}_{1 f}$. By Lemmas 2.12.4 , we may assume without loss of generality that the pair $(f, J)$ obeys the following constraint: Let $(\gamma, m)$ denote any given element from $\mathcal{Z}$ and let $q$ denote the period of $\gamma$. There is a disk $D \subset \mathbb{C}$ about the origin and a tubular 
neighborhood map $\varphi_{\gamma}: S^{1} \times D \rightarrow M$ such that:

- The curve $\gamma$ is $\varphi_{\gamma}(\cdot, 0)$.

- The vector field $q^{-1} \partial_{t}$ is the pushforward from $S^{1} \times D$ of

$$
\partial_{\tau}-2 \mathrm{i}\left(\nu_{\gamma} z+\mu_{\gamma} \bar{z}+\mathfrak{r}\right) \partial_{z}+2 \mathrm{i}\left(\nu_{\gamma} \bar{z}+\bar{\mu}_{\gamma} z+\overline{\mathfrak{r}}\right) \partial_{\bar{z}}
$$

where $\left(\nu_{\gamma}, \mu_{\gamma}\right)$ are given in (2.1) and where $\mathfrak{r}$ is zero on a neighborhood of $S^{1} \times\{0\}$.

- The almost complex structure $J$ is such that $J \cdot \varphi_{\gamma *} \partial_{z}=\varphi_{\gamma *} \mathrm{i} \partial_{z}$ on the image of a neighborhood of $S^{1} \times\{0\}$ under $\varphi_{\gamma}$.

Recall from Section 1.1 that $\mathcal{A} \subset \mathcal{Z}$ denotes the subset that consists of those elements $\Theta$ that do not partner hyperbolic integral curves of $\partial_{t}$ with numbers greater than 1. Given (4.1), the following is now a consequence of Lemma 3.2:

$$
\left\{\begin{array}{l}
\bullet \mathfrak{C} \Theta^{*}=\mathfrak{C} \Theta \text { for all } \Theta \in \mathcal{Z} \\
\bullet \mathfrak{C} \Theta \text { is a single point if } \Theta \in \mathcal{A}, \text { and } \mathfrak{C} \Theta=\emptyset \text { otherwise. }
\end{array}\right.
$$

Use the almost complex structure $J$ to define the metric on $M$ as done in the preceding section. Use the $\operatorname{Spin}^{\mathbb{C}}$-structure on $M$ whose spinor bundle splits as in (3.12) with $c_{1}(E)=e_{\Gamma}$. For $r \geq 1$, let $\mathcal{C}^{r}$ denote the corresponding space of gauge equivalence classes to (3.13). Because of (4.2), all sufficiently large $r$ versions of Theorem 3.1's map $\Phi^{r}$ supply a 1-1 correspondence between the set $\mathcal{A}$ and the set $\mathrm{e}^{r}$.

The body of this section concerns a map $\Psi^{r}$ that associates an instanton solution to versions of the Seiberg-Witten equations on $\mathbb{R} \times M$ to a weighted, pseudoholomorphic subvariety of the sort that is used to define the differential in periodic Floer homology. This map is described in the upcoming Section 4.3. The intervening sections set the stage for what is said in Section 4.3 about this map.

4.1. Pseudoholomorphic subvarieties in $\mathbb{R} \times M$. This subsection discusses certain issues concerning the differential for periodic Floer homology. To start, recall that the periodic Floer homology chain complex is the free $\mathbb{Z}$-module generated by equivalence classes of pairs of the form $(\Theta, \mathfrak{o})$ where $\Theta \in \mathcal{A}$ and $\mathfrak{o}$ is an ordering of the set of pairs in $\Theta$ of the form $(\gamma, 1)$ with $\gamma$ a hyperbolic Reeb orbit with even rotation number. The equivalence relation has $(\Theta, \mathfrak{o}) \sim(-1)^{\sigma}\left(\Theta, \mathfrak{o}^{\prime}\right)$ where $\sigma$ is 0 or 1 , which is the parity of the permutation that takes $\mathfrak{o}$ to $\mathfrak{o}^{\prime}$.

Let $\left(\Theta_{-}, \mathfrak{o}_{-}\right)$and $\left(\Theta_{+}, \mathfrak{o}_{+}\right)$denote generators of the periodic Floer chain complex. Hutchings observed that the extra data given by $\mathfrak{o}_{-}$and $\mathfrak{o}_{+}$can be used to associate a weight from the set $\{ \pm 1\}$ to each component of $\mathcal{M}_{1}\left(\Theta_{+}, \Theta_{-}\right)$; this is the weight $\sigma$ that is used to define via (1.9) the periodic Floer homology differential. The definition of this \pm 1 weight is identical in all respects to an analogous definition that is used to define embedded contact 
homology. The latter is given in Section 9 of [HT1] and also described in Section $3 \mathrm{~b}$ of $[\mathbf{T 3}]$. What follows immediately is a two part summary of how the \pm 1 weight is defined.

Part 1. Let $C \subset \mathbb{R} \times M$ denote an embedded, pseudoholomorphic curve. Then $C$ has a canonical complex structure, and $C$ 's normal bundle has a complex structure and, as a complex line bundle, a canonical holomorphic structure. Let $\mathrm{N}$ denote the latter bundle. Associated to $C$ is an operator $\mathcal{D}_{C}: C^{\infty}(C ; \mathrm{N}) \rightarrow C^{\infty}\left(C ; \mathrm{N} \otimes T^{0,1} C\right)$ depicted in (2.4). This operator defines a Fredholm operator from the space of $L_{1}^{2}$ sections along $C$ of $\mathrm{N}$ to the space of $L^{2}$ sections of $\mathrm{N} \otimes T^{0,1} C$. The assumption that $J \in \mathcal{J}_{1 f}$ guarantees that this Fredholm incarnation of (2.4) in the case when $C$ is not $\mathbb{R}$-invariant is surjective.

Suppose next that $\Theta \in \mathcal{A}$ and that $(\gamma, m) \in \Theta$. Associated to $\gamma$ is the $\mathbb{R}$-invariant, pseudoholomorphic cylinder $C=\mathbb{R} \times \gamma \subset \mathbb{R} \times M$. The vector field $\varphi_{\gamma_{*}} \partial_{z}$ along $\mathbb{R} \times \gamma$ identifies the normal bundle of $C$ with the product bundle $C \times \mathbb{C}$. Meanwhile, $\varphi_{\gamma}$ identifies $C$ with $\mathbb{R} \times S^{1}$. This understood, what is written in (2.4) can be viewed as a differential operator on $C^{\infty}(\mathbb{R} \times$ $\left.S^{1} ; \mathbb{C}\right)$. In this guise, the sections $\nu_{C}$ and $\mu_{C}$ become the functions $\nu_{\gamma}$ and $\mu_{\gamma}$ that appear in (4.1). What is written in (2.4) can be lifted to any given $p$-fold cover of $\mathbb{R} \times S^{1}$ and so act on $C^{\infty}(\mathbb{R} \times(\mathbb{R} /(2 \pi p \mathbb{Z})) ; \mathbb{C})$. Of interest are the cases when $p \leq m$. This lifted version of (2.4) defines a Fredholm operator mapping the space of $L_{1}^{2}$-functions on $\mathbb{R} \times(\mathbb{R} /(2 \pi p \mathbb{Z}))$ to the space of $L^{2}$-functions. This lifted version of $(2.4)$ is also denoted by $\mathcal{D}_{C}$ when $C=\mathbb{R} \times \gamma$. Note that such a lifted version of $\mathcal{D}_{C}$ has trivial kernel and cokernel when $\gamma$ is non-degenerate.

Part 2. Suppose that $\Theta_{-}$and $\Theta_{+}$come from $\mathcal{A}$. Recall from Section 2.1 that the assumption that $J \in \mathcal{J}_{1 f}$ implies the following:

- Each component of $\mathcal{M}_{1}\left(\Theta_{+}, \Theta_{-}\right)$is a smooth, one-dimensional manifold with a free $\mathbb{R}$-action that is induced by the action of $\mathbb{R}$ on $\mathbb{R} \times M$ as the constant translations along the $\mathbb{R}$ factor.

- Let $\Sigma \in \mathcal{M}_{1}\left(\Theta_{+}, \Theta_{-}\right)$. There is precisely one pair from $\Sigma$ whose pseudoholomorphic curve component is not $\mathbb{R}$-invariant.

- The latter pair has the form $(C, 1)$ with $C$ an embedded pseudoholomorphic subvariety which has no points in common with the $\mathbb{R}$-invariant subvarieties from $\Sigma$.

- The corresponding version of $\mathcal{D}_{C}$ has index 1 and trivial cokernel.

Granted the above, Quillen's ideas $[\mathbf{Q}]$ about determinant line bundles identify the real line

$$
\operatorname{det}\left(\oplus_{(C, m) \in \Sigma} \operatorname{Ker}\left(\mathcal{D}_{C}\right)\right)
$$

as the fiber over $\Sigma \in \mathcal{M}_{1}\left(\Theta_{+}, \Theta_{-}\right)$of the orientation sheaf, $\Lambda\left(\Theta_{+}, \Theta_{-}\right)$, of $\mathcal{M}_{1}\left(\Theta_{+}, \Theta_{-}\right)$. Definition 9.9 in $[$ HT1] can be used almost verbatim 
in the present context to define the notion of a coherent system of ori-

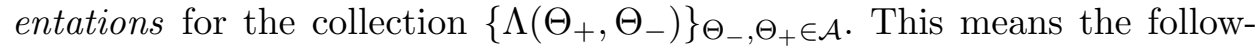
ing thing: Each $\Theta$ from $\mathcal{A}$ has a canonically associated $\mathbb{Z} / 2 \mathbb{Z}$-module, which we denote by $\Lambda(\Theta)$. Moreover, there is a canonical isomorphism from $\Lambda\left(\Theta_{+}, \Theta_{-}\right)$to $\Lambda\left(\Theta_{+}\right) \Lambda\left(\Theta_{-}\right)$. This understood, a set of orientations $\left\{\mathfrak{o}\left(\Theta_{+}, \Theta_{-}\right) \in \Lambda\left(\Theta_{+}, \Theta_{-}\right)\right\}_{\Theta_{-}, \Theta_{+} \in \mathcal{A}}$ is said to be coherent when there exists a corresponding set of orientations $\left\{\mathfrak{o}_{\Theta} \in \Lambda(\Theta)\right\}_{\Theta \in \mathcal{A}}$ such that any given $\mathfrak{o}\left(\Theta_{+}, \Theta_{-}\right)$is equal to $\mathfrak{o}\left(\Theta_{+}\right) \mathfrak{o}\left(\Theta_{-}\right)$.

Replace the phrase 'embedded contact homology' with 'periodic Floer homology' in Section 9.5 of [HT1] and Parts 3-6 in Section 3b of [T3] to see how a coherent system of orientations is canonically defined by assigning to each set $\Theta \in \mathcal{A}$ an ordering of the subset of pairs whose periodic orbit is hyperbolic and positive. Such orientation is denoted by $\mathfrak{o}_{p F h}$ in Theorem 4.1 below.

The orientation $\mathfrak{o}_{p F h}$ is used to define the \pm 1 contributions from each component of $\mathcal{M}_{1}\left(\Theta_{+}, \Theta_{-}\right)$as follows: The generator of the $\mathbb{R}$-action on $\mathcal{M}_{1}\left(\Theta_{+}, \Theta_{-}\right)$orients any given component. Either this orientation agrees with $\mathfrak{o}_{p F h}$ or not. If so, assign the component +1 , if not assign the component -1 .

4.2. Instantons on $\mathbb{R} \times \boldsymbol{M}$. With the class $\Gamma \in H_{1}(M ; \mathbb{Z})$ chosen, use $E$ in what follows to denote a complex line bundle over $M$ whose first Chern class is Poincaré dual to $\Gamma$. The $\operatorname{Spin}^{\mathbb{C}}$ structure used in what follows is such that its spinor bundle $\mathbb{S}$ splits as in (3.12).

Fix $r \geq 1$. The relevant versions of the Seiberg-Witten equations on $\mathbb{R} \times M$ constitute a system of equations for a pair $(A, \psi): \mathbb{R} \rightarrow \operatorname{Conn}(E) \times$ $C^{\infty}(M ; \mathbb{S}) ;$ these being

$$
\left\{\begin{array}{l}
\frac{\partial}{\partial s} A+B_{A}-r\left(\psi^{\dagger} \tau \psi-\mathrm{ia}\right)+\frac{1}{2} B_{0}=0, \\
\frac{\partial}{\partial s} \psi+D_{A} \psi=0,
\end{array}\right.
$$

where $B_{0}$ is as in (3.16).

Of interest, in what follows, are the instanton solutions; those whereby $\left.(A, \psi)\right|_{s}$ converges as $s \rightarrow \pm \infty$ to respective solutions to (3.13). The rest of this subsection concerns the solutions to (4.4). The discussion is in two parts.

Part 1. Fix an instanton solution to (4.4) and denote it by

$$
s \mapsto \mathfrak{d}(s)=\left.(A, \psi)\right|_{s} .
$$

Associated to $\mathfrak{d}$ is the differential operator on $C^{\infty}\left(\mathbb{R} \times M ; \mathrm{i} T^{*} M \oplus \mathbb{S} \oplus \mathrm{i} \mathbb{R}\right)$ that sends a section $(b, \eta, \phi)$ to the section with respective $\mathrm{i} T^{*} M, \mathbb{S}$ and $\mathrm{i} \mathbb{R}$ 
components

$$
\left\{\begin{array}{l}
\frac{\partial}{\partial s} b+* d b-d \phi-2^{-1 / 2} r^{1 / 2}\left(\psi^{\dagger} \tau \eta+\eta^{\dagger} \tau \psi\right), \\
\frac{\partial}{\partial s} \eta+D_{A} \eta+2^{1 / 2} r^{1 / 2}(\operatorname{cl}(b) \psi+\phi \psi) \\
\frac{\partial}{\partial s} \phi+* d * b-2^{-1 / 2} r^{1 / 2}\left(\eta^{\dagger} \psi-\psi^{\dagger} \eta\right) .
\end{array}\right.
$$

Because of items (3a) and (3c) of Theorem 3.1, this operator defines a Fredholm operator

$$
\mathfrak{D}_{\mathfrak{d}}: L_{1}^{2}\left(\mathbb{R} \times M ; \mathrm{i} T^{*} M \oplus \mathbb{S} \oplus \mathrm{i} \mathbb{R}\right) \rightarrow L^{2}\left(\mathbb{R} \times M ; \mathrm{i} T^{*} M \oplus \mathbb{S} \oplus \mathrm{i} \mathbb{R}\right) .
$$

The instanton $\mathfrak{d}$ is said to be non-degenerate when $\mathfrak{D}_{\mathfrak{d}}$ has trivial cokernel.

Fix a pair, $\mathfrak{c}_{-}$and $\mathfrak{c}_{+}$, of solutions to (3.13). Recall from Section 1.2 that $\mathcal{M}_{1}\left(\mathfrak{c}_{-}, \mathfrak{c}_{+}\right)$denotes the space of instanton solutions to (4.4) with the two properties: The limit as $s \rightarrow-\infty$ is $\mathfrak{c}_{-}$and whose limit as $s \rightarrow \infty$ is gauge equivalent to $\mathfrak{c}_{+}$. In addition, the corresponding version of (4.5) has Fredholm index 1 . Note that the space $\mathcal{M}_{1}\left(\mathfrak{c}_{-}, \mathfrak{c}_{+}\right)$is canonically associated to the pair of respective gauge equivalence classes of $\mathfrak{c}_{-}$and $\mathfrak{c}_{+}$. This is because there is a canonical identification between $\mathcal{M}_{1}\left(\mathfrak{c}_{-}, \mathfrak{c}_{+}\right)$and $\mathcal{M}_{1}\left(u \mathfrak{c}_{-}, \mathfrak{c}_{+}\right)$for any given map $u: M \rightarrow S^{1}$, sending a given instanton $\mathfrak{d}=(A, \psi)$ to the instanton $u \cdot \mathfrak{d}=\left(A-u^{-1} d u, u \psi\right)$.

The space $\mathcal{M}_{1}\left(\mathfrak{c}_{-}, \mathfrak{c}_{+}\right)$has the structure of a smooth, one-dimensional manifold in a neighborhood of any non-degenerate instanton. This space also enjoys a free $\mathbb{R}$-action, this induced from the action of $\mathbb{R}$ on $\mathbb{R} \times M$ by translation along the $\mathbb{R}$ factor. As the assignment $\mathfrak{d} \rightarrow \mathfrak{D}_{\mathfrak{d}}$ is $\mathbb{R}$-equivariant, so it follows that a component in $\mathcal{M}_{1}\left(\mathfrak{c}_{-}, \mathfrak{c}_{+}\right)$of a non-degenerate instanton consists solely of non-degenerate instantons. In particular, such a component is a smooth manifold with a diffeomorphism to $\mathbb{R}$ that intertwines the aforementioned $\mathbb{R}$-action with the translation action of $\mathbb{R}$ on itself.

Part 2. Let $\mathfrak{c}_{-}$and $\mathfrak{c}_{+}$for the moment denote a given pair of non-degenerate elements in $\operatorname{Conn}(E) \times C^{\infty}(M ; \mathbb{S})$. Reintroduce the notation $\mathfrak{P}=\mathfrak{P}\left(\mathfrak{c}_{-}, \mathfrak{c}_{+}\right)$ from Section 1.2, now regarded as a space of piecewise differentiable maps from $\mathbb{R}$ to $\operatorname{Conn}(E) \times C^{\infty}(M ; \mathbb{S})$. Each $\mathfrak{d} \in \mathfrak{P}$ has its corresponding version of (4.5). Given that both $\mathfrak{c}_{-}$and $\mathfrak{c}_{+}$are non-degenerate, $\mathfrak{d}$ 's version of (4.5) defines a Fredholm operator, $\mathfrak{D}_{\mathfrak{d}}$, mapping the space of $L_{1}^{2}$ sections over $\mathbb{R} \times M$ of the bundle $\mathrm{i} T^{*} M \oplus \mathbb{S} \oplus \mathrm{i} \mathbb{R}$ to the space of $L^{2}$ sections of this same bundle. Quillen $[\mathbf{Q}]$ showed (in a somewhat different context) how such operators define a real line bundle, $\operatorname{det}\left(\mathfrak{D}_{\mathfrak{d}}\right) \rightarrow \mathfrak{P}$. In particular, if $\mathfrak{d} \in \mathfrak{P}$ is such that either the kernel or cokernel of $\mathfrak{D}_{\mathfrak{d}}$ is non-trivial, then the fiber of $\operatorname{det}\left(\mathfrak{D}_{\mathfrak{d}}\right)$ at a given $\mathfrak{d} \in \mathfrak{P}$ has a canonical identification with $\bigwedge^{\max }\left(\operatorname{Ker}\left(\mathfrak{D}_{\mathfrak{d}}\right)\right) \times_{\mathbb{R}}\left(\bigwedge^{\max } \operatorname{Coker}\left(\mathfrak{D}_{\mathfrak{d}}\right)\right)$. Introduce $\Lambda\left(\mathfrak{c}_{-}, \mathfrak{c}_{+}\right)$to denote the orientation sheaf of $\operatorname{det}(\mathfrak{D})$. 
Take $r \gg 1$ so that Theorem 3.1 can be used to conclude that all solutions to (3.13) are non-degenerate. Granted this non-degeneracy, Chapter 20 of $[\mathbf{K M}]$ associates to each gauge equivalence class $\mathfrak{c} \in \mathrm{C}^{r}$ an associated $\mathbb{Z} / 2 \mathbb{Z}$ module, $\Lambda(\mathfrak{c})$, with the following property: If $\mathfrak{c}_{-}$and $\mathfrak{c}_{+}$are any two solutions to (3.13), then there is a canonical isomorphism between the modules $\Lambda\left(\mathfrak{c}_{-}\right) \otimes_{\mathbb{Z} / 2 \mathbb{Z}} \Lambda\left(\mathfrak{c}_{+}\right)$and $\Lambda\left(\mathfrak{c}_{-}, \mathfrak{c}_{+}\right)$. In particular, a choice of $\mathfrak{o}\left(\mathfrak{c}_{-}\right) \in \Lambda\left(\mathfrak{c}_{-}\right)$ and $\mathfrak{o}\left(\mathfrak{c}_{+}\right) \in \Lambda\left(\mathfrak{c}_{+}\right)$defines a unique element in $\Lambda\left(\mathfrak{c}_{-}, \mathfrak{c}_{+}\right)$. A collection of orientations $\left\{\mathfrak{o}\left(\mathfrak{c}_{-}, \mathfrak{c}_{+}\right) \in \Lambda\left(\mathfrak{c}_{-}, \mathfrak{c}_{+}\right)\right\}_{\mathfrak{c}_{-}, \mathfrak{c}_{+} \in \mathrm{e}^{r}}$ is said to be coherent if there exists a corresponding set of orientations $\{\mathfrak{o}(\mathfrak{c}) \in \Lambda(\mathfrak{c})\}$ such that any given $\mathfrak{o}\left(\mathfrak{c}_{-}, \mathfrak{c}_{+}\right)$is equal to $\mathfrak{o}\left(\mathfrak{c}_{-}\right) \mathfrak{o}\left(\mathfrak{c}_{+}\right)$.

The relevance of this orientation business to Seiberg-Witten-Floer cohomology is as follows: Suppose that $\mathfrak{c}_{-}$and $\mathfrak{c}_{+}$are solutions to (3.13), and suppose that $\mathfrak{d} \in \mathcal{M}_{1}\left(\mathfrak{c}_{-}, \mathfrak{c}_{+}\right)$is non-degenerate. Then the restriction of $\Lambda\left(\mathfrak{c}_{-}, \mathfrak{c}_{+}\right)$to the component of $\mathcal{M}_{1}\left(\mathfrak{c}_{-}, \mathfrak{c}_{+}\right)$containing $\mathfrak{d}$ is canonically isomorphic to the latter's orientation sheaf. With this understood, fix orientations $\{\mathfrak{o}(\mathfrak{c}) \in \Lambda(\mathfrak{c})\}_{\mathfrak{c} \in \mathrm{e}^{r}}$ so as to define a collection of coherent orientations for

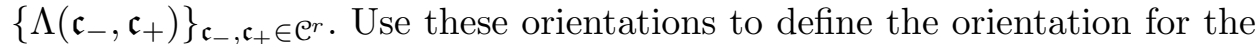

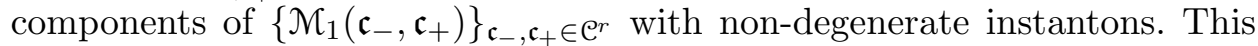
orientation is denoted in what follows by $\mathfrak{o}_{S W}$. Let $\mathcal{M} \subset \mathcal{M}_{1}\left(\mathfrak{c}_{-}, \mathfrak{c}_{+}\right)$denote a component with non-degenerate instantons. The generator of the $\mathbb{R}$-action on $\mathcal{M}$ also orients $\mathcal{M}$. This orientation is denoted by $\mathfrak{o}_{\mathbb{R}}$. Now view $\mathfrak{c}_{-}$and $\mathfrak{c}_{+}$as generators of the Seiberg-Witten-Floer cohomology complex. Then $\mathcal{M}$ contributes +1 to the sum that defines via (1.16) the multiple of $\mathfrak{c}_{-}$in the coboundary of $\mathfrak{c}_{+}$when $\mathfrak{o}_{S W}=\mathfrak{o}_{\mathbb{R}}$. Otherwise, $\mathcal{M}$ contributes -1 .

4.3. Pseudoholomorphic curves and instantons. Assume as usual that $\Gamma$ is monotone. Therefore, both $\mathcal{M}_{1}\left(\Theta_{+}, \Theta_{-}\right)$and $\mathcal{M}_{1}\left(\mathfrak{c}_{-}, \mathfrak{c}_{+}\right)$have finitely many components. Moreover,

$$
\left[w_{f}\right]=\kappa c_{1}(\mathfrak{s}) \quad \text { for some } \kappa \in \mathbb{R}
$$

because $\Gamma$ is monotone, and (6.4) with (3.15) imply the following: Given any $\mathfrak{d}(s)=(A, \psi) \in \mathfrak{P}\left(\mathfrak{c}_{-}, \mathfrak{c}_{+}\right)$for a fixed pair $\mathfrak{c}_{-}, \mathfrak{c}_{+}$, a bound on its spectral flow $\mathcal{J}(\mathfrak{d})$ gives rise to a bound on the integral

$$
\frac{\mathrm{i}}{2 \pi} \int_{\mathbb{R} \times M} \frac{\partial A}{\partial s} d s \wedge w_{f}
$$

With this said, the following is Section 4's main theorem.

Theorem 4.1. Let $\boldsymbol{\mu}_{P}=\left\{\left(F, w_{F}\right), f, \Gamma, J\right\}$ and $\boldsymbol{\mu}_{S}=\left\{M, \mathfrak{s}_{\Gamma}, \varpi_{r}, g, \mathfrak{q}\right\}$ be as in the statement of Theorem 1.1. Then there exists a constant $\kappa>1$, and given $N>\kappa, \delta<\kappa^{-1}$ and a $(\delta, N)$-approximation $\left(f^{\prime}, J^{\prime}\right)$ to $(f, J)$, there exists $\kappa^{\prime} \geq \kappa$ such that if $r \geq \kappa^{\prime}$, then the following is true: Use $\left(f^{\prime}, J^{\prime}\right)$ to 
define $\mathcal{A}$ and $\mathrm{e}^{r}$.

(0) Theorem 3.1 can be invoked so as to provide the identification, $\Phi^{r}$, between $\mathcal{A}$ and $\mathrm{e}^{r}$.

(1) Fix a pair $\Theta_{-}$and $\Theta_{+}$in $\mathcal{A}$; and let $\mathfrak{c}_{-}$and $\mathfrak{c}_{+}$denote solutions to (3.13) whose respective gauge equivalence classes are $\Phi^{r}\left(\Theta_{-}\right)$and $\Phi^{r}\left(\Theta_{+}\right)$. Let $\mathcal{M}_{1}\left(\Theta_{+}, \Theta_{-}\right)$and $\mathcal{M}_{1}\left(\mathfrak{c}_{-}, \mathfrak{c}_{+}\right)$be the versions defined using $\left(f^{\prime}, J^{\prime}\right)$.

(a) The space $\mathcal{M}_{1}\left(\mathfrak{c}_{-}, \mathfrak{c}_{+}\right)$has a finite set of components and each component consists of non-degenerate solutions to (4.4).

(b) There is a smooth $\mathbb{R}$-equivariant diffeomorphism

$$
\Psi^{r}: \mathcal{M}_{1}\left(\Theta_{+}, \Theta_{-}\right) \rightarrow \mathcal{M}_{1}\left(\mathfrak{c}_{-}, \mathfrak{c}_{+}\right) .
$$

(2) There are coherent systems of orientations for sheaves

$\left\{\Lambda\left(\Theta_{+}, \Theta_{-}\right)\right\}_{\Theta_{-}, \Theta_{+} \in \mathcal{A}}$ and $\left\{\Lambda\left(\mathfrak{c}_{-}, \mathfrak{c}_{+}\right)\right\}_{\mathfrak{c}_{-}, \mathfrak{c}_{+} \in \mathrm{C}^{r}}$ such that any given $\left(\Theta_{-}, \Theta_{+}\right)$version of $\Psi^{r}$ maps the orientation $\mathfrak{o}_{p F h}$ to the orientation $\mathfrak{o}_{S W}$.

The proof of this theorem is given below modulo certain facts about solutions of (4.4) that are derived in the upcoming Section 5.

Proof of Theorem 4.1, Part 1. The $\mathbb{R}$-equivariant map

$$
\Psi^{r}: \mathcal{M}_{1}\left(\Theta_{+}, \Theta_{-}\right) \rightarrow \mathcal{M}_{1}\left(\mathfrak{c}_{-}, \mathfrak{c}_{+}\right)
$$

is constructed by copying the construction given in Sections 4-7 of [T2]. The latter sections of [T2] construct an analogous map for the versions of (3.13) and (4.4) that take the one-form a to be a contact one-form. The fact that the [T2] versions of (3.13) and (4.4) use a contact one-form does not play a role in the constructions. By the same token, the arguments from Section $7 \mathrm{k}$ of $[\mathbf{T 2}]$ can be borrowed almost verbatim to prove that $\Psi^{r}$ is injective when $r$ is large.

The assertion that $\Psi^{r}$ maps $\mathcal{M}_{1}\left(\Theta_{+}, \Theta_{-}\right)$onto a union of components of $\mathcal{M}_{1}\left(\mathfrak{c}_{-}, \mathfrak{c}_{+}\right)$that contain solely non-degenerate instantons can be had by copying the arguments given in Section 3a of [T3] that prove the analogous assertion for when (3.17) and (4.4) take the one-form a to be a contact form. Assertion (2) of Theorem 4.1 are proved using almost verbatim the arguments from Section $3 \mathrm{~b}$ of [T3] that prove the analog for the case when the one-form a is a contact one-form.

Proof of Theorem 4.1. Part 2. It remains only to prove that $\Psi^{r}$ maps $\mathcal{M}_{1}\left(\Theta_{+}, \Theta_{-}\right)$onto $\mathcal{M}_{1}\left(\mathfrak{c}_{-}, \mathfrak{c}_{+}\right)$. The arguments for this borrow heavily from what is done in Sections 3-7 of [T4] to prove an analogous result in the case when the one-form a that appears in (3.13) and (4.4) is a contact form. As in [T4], the proof that $\Psi^{r}$ maps $\mathcal{M}_{1}\left(\Theta_{+}, \Theta_{-}\right)$onto $\mathcal{M}_{1}\left(\mathfrak{c}_{-}, \mathfrak{c}_{+}\right)$has three distinct parts which, for lack of better terms, will be called estimation, convergence, and perturbation. 
(a) The estimation part. Here and in [T4], the global part of the proof establishes certain properties of instanton solutions to the respective large $r$ versions of (4.4). In [T4], the properties in question are summarized by the various results in Section 3 of [T4] and in Lemmas 5.2 and 5.3 of [T4]. Each of the relevant results in $[\mathbf{T 4}]$ has an analog here. Corresponding results are as follows: Lemmas 3.1, 3.6, 3.8, 3.9 and 3.11 in [T4] supply pointwise a priori bounds for instanton solutions. These lemmae, respectively, correspond to the upcoming Lemmas 5.2, 5.6, 5.10, 5.11 and 5.12. The pointwise bounds given by equation (3.35) from [T4] correspond to those given in the upcoming equation (5.47). Lemma 3.10 in [T4] asserts a crucial monotonicity result; its analog here is Lemma 5.9. Lemmas 5.2 and 5.3 in [T4] supply crucial a priori bounds for a certain integral; Lemma 5.8 below plays a similar role.

Note however that the statements of Lemmas 5.3-5.10 and Lemma 5.12 below differ from their analogs in [T4] in the following aspect: They share the common condition (5.8), which asks for a bound on the integral (4.7), while in $[\mathbf{T 4}]$, this condition is substituted by the requirement that the spectral flow of the instanton is bounded. However, as observed in the beginning of this subsection, the former condition implies the latter when (6.4) holds. This is true both in the monotone case under discussion, and in the context of $[\mathbf{T 4}]$, where $w_{f}$ is replaced by $d$ a.

(b) The convergence part. This part uses the global results to assign an element in $\mathcal{M}_{1}\left(\Theta_{+}, \Theta_{-}\right)$to a given instanton in $\mathcal{M}_{1}\left(\mathfrak{c}_{-}, \mathfrak{c}_{+}\right)$. The arguments here borrow heavily from what is done in Sections $5-7$ in the article $\mathrm{SW} \Rightarrow \mathrm{Gr}$ from [T6]. There is necessarily more, in order to deal with the fact that $\mathbb{R} \times M$ is non-compact. In any event, this part of the argument in [T4] occupies the latter's Section 4 with the results summarized by Proposition 4.1 in [T4]. In Lemma 5.8 below, we show that the condition of Proposition 4.1 in [T4] is met in our case, and this replaces Proposition 5.1 in [T4]. The rest of the convergence part of the proof here can be copied from Section 4 of [T4] with only cosmetic and notational changes. This understood, no more will be said here about this part of the proof.

(c) The perturbation part. This part of the proof establishes that the correspondence that is established in the convergence part of the proof is given by the map $\Psi^{r}$. This is to say the following: Let $\mathfrak{d} \in \mathcal{M}_{1}\left(\mathfrak{c}_{-}, \mathfrak{c}_{+}\right)$and let $\Sigma \in \mathcal{M}_{1}\left(\Theta_{+}, \Theta_{-}\right)$denote its partner from part (b) above. Then $\mathfrak{d}$ is the image via $\Psi^{r}$ of $\Sigma$. This part of the proof in [T4] occupies the latter's Sections 6 and 7. This part of the proof also calls on the global results mentioned above. The analogous perturbation part of the proof here can be copied from Sections 6 and 7 of [T4], again with only cosmetic and notational changes. As a consequence, no more will be said about this part of the proof. 
4.4. The proof of Theorem 1.1. The periodic Floer homology is defined once given a pair $(f, J)$ with $f: F \rightarrow F$ an area-preserving diffeomorphism and with $J \in \mathcal{J}_{1 f}$. With this pair chosen, the periodic Floer homology is defined for a chosen monotone class $\Gamma \in H_{1}(M ; \mathbb{Z})$. The assertions in Theorem 1.1 for the cases $d_{\Gamma}<0$ and $d_{\Gamma}=0$ follow directly from assertions (1) and (2) in Theorem 3.1, respectively. This understood, assume henceforth that $d_{\Gamma}>0$.

Fix some very large integer $N$, chosen so that $d_{\Gamma} \ll N$. This guarantees the following: Reintroduce the set $\mathcal{Z}$ from Section 3.2, whose typical element, $\Theta$, consists of pairs of the form $(\gamma, m)$ with $\gamma$ a periodic orbit and $m$ a positive integer. Require in addition that no two pairs from $\Theta$ have the same periodic orbit, and that $\sum_{(\gamma, m) \in \Theta} m \gamma$ represent the class $\Gamma$. Let $\Theta \in \mathcal{Z}$ and let $(\gamma, m) \in \Theta$. Then $\gamma$ has period much less than $N$. Now fix a small enough $\delta>0$ to invoke Proposition 2.1. This proposition finds a $(\delta, N)$ approximation $\left(f^{\prime}, J^{\prime}\right)$ to the original pair $(f, J)$. As Proposition 2.1 finds a canonical isomorphism between the respective $(f, J)$ and $\left(f^{\prime}, J^{\prime}\right)$ versions of periodic Floer homology, it is sufficient to suppose at the outset that the pair $(f, J)$ is such that the conditions in (4.1) hold. This is assumed in what follows.

For each $\Theta \in \mathcal{Z}$, introduce as in Section 3.2 the sets $\mathfrak{C} \Theta$ and $\mathfrak{C} \Theta^{*}$. It is a consequence of Lemma 3.2 that these two sets are the same. Moreover, $\mathfrak{X}=\bigcup_{\Theta \in \mathcal{Z}} \mathfrak{C} \Theta$ has a canonical identification with the set $\mathcal{A}$ that generates the periodic Floer homology chain complex. This understood, then all $r \geq c_{0}$ versions of the map $\Phi^{r}$ from Theorem 3.1 supply an identification between the set $\mathcal{A}$ and the set $\mathrm{C}^{r}$ of gauge equivalence classes of solutions to (3.13). Theorem 3.1(3a) asserts that each equivalence class in $\mathrm{e}^{r}$ consists of nondegenerate solutions to (3.13). As a consequence, the set $\mathrm{C}^{r}$ provides a set of generators for the Seiberg-Witten-Floer cohomology cochain complex. Note in this regard that both $\mathcal{A}$ and $\mathrm{e}^{r}$ are finite sets. Recall that $p \neq 0$ denote the divisibility of $c_{\Gamma}=c_{1}\left(\mathfrak{s}_{\Gamma}\right)$ in $H^{2}(M ; \mathbb{Z}) /$ Tors. Since by assumption, $(f, J)$ is of the modified form described in Sections 2.1, 2.2, we may invoke Lemma 3.3 which asserts that with this choice, $\operatorname{deg}_{p F h}(\Theta)=\operatorname{deg}_{\mathfrak{C Z}}(x)$. Thus, by Theorem $3.1(3 \mathrm{~b})$, the map $\Phi^{r}$ reverses the relative $\mathbb{Z} / p \mathbb{Z}$-grading of these generators. The $\mathbb{Z}$-linear extension of Theorem 3.1's identification from the generators to the respective chain and cochain complexes defines an isomorphism between these complexes.

Consider now the ramifications of Theorem 4.1. Let $\Theta_{-}$and $\Theta_{+}$denote any given pair from $\mathcal{A}$, and let $\mathfrak{c}_{-}=\Phi^{r}\left(\Theta_{-}\right)$and $\mathfrak{c}_{+}=\Phi^{r}\left(\Theta_{+}\right)$denote solutions to (3.13) as described in Theorem 3.1. According to assertion (1a) of Theorem 4.1, each component of $\mathcal{M}_{1}\left(\mathfrak{c}_{-}, \mathfrak{c}_{+}\right)$is non-degenerate. As a consequence, the collection $\left\{\mathcal{M}_{1}\left(\mathfrak{c}_{-}, \mathfrak{c}_{+}\right)\right\}_{\mathfrak{c}_{-}, \mathfrak{c}_{+} \in \mathcal{C}^{r}}$ can be used to define the differential on the Seiberg-Witten-Floer cochain complex via the formula (1.16). 
Let $\Theta_{-}, \Theta_{+}$and $\mathfrak{c}_{-}, \mathfrak{c}_{+}$be as the above. Then assertion (1b) of Theorem 4.1 supplies an $\mathbb{R}$-equivariant diffeomorphism between $\mathcal{M}_{1}\left(\Theta_{+}, \Theta_{-}\right)$ and $\mathcal{M}_{1}\left(\mathfrak{c}_{-}, \mathfrak{c}_{+}\right)$. According to assertion (2) of Theorem 4.1, this diffeomorphism can be assumed to preserve the respective coherent orientations. This implies that the integer $\sigma\left(\Theta_{+}, \Theta_{-}\right)$that is used in (1.9) to define the periodic Floer homology differential is identical to the corresponding integer $\sigma\left(\mathfrak{c}_{-}, \mathfrak{c}_{+}\right)$ that is used in (1.16) to define the Seiberg-Witten-Floer cohomology differential. It follows directly from this that the identification between the respective generating sets given by Theorem 3.1 intertwines the action on these generators of the respective differentials. As a consequence, the isomorphism provided by Theorem 3.1 between the respective chain and cochain complexes descends to give a relative $\mathbb{Z} / p \mathbb{Z}$-grading reversing isomorphism between the periodic Floer homology and the Seiberg-Witten-Floer cohomology. This is the isomorphism asserted in Theorem 1.1.

\section{The global arguments: properties of instantons}

This section derives the properties of solutions to (4.4) that constitute the global part of Part 2 of the proof of Theorem 4.1. As noted in the preceding subsection, everything done here has an analog in either Section 3 or Section 5 of [T4]. In addition, proofs of analogous assertions are very similar; and in some cases the similarity is such as to warrant directing the reader to the corresponding [T4] argument.

5.1. Special functions on $\operatorname{Conn}(E) \times C^{\infty}(M ; \mathbb{S})$. This first subsection introduces some functions of pairs $(A, \psi)$ that play a central role in the proof of Theorem 4.1. To this end, reintroduce the reference Hermitian connection $A_{E}$ on $E$ that was used in Section 3.3 to define the degree of a non-degenerate element in $\operatorname{Conn}(E) \times C^{\infty}(M ; \mathbb{S})$. This done, write $A=A_{E}+\hat{\mathrm{a}}_{A}$ with $\hat{\mathrm{a}}_{A} \in$ $\Omega^{1}(M ; \mathbb{R})$. This done, introduce the following important functionals:

$$
\begin{aligned}
\mathfrak{c}(A) & =-\int_{M} \hat{\mathrm{a}}_{A} \wedge d \hat{\mathrm{a}}_{A}-2 \int_{M} \hat{\mathrm{a}}_{A} \wedge *\left(B_{E}+\frac{1}{2} B_{A_{K}}\right) ; \\
Q_{F}(A) & =\mathrm{i} \int_{M} \hat{\mathrm{a}}_{A} \wedge w_{f} ; \\
\mathfrak{a}(A, \psi) & =\frac{1}{2} \mathfrak{c} \mathfrak{s}-\frac{\mathrm{i}}{2} \int_{M} \hat{\mathrm{a}}_{A} \wedge \varpi_{r}+r \int_{M} \psi^{\dagger} D_{A} \psi .
\end{aligned}
$$

The first functional above is the Chern-Simons functional, and the third is the action functional in the version of Seiberg-Witten-Floer theory relevant to us: Any given pair $(A, \psi) \in \operatorname{Conn}(E) \times C^{\infty}(M ; \mathbb{S})$ is a solution to (3.13) if and only if $(A, \psi)$ is a critical point of $\mathfrak{a}$. Meanwhile, (4.4) are the formal (downward) gradient flow equations for $\mathfrak{a}$ when the latter's gradient is defined using the $L^{2}$ inner product on the tangent space to $\operatorname{Conn}(E) \times C^{\infty}(M ; \mathbb{S})$. 
In general, none of these functionals are fully gauge-invariant. They vary with the cohomology class of the gauge transformation as follows:

$$
\begin{aligned}
\mathfrak{c} \mathfrak{s}\left(A-u^{-1} d u\right)-\mathfrak{c s}(A) & =\left\langle 4 \pi^{2} c_{1}(\mathfrak{s})[u]\right\rangle, \\
Q_{F}\left(A-u^{-1} d u\right)-Q_{F}(A) & =\left\langle 2 \pi\left[w_{f}\right],[u]\right\rangle \\
\mathfrak{a}\left(A-u^{-1} d u\right)-\mathfrak{a}(A) & =-\left\langle 2 \pi r\left[w_{f}\right],[u]\right\rangle .
\end{aligned}
$$

However, given two configurations $\mathfrak{c}_{-}, \mathfrak{c}_{+}$, and an element $\mathfrak{d}(s) \in \mathfrak{P}\left(\mathfrak{c}_{-}, \mathfrak{c}_{+}\right)$ from $u_{-} \cdot \mathfrak{c}_{-}$to $u_{+} \cdot \mathfrak{c}_{+}$, where $u_{-}, u_{+} \in C^{\infty}(M ; U(1))$, the value

$$
\Delta \mathfrak{a}(\mathfrak{d}):=\mathfrak{a}\left(u_{+} \cdot \mathfrak{c}_{+}\right)-\mathfrak{a}\left(u_{-} \cdot \mathfrak{c}_{-}\right)
$$

depends only on the gauge equivalence classes of $\mathfrak{c}_{-}, \mathfrak{c}_{+}$, and the relative homotopy class of $\mathfrak{d}(s)$. Thus, similarly to the grading function $\mathfrak{J}\left(\mathfrak{c}_{-}, \mathfrak{c}_{+} ; \mathfrak{h}\right)$ introduced in Section 1.2, we shall write

$$
\Delta \mathfrak{a}\left(\mathfrak{c}_{-}, \mathfrak{c}_{+} ; \mathfrak{h}\right)=\Delta \mathfrak{a}(\mathfrak{d})
$$

for any $\mathfrak{d}(s) \in \mathfrak{P}\left(\mathfrak{c}_{-}, \mathfrak{c}_{+}\right)$in the relative homotopy class $\mathfrak{h}$. Use the same notation convention for the functionals $\mathfrak{c s}$ and $Q_{F}$ as well. Note that $\Delta Q_{F}$ is $2 \pi$ times the integral (4.7). The lemma that follows discusses the values of these functions for solutions of the Seiberg-Witten equations (3.13).

Lemma 5.1. There exists $\kappa>1$ with the following significance: Suppose that $r \geq \kappa$ and that $\mathfrak{c}=(A, \psi)$ is a solution to (3.13) on $M$. Then there exists a gauge transformation $u \in C^{\infty}(M ; U(1))$ such that

$$
\begin{aligned}
|\mathfrak{c s}(u \cdot \mathfrak{c})| & \leq \kappa r^{1 / 2}, \\
\left|Q_{F}(u \cdot \mathfrak{c})\right| & \leq \kappa, \\
|\mathfrak{a}(u \cdot \mathfrak{c})| & \leq \kappa r .
\end{aligned}
$$

Proof of Lemma 5.1. First, note that by (3.18) and the first two items in Lemma 3.5 , we have the pointwise bound

$$
\left|F_{A}\right| \leq c_{0} r
$$

Choose to work in a gauge

$$
d^{*} \hat{\mathrm{a}}_{A}=0 .
$$

In this gauge, we may write

$$
\hat{\mathrm{a}}_{A}=a^{\prime}+a_{h},
$$

where $a^{\prime}$ is coexact and $a_{h}$ is harmonic. By performing a further gauge transformation, we may require that

$$
\left|a_{h}\right|^{2} \leq b^{1}(M) \text {. }
$$

On the other hand, the above pointwise bound on $F_{A}$ implies via a standard rescaling and elliptic regularity argument (see, e.g., the proof of Lemma 5.6 below) that we have

$$
\left|a^{\prime}\right| \leq c_{0} r^{1 / 2}
$$


Thus, by Lemma 3.6, with this choice of gauge

$$
\mathfrak{c} \mathfrak{s}(A) \leq c_{0}\left(\left|\hat{\mathrm{a}}_{A}\right|+1\right)\left(\int_{M}\left|F_{A}\right|+1\right) \leq c_{0}^{\prime} r^{1 / 2} .
$$

Consider next the bound for $\left|Q_{F}\right|$. To this end, fix $(\gamma, m) \in \Theta$ and use the fact that $H^{2}\left(S^{1} \times D ; \mathbb{R}\right)=0$ to find a smooth one-form $y_{\gamma}$ with support on the image of $\varphi_{\gamma}$ and such that $w_{f}-d y_{\gamma}=0$ on the $\varphi_{\gamma}$ image of the points in $S^{1} \times D$ with distance $c_{0}^{-1}$ from $S^{1} \times\{0\}$. On the other hand, one may pointwisely estimate $\left|a^{\prime}\right|$ by multiplying the Green's function on $F_{A}-F_{0}$ and integrate over $M$. Together with Lemmas 3.5, 3.6, and the Seiberg-Witten equations (3.13), this shows that $\left|a^{\prime}\right| \leq c_{0}$ outside the images of $\varphi_{\gamma}$ 's.

Set

$$
w_{f}^{\prime}=w_{f}-\sum_{(\gamma, m) \in \Theta} d y_{\gamma}
$$

Use this two-form to write

$$
Q_{F}(A)=i \int_{M} \hat{\mathrm{a}}_{A} \wedge w_{f}^{\prime}+i \sum_{(\gamma, m) \in \Theta} \int_{M} F_{A} \wedge y_{\gamma}
$$

and examines the two terms on the right-hand side separately.

The integrand in the first term above is supported away from the images of $\varphi_{\gamma}$ 's, where $|\hat{a}|<c_{0}$ with our choice of gauge, as we have just seen. This shows that the first term is bounded by a constant.

Meanwhile, the second term is also bounded by a constant, again by combining Lemmas 3.5, 3.6, and the Seiberg-Witten equations (3.13).

Lastly, to bound $\mathfrak{a}$, note that by $(3.13), \int_{M} \psi^{\dagger} D_{A} \psi=0$. This said, the asserted bound on $\mathfrak{a}$ follows from the above bound for $\mathfrak{c s}$ and $Q_{F}$, with the closed form $w_{f}$ in the definition of $Q_{F}$ replaced by $r^{-1} \varpi_{r}$.

5.2. The behavior of $\alpha, \beta$, and the curvature: Part 1 . This subsection derives various a priori bounds on the components of an instanton solution to (4.4). The desired bound on the spinor are summarized in the following lemma. This lemma states the analog here of Lemma 3.1 in [T4].

Lemma 5.2. There exists $\kappa \geq 1$ with the following significance: Suppose that $r \geq \kappa$ and that $(A, \psi=(\alpha, \beta))$ is an instanton solution to (4.4). Then

(1) $|\alpha| \leq 1+\kappa r^{-1}$.

(2) $|\beta|^{2} \leq \kappa r^{-1}\left(1-|\alpha|^{2}\right)+\kappa^{2} r^{-2}$.

The proof of this lemma uses the Bochner-Weitzenböck formula for the Dirac operator on $\mathbb{R} \times M$. The version that follows holds for any map $s \mapsto$ $\left.(A, \psi)\right|_{s}$ from $\mathbb{R}$ to $\operatorname{Conn}(E) \times C^{\infty}(M ; \mathbb{S})$. The relevant Dirac operator is

$$
\mathcal{D}_{A}=\frac{\partial}{\partial s}+D_{A}
$$


and the Bochner-Weitzenböck formula reads

$$
\mathcal{D}_{A}^{\dagger} \mathcal{D}_{A} \psi=\nabla_{\mathbb{A}}^{\dagger} \nabla_{\mathbb{A}} \psi-\mathrm{cl}\left(\frac{\partial}{\partial s} A+B_{A}\right) \psi+\frac{1}{4} R_{g} \psi,
$$

where the notation used on the right-hand side is as follows: First, $\psi$ is viewed as a section over $\mathbb{R} \times M$ of the pull-back of the spinor bundle $\mathbb{S}$. Second, $\nabla_{\mathbb{A}}$ here denotes the covariant derivative on this pull-back bundle that is defined by viewing $A$ and the canonical connection on $K^{-1}$ as connections over $\mathbb{R} \times M$ for the respective pull-backs of $E$ and $K^{-1}$. Finally, $R_{g}$ denotes the scalar curvature of the metric $g$ on $M$. The left-hand side of the preceding equation is zero when $(A, \psi)$ is an instanton.

Proof of Lemma 5.2. View $\mathbb{R} \times M$ as a four-dimensional Riemannian manifold so as to view the instanton equations as the four-dimensional SeibergWitten equations on $X=\mathbb{R} \times M$. Then, copy the arguments in Sections $2 \mathrm{a}-\mathrm{c}$ of the article $\mathrm{SW} \Rightarrow \mathrm{Gr}$ from [T6]. The latter use (5.5) with zero on the left-hand side and with $\frac{\partial}{\partial s} A+B_{A}$ given by (4.4) to derive differential equalities for $w=\left(1-|\alpha|^{2}\right)$ and $|\beta|^{2}$. In particular, these are of the following sort:

$$
\begin{aligned}
& \frac{1}{2} d^{\dagger} d w+r|\alpha|^{2} w-\left|\nabla_{A} \alpha\right|^{2}+\mathfrak{e}_{w}=0, \\
& \frac{1}{2} d^{\dagger} d|\beta|^{2}+r|\alpha|^{2}|\beta|^{2}+r\left(1+|\beta|^{2}\right)|\beta|^{2}+\left|\nabla_{A} \beta\right|^{2}+\mathfrak{e}_{\beta}=0,
\end{aligned}
$$

where

$$
\begin{aligned}
& \left|\mathfrak{e}_{w}\right| \leq c_{0}\left(|\alpha|^{2}+\left|\nabla_{A} \beta\right|^{2}+|\beta|^{2}\right) \quad \text { and } \\
& \left|\mathfrak{e}_{\beta}\right| \leq c_{0}\left(|\beta|^{2}+|\beta||\alpha|+|\beta|\left|\nabla_{A} \alpha\right|\right) .
\end{aligned}
$$

Here, $\nabla_{A}$ denotes the covariant derivative on sections over $\mathbb{R} \times M$ of the pull-backs of $E$ and $E \otimes K^{-1}$; it is defined by viewing $A$ and the canonical connection on $K^{-1}$ as connections on the respective pull-backs. As in Sections $2 \mathrm{a}-\mathrm{c}$ of the article $\mathrm{SW} \Rightarrow \mathrm{Gr}$ from [T6], the maximum principle is used with these inequalities to derive the bounds asserted by the lemma. The use of the maximum principle in this non-compact setting requires Lemma 3.5 to guarantee that the bounds given by the lemma hold as $s \rightarrow \pm \infty$.

The next lemma is the analog here of Lemma 3.2 in [T4], as it states bounds on $A$ and $B_{\mathbb{A}}$ for an instanton $\mathfrak{d}=(A, \psi)$. This lemma refers to

$$
\begin{aligned}
\mathcal{K} & =r \sup _{s \in \mathbb{R}} \underline{\mathrm{M}}(s), \quad \text { where } \\
\underline{\mathrm{M}}(s) & =\left.\int_{[s, s+1]}|1-| \alpha\right|^{2} \mid .
\end{aligned}
$$

Lemma 5.3. Fix a positive real number $L$. Then there exists a constant $\kappa>1$ depending onL, with the following significance: For any $r \geq \kappa$ and 
any solution $\mathfrak{d}=(A, \psi)$ to the $r$ 's version of instanton equation (4.4) with

$$
\Delta Q_{F}(\mathfrak{d}) \leq 2 \pi L
$$

one has

$$
\left|\frac{\partial}{\partial s} A-B_{A}\right| \leq r\left(1-|\alpha|^{2}\right)+\kappa\left(r^{3 / 4}+(\mathcal{K} r)^{1 / 2}\right) .
$$

This lemma is used in conjunction with the next lemma, which gives, among other things, a preliminary bound on $\mathcal{K}$.

Lemma 5.4. Fix a positive real number $L$. Then there exists a constant $\kappa>1$ depending on $L$, with the following significance: For any $r \geq \kappa$ and any solution $\mathfrak{d}=(A, \psi)$ to the $r$ 's version of instanton equation (4.4) satisfying (5.8), one has

$$
\begin{aligned}
\mathcal{K} & \leq \kappa r^{1 / 2}, \\
\int_{[s, s+1] \times M}\left(\left|B_{A}\right|^{2}+r\left|\nabla_{A} \psi\right|^{2}\right) & \leq \kappa r^{3 / 2} \quad \forall s \in \mathbb{R} .
\end{aligned}
$$

The proof of Lemma 5.4 in turn requires an a priori $L^{2}$-bound on $\mathbb{R} \times M$ for $\frac{\partial}{\partial s} A, \frac{\partial}{\partial s} \psi, D_{A} \psi$ and

$$
\mathfrak{B}_{A}:=B_{A}-r\left(\psi^{\dagger} \tau^{k} \psi-\mathrm{ia}\right)+\frac{1}{2} B_{0},
$$

where $B_{0}$ is as in (3.16), as follows:

Lemma 5.5. Fix a positive real number $L$. Then there exists a constant $\kappa>1$ depending on $L$, with the following significance: For any $r \geq \kappa$ and any solution $\mathfrak{d}=(A, \psi)$ to the $r$ 's version of instanton equation (4.4) satisfying (5.8), one has

$$
\int_{\mathbb{R} \times M}\left(\left|\frac{\partial}{\partial s} A\right|^{2}+\left|\mathfrak{B}_{A}\right|^{2}+2 r\left|\frac{\partial}{\partial s} \psi\right|^{2}+2 r\left|D_{A} \psi\right|^{2}\right) \leq \kappa r .
$$

Proof of Lemma 5.5. Fix $s>s^{\prime} \in \mathbb{R}$. The fact that (4.4) are the gradient flow equations for $\mathfrak{a}$ imply that

$$
\begin{aligned}
\int_{\left[s, s^{\prime}\right] \times M}\left(\left|\frac{\partial}{\partial s} A\right|^{2}+\left|\mathfrak{B}_{A}\right|^{2}+2 r\left|\frac{\partial}{\partial s} \psi\right|^{2}+2 r\left|D_{A} \psi\right|^{2}\right) & =-\Delta \mathfrak{a}\left(\left.\mathfrak{d}\right|_{\left[s, s^{\prime}\right]}\right), \\
\int_{\mathbb{R} \times M}\left(\left|\frac{\partial}{\partial s} A\right|^{2}+\left|\mathfrak{B}_{A}\right|^{2}+2 r\left|\frac{\partial}{\partial s} \psi\right|^{2}+2 r\left|D_{A} \psi\right|^{2}\right) & =-\Delta \mathfrak{a}(\mathfrak{d}) .
\end{aligned}
$$

Combining Lemma 5.1 and (5.4), we see that

$$
-\Delta \mathfrak{a}(\mathfrak{d}) \leq r\left(c_{0}+L\right) \quad \text { for some constant } c_{0} \text { depending only on } \mathfrak{c}_{-}, \mathfrak{c}_{+} .
$$

The assertion of the lemma follows by substituting this into the previous equality. 
Proof of Lemma 5.4. Use the first equation in (4.4) with the first two equations of Lemma 5.2 to see that

$$
\begin{aligned}
& \mathrm{i} \int_{\{s\} \times M} d t \wedge * \frac{\partial}{\partial s} A+\mathrm{i} \int_{\{s\} \times M} d t \wedge *\left(B_{A}+\frac{1}{2} B_{A_{K}}\right) \\
& \quad=\left.r \int_{\{s\} \times M}|1-| \alpha\right|^{2} \mid+\mathfrak{e},
\end{aligned}
$$

where $|\mathfrak{e}| \leq\left.\frac{r}{100} \int_{M}|1-| \alpha\right|^{2} \mid+c_{0}$. The second integral on the left-hand side of (5.11) is $\pi$ times the pairing between $c_{1}(\mathfrak{s})$ and the class represented by $F$ in $H_{2}(M ; \mathbb{R})$. This understood, integrate both sides of $(5.11)$ over $[s, s+1]$ and appeal to Lemma 5.5 to bound the $L^{1}$-norm of $\frac{\partial}{\partial s} A$ supplies the bound for $\mathcal{K}$ asserted by Lemma 5.4 .

The integral bound on $\left|B_{A}\right|^{2}$ asserted by Lemma 5.4 follows from the bound on $\mathcal{K}$, Lemma 5.2, the definition of $\mathfrak{B}_{A}$ and Lemma 5.5's bound on $\int_{M}\left|\mathfrak{B}_{A}\right|^{2}$. The integral bound for $r\left|\nabla_{A} \psi\right|^{2}$ asserted by Lemma 5.4 follows using the bound for $\mathcal{K}$, Lemma 5.2, Bochner-Weitzenböck formula for the square the Dirac operator on $M$, and the bound on $\int_{M}\left|D_{A} \psi\right|^{2}$ provided in Lemma 5.5.

Proof of Lemma 5.3. With (5.6), the arguments used to prove Lemma 2.5 in the article SW $\Rightarrow$ Gr in [T6] can be borrowed in a verbatim fashion to derive $(A, \psi)$ and $r$ independent constants $z_{1}, z_{2}, z_{3}$ and $z_{4}$, such that the following is true: Introduce the functions

$$
\begin{aligned}
q_{0} & =r\left(1+r^{-1} z_{1}\right)\left(1-|\alpha|^{2}\right)-z_{2} r|\beta|^{2}+z_{3} \\
s & =\left|\frac{\partial}{\partial s} A-B_{A}\right|, \\
q & =\max \left(s-q_{0}, 0\right) .
\end{aligned}
$$

The function $q$ obeys

$$
d^{\dagger} d q+2 r|\alpha|^{2} q \leq z_{4}\left(s+r\left(1-|\alpha|^{2}\right)\right) .
$$

As noted in the proof of Lemma 3.2 from [T4], the preceding equation for $q$ implies that

$$
d^{\dagger} d q-z_{4} q \leq c_{0} q_{0}+c_{0}
$$

Granted this, mimic what is done in the proof of Lemma 3.2 in [T4] with the Dirichlet Green's function for the operator $d^{\dagger} d-z_{4}$ on small radius balls in $\mathbb{R} \times M$ to conclude the following: If $\rho \in\left(0, c_{0}^{-1}\right)$, then

$$
\begin{aligned}
q(x) \leq & c_{0} \rho^{-4}\left(\int_{\operatorname{dist}(x, \cdot) \leq \rho}\left(\left|\frac{\partial}{\partial s} A\right|^{2}+\left|B_{A}\right|^{2}\right)\right)^{1 / 2} \\
& +c_{0} r \int_{\operatorname{dist}(x, \cdot) \leq \rho} \frac{1-|\alpha|^{2}}{\operatorname{dist}(x, \cdot)^{2}}+c_{0} .
\end{aligned}
$$


This uses the fact that the Green's function for $d^{\dagger} d-z_{4}$ with pole at $x$ obeys

$$
0 \leq G(\cdot, x) \leq c_{0} \operatorname{dist}(\cdot, x)^{-2} \quad \text { and } \quad|d G(\cdot, x)| \leq c_{0} \operatorname{dist}(\cdot, x)^{-3} .
$$

Use the integral bounds for $B_{A}$ in Lemma 5.4 and those for $\frac{\partial A}{\partial s}$ from Lemma 5.5 to bound the first term on the right side of (5.14) by $c_{0} r^{3 / 4}$. Fix $c \in(0, \rho)$ so as to consider the contribution to the right most integral in (5.14) from the part of the integration domain where $\operatorname{dist}(x, \cdot)>c$. This contribution is bounded by $c_{0} \mathcal{K} c^{-2}$. Meanwhile, the contribution from where $\operatorname{dist}(x, \cdot) \leq c$ is bounded by $c_{0} r c^{2}$. This understood, take $c=(\mathcal{K} r)^{-1 / 4}$ to bound the right most integral in (5.14) by $c_{0}(\mathcal{K} r)^{1 / 2}$. Thus,

$$
q(x) \leq c_{0}\left(r^{3 / 4}+(\mathcal{K} r)^{1 / 2}\right) .
$$

5.3. The behavior of $\alpha, \beta$, and the curvature: Part 2. The first lemma below concerns the size of the covariant derivatives of $\alpha$ and $\beta$. It is the analog of Lemma 3.6 in [T4]. This lemma and the subsequent discussions use $\nabla_{A}$ to denote the covariant derivative of a section of a bundle over $\mathbb{R} \times M$ as defined by viewing $A$ as a connection on the pull-back of $E$ over $\mathbb{R} \times M$. In particular, $\nabla_{A}$ has a component that differentiates along the $\mathbb{R}$ factor of $\mathbb{R} \times M$.

Lemma 5.6. Fix a positive real number $L$. Then there exists a constant $\kappa>1$ depending on $L$, with the following significance: For any $r \geq \kappa$ and any solution $\mathfrak{d}=(A, \psi)$ to the $r$ 's version of instanton equation (4.4) satisfying (5.8), one has

(1) $\left|\nabla_{A} \alpha\right|^{2} \leq \kappa r$.

(2) $\left|\nabla_{A} \beta\right|^{2} \leq \kappa$.

In addition, for each integer $q \geq 1$, there exists a constant $\kappa_{q}$ that depends only on $L$ and is such that when $r \geq \kappa$, then

(3) $\left|\nabla_{A}^{q} \alpha\right|+r^{1 / 2}\left|\nabla_{A}^{q} \beta\right| \leq \kappa_{q} r^{q / 2}$.

Proof of Lemma 5.6. These claims are local in nature and are proved by rescaling the Seiberg-Witten equation as written in Gaussian normal coordinates about any given point. Thus, the coordinate functions $\left\{x^{v}\right\}_{v=\{1,2,3,4\}}$ for the Gaussian coordinate chart are written as $x^{v}=r^{-1 / 2} y^{v}$. Uniform bounds on the curvature in the rescaled coordinates follow from Lemma 5.3. With the curvature bounded in the rescaled coordinate, uniform bounds on the covariant derivatives of rescaled sections can be obtained using standard elliptic regularity techniques. Undoing the rescaling gives the asserted bounds.

The next lemma refines Lemma 5.3's bound on the curvature. It is the analog here of Lemma 3.7 in [T4]. 
Lemma 5.7. Fix a positive real number $L$. Then there exists a constant $\kappa>1$ depending onL, with the following significance: For any $r \geq \kappa$ and any solution $\mathfrak{d}=(A, \psi)$ to the $r$ 's version of instanton equation (4.4) satisfying (5.8), one has

$$
\left|\frac{\partial}{\partial s} A-B_{A}\right| \leq r\left(1+\kappa \mathcal{K}^{1 / 2} r^{-1 / 2}\right)\left(1-|\alpha|^{2}\right)+\kappa .
$$

Proof. Reintroduce the function $q$ from the proof of Lemma 5.3. It follows from (5.13) and from Lemma 5.3 that a constant $z_{5} \geq 1$ can chosen independently of $(A, \psi)$ and $r$ so that the function

$$
q_{1}=\max \left(q-z_{5}, 0\right)
$$

obeys

$$
d^{\dagger} d q_{1}+2 r|\alpha|^{2} q_{1} \leq c_{0} r\left(1-|\alpha|^{2}\right)
$$

in the weak sense.

Given (5.6), what is done in the proof of Lemma 2.7 in the article $\mathrm{SW} \Rightarrow$ Gr from [T6] when repeated here finds constants, $\delta_{1}>0$ and $\delta_{2}>0$, both independent of $r$ and of $(A, \psi)$, and such that the function

$$
\mathrm{v}_{1}=1-|\alpha|^{2}+r^{-1} \delta_{1}-\delta_{2}|\beta|^{2}
$$

has the following properties when $r \geq c_{0}$ :

$$
\begin{aligned}
& \mathrm{v}_{1} \geq r^{-1} \delta ; \\
& \mathrm{v}_{1} \geq\left.|1-| \alpha\right|^{2} \mid \\
& d^{\dagger} d \mathrm{v}_{1}+2 r|\alpha|^{2} \mathrm{v}_{1} \geq 0 .
\end{aligned}
$$

Set

$$
\varepsilon=r^{-1 / 2} \quad \text { and } \quad \mathrm{v}_{2}=\mathrm{v}_{1}^{1-\varepsilon} .
$$

It follows from the second inequality in (5.17) that the function $\mathrm{v}_{2}$ also obeys

$$
\mathrm{v}_{2} \geq\left.|1-| \alpha\right|^{2} \mid \text {. }
$$

This and the third inequality in (5.17) imply that

$$
d^{\dagger} d \mathrm{v}_{2}+2 r|\alpha|^{2} \mathrm{v}_{2} \geq 2 r^{1 / 2}|\alpha|^{2}\left(1-|\alpha|^{2}\right) .
$$

Granted the latter equation, it then follows from (5.16) that there is a constant $z_{6} \geq 0$ with the following properties: First, $z_{6}$ is independent of both $r$ and $(A, \psi)$. To state the second, introduce the function

$$
v=q_{1}-z_{6}\left(r^{1 / 2}+\left\|q_{1}\right\|_{\infty}\right) \mathrm{v}_{2} .
$$

Then this function obeys:

(1) $v<0$ at points where $|\alpha|^{2}=\frac{1}{2}$;

(2) $d^{\dagger} d v+2 r|\alpha|^{2} v \leq 0$ at points where $|\alpha|^{2} \geq \frac{1}{2}$. 
This last inequality with the maximum principle finds

$$
v \leq 0 \quad \text { where }|\alpha|^{2} \geq \frac{1}{2} .
$$

This implies the assertion of Lemma 5.7 at points where $|\alpha|^{2} \geq \frac{1}{2}$. The assertion at points where $|\alpha|^{2} \leq \frac{1}{2}$ follows directly from Lemma 5.3.

5.4. Bounds for the integral of $\boldsymbol{r}\left(1-|\boldsymbol{\alpha}|^{2}\right)$. Lemma 5.4 bounds what (5.7) calls $\mathcal{K}$ by $c_{0} r^{1 / 2}$. The next lemma asserts that $\mathcal{K}$ has an $r$-independent bound.

Lemma 5.8. Fix a positive real number $L$. Then there exists a constant $\kappa>1$ depending on $L$, with the following significance: For any $r \geq \kappa$ and any solution $\mathfrak{d}=(A, \psi)$ to the $r$ 's version of instanton equation (4.4) satisfying (5.8), one has

$$
\mathcal{K} \leq \kappa
$$

Proof. As noted in Section 3.3, the fact that $J$ is tamed by the symplectic form $d s \wedge d t+w_{f}$ requires that the one-form $a$ that appears in (3.11) have norm less than 2. This understood, introduce

$$
\delta=2-\sup _{M}|a| \text {. }
$$

Recall from (5.7) that $\mathcal{K}$ is defined as the supremum of $r \underline{\mathrm{M}}(s)$. Without loss of generality, suppose that this supremum is attained at a point $s \in \mathbb{R}$. Otherwise, if $\underline{\mathrm{M}}(s) \neq r \mathcal{K} \forall s$, then

$$
\mathcal{K}=\lim _{s \rightarrow \infty} r \underline{\mathrm{M}}(s) \quad \text { or } \quad \lim _{s \rightarrow-\infty} r \underline{\mathrm{M}}(s) .
$$

Since $(A(s), \psi(s))$ converges to a solutions of (3.13) when $s \rightarrow \infty$ or $-\infty$, the assertion of the lemma then follows from Lemma 3.6. by

Recall the function $\chi$ from Section 1.4, and define the function $\chi_{s}$ on $\mathbb{R}$

$$
\chi_{s}(\cdot)=\chi\left(\frac{1}{4}|s-(\cdot)|\right) .
$$

In particular, this function has compact support in the interval $[s-2, s+2]$, but it is equal to 1 on $[s, s+1]$. As will be explained momentarily, for any $\varepsilon \in(0,1)$, there exists a constant $c_{\varepsilon} \geq 1$ that is independent of $r, \delta$, and $(A, \psi)$, so that the following holds:

$$
\delta \mathcal{K} \leq 2 \int_{\mathbb{R} \times M} \chi_{s}\left(r|\alpha|^{2}\left(1-|\alpha|^{2}\right)-\left|\nabla_{A} \alpha\right|^{2}\right)+c_{\varepsilon}+c_{0} \varepsilon \mathcal{K} \quad \text { when } r \geq c_{\varepsilon}
$$

Note that the integral in (5.19) is bounded by $c_{0}$ via the following argument: Multiply both sides of the first equation in (5.6) by the function $\chi_{s}$ and integrate the resulting equation over $\mathbb{R} \times M$. Then the claimed bound follows from integration by parts, Lemmas 5.2 and 5.6. 
This understood, then any $\varepsilon<c_{0}^{-1} \delta$ version of (5.19) supplies an $r$-independent bound on $\mathcal{K}$.

The derivation of (5.19) is given next in five steps.

Step 1. In this step $\nabla_{\mathbb{A}}$ or $\nabla_{A}$ denotes the covariant derivative on bundles over $\mathbb{R} \times M$ as in (5.5), and use $\nabla_{\AA}^{M}$ or $\nabla_{A}^{M}$ to denote the corresponding covariant derivative along the $M$-direction. The identity (5.5) has an analog for any given pair $(A, \psi) \in \operatorname{Conn}(E) \times C^{\infty}(M ; \mathbb{S})$, with $\mathcal{D}_{A}$ replaced by $D_{A}$, with $\nabla_{\mathbb{A}}$ replaced by $\nabla_{\mathbb{A}}^{M}$, and with $\frac{\partial}{\partial s} A$ set to zero there. Integrate both sides of this identity over $M$; then integrate by parts on both sides to find

$$
\begin{aligned}
& \int_{M}\left(\left|B_{A}-r\left(\psi^{\dagger} \tau \psi-\mathrm{ia}\right)\right|^{2}+2 r\left|D_{A} \psi\right|^{2}\right) \\
& \quad=\int_{M}\left(\left|B_{A}\right|^{2}+r^{2}\left|\psi^{\dagger} \tau \psi-\mathrm{ia}\right|^{2}-2 \mathrm{i} r \mathrm{a} \wedge * B_{A}+2 r\left(\left|\nabla_{A}^{M} \psi\right|^{2}+\frac{R_{g}}{4}|\psi|^{2}\right)\right) .
\end{aligned}
$$

Now take the pair $(A, \psi)$ above to be the restriction to a slice $\left\{s^{\prime}\right\} \times M$ of the instanton solution to (4.4), and regard either side of the identity as a function on $s^{\prime} \in \mathbb{R}$. Multiply this function by $\chi_{s}\left(s^{\prime}\right)$ and integrate over $s^{\prime} \in \mathbb{R}$ to see the equality of the left-hand sides of Equations (5.21) and (5.22) below.

Noting that $B_{A}-r\left(\psi^{\dagger} \tau \psi-\right.$ ia $)=\mathfrak{B}_{A}-\frac{1}{2} B_{0}($ see $(5.9))$, use Lemma 5.5 to see that

$$
\begin{gathered}
\int_{\mathbb{R} \times M} \chi_{s}\left(\left|B_{A}-r\left(\psi^{\dagger} \tau \psi-\mathrm{ia}\right)\right|^{2}+2 r\left|D_{A} \psi\right|^{2}\right) \\
\leq \int_{\mathbb{R} \times M} \chi_{s}\left(\left|\mathfrak{B}_{A}\right|^{2}+2 r\left|D_{A} \psi\right|^{2}\right)+c_{0} r^{1 / 2} .
\end{gathered}
$$

Write a as $d t-a$ as in (3.11), and recall that $a$ is in the space of one-forms that annihilate $\partial_{t}$. Denote the latter space by $\Omega^{\perp}$. Use Lemmas 5.2 and 5.5 to find

$$
\begin{gathered}
\int_{\mathbb{R} \times M} \chi_{s}\left(\left|B_{A}\right|^{2}+r^{2}\left|\psi^{\dagger} \tau \psi-\mathrm{ia}\right|^{2}-2 \mathrm{i} r \mathrm{a} \wedge *_{A}+2 r\left(\left|\nabla_{A}^{M} \psi\right|^{2}+\frac{R_{g}}{4}|\psi|^{2}\right)\right) \\
>\int_{\mathbb{R} \times M} \chi_{s}\left(\left|B_{A}\right|^{2}+\left.\left.r^{2}|1-| \alpha\right|^{2}\right|^{2}+2 r\left|\nabla_{A} \alpha\right|^{2}-2 r|a|\left|B^{\perp}\right|\right)-c_{0} r,
\end{gathered}
$$

where $B^{\perp}$ denotes the orthogonal projection of $B_{A}$ to $\Omega^{\perp}$. Note that the derivation of (5.22) uses the fact that

$$
\int_{M} d t \wedge i * B_{A}=4 \pi^{2} d_{\Gamma}
$$


Step 2. Write $\frac{\partial}{\partial s} A$ and $B_{A}$ as

$$
\begin{aligned}
\frac{\partial}{\partial s} A & =-i(1-v)\left(r\left(1-|\alpha|^{2}\right)+\mathfrak{z}\right) \mathrm{a}+\mathfrak{x}+\mathfrak{X} \quad \text { and } \\
B_{A} & =-i v\left(r\left(1-|\alpha|^{2}\right)+\mathfrak{z}\right) \mathbf{a}+\mathfrak{x}-\mathfrak{X},
\end{aligned}
$$

where:

- $v$ is a function on $\mathbb{R} \times M$;

- $\mathfrak{z}=r|\beta|^{2}-\frac{\mathrm{i}}{2} *\left(\mathrm{a} \wedge * B_{A_{K}}\right)$;

- both $i \mathfrak{X}$ and $i \mathfrak{x}$ are in $\Omega^{\perp}$.

It is a consequence of Lemma 5.2 that $|\mathfrak{z}| \leq c_{0}$. Meanwhile, the form $\mathfrak{x}$ is constrained by the first equation in (4.4). In particular, the latter and Lemma 5.2 require that

$$
|\mathfrak{x}| \leq c_{0}\left(\left.\left.r^{1 / 2}|1-| \alpha\right|^{2}\right|^{1 / 2}+1\right) .
$$

Let $w$ denote $\left(1-|\alpha|^{2}\right)$. The norm of $\mathfrak{X}$ is constrained using Lemmas 5.4 and 5.7 to obey

$$
4|\mathfrak{X}|^{2}+(1-2 v)^{2} r^{2} w^{2} \leq r^{2}\left(1+c_{0} \mathcal{K}^{1 / 2} r^{-1 / 2}\right)^{2} w^{2}+c_{0}(r|w|+1) .
$$

Given that Lemma 5.4 finds $\mathcal{K} \leq c_{0} r^{1 / 2}$, this last inequality requires

$$
\begin{aligned}
|\mathfrak{X}|^{2} \leq & r^{2} v(1-v) w^{2}+c_{0}\left(r^{7 / 4} w^{2}+r|w|+1\right), \\
& -c_{0} r^{-1 / 4} \leq v \leq 1+c_{0} r^{-1 / 4}
\end{aligned}
$$

Step 3. For the rest of the argument we shall frequently use the fact that $|w| \leq 1$ by Lemma 5.2 to bound $w^{2}$ by $|w|$ without further mention.

Let $\varepsilon \in(0,1)$. It follows from (5.20)-(5.22), (5.24), (5.26), and Lemma 5.5 that

$$
\begin{aligned}
& \int_{\mathbb{R} \times M} \chi_{s}\left(2 \varepsilon^{-1}\left|\frac{\partial}{\partial s} A\right|^{2}+\left|B_{A}\right|^{2}+r^{2} w^{2}+2 r\left|\nabla_{A} \alpha\right|^{2}-r^{2}|a||w|\right) \\
& \leq c_{0} r\left(\varepsilon^{-1}+\varepsilon \mathcal{K}\right)
\end{aligned}
$$

when $r \geq c_{\varepsilon}$ with $c_{\varepsilon} \geq 1$ a constant that depends solely on $\varepsilon$.

Step 4. Use (5.23) to see that

$$
\int_{\mathbb{R} \times M} \chi_{s}\left|\frac{\partial}{\partial s} A\right|^{2}=\int_{\mathbb{R} \times M} \chi_{s}\left((v-1)^{2} r^{2} w^{2}+|\mathfrak{X}|^{2}\right)+\mathfrak{e}_{A},
$$

where $\left|\mathfrak{e}_{A}\right| \leq c_{0} r\left(\varepsilon^{-1}+\varepsilon \mathcal{K}\right)$. Likewise,

$$
\int_{\mathbb{R} \times M} \chi_{s}\left|B_{A}\right|^{2}=\int_{M} \chi_{s}\left(v^{2} r^{2} w^{2}+|\mathfrak{X}|^{2}\right)+\mathfrak{e}_{B},
$$


where $\left|\mathfrak{e}_{B}\right| \leq c_{0} r\left(\varepsilon^{-1}+\varepsilon \mathcal{K}\right)$. Thus,

$$
\begin{aligned}
& \int_{\mathbb{R} \times M} \chi_{s}\left(2 \varepsilon^{-1}\left|\frac{\partial}{\partial s} A\right|^{2}+\left|B_{A}\right|^{2}-r^{2} w^{2}\right) \\
& \left.\quad \geq \int_{\mathbb{R} \times M} \chi_{s}\left(\left(1+2 \varepsilon^{-1}\right) v^{2}-4 \varepsilon^{-1} v+2 \varepsilon^{-1}-1\right) r^{2} w^{2}\right)+2 \varepsilon^{-1} \mathfrak{e}_{A}+\mathfrak{e}_{B} \\
& \quad \geq-c_{0} r\left(\varepsilon^{-2}+\varepsilon \mathcal{K}\right)
\end{aligned}
$$

for all sufficiently large $r$, since

$$
\left(1+2 \varepsilon^{-1}\right) v^{2}-4 \varepsilon^{-1} v+2 \varepsilon^{-1}-1 \leq 4\left(1-\varepsilon^{-1}\right)\left(2 \varepsilon^{-1}+1\right)^{-1}<0 .
$$

Combine this inequality with (5.27) to get

$$
\begin{aligned}
& \int_{\mathbb{R} \times M} \chi_{s}\left(2 r^{2} w^{2}+2 r\left|\nabla_{A} \alpha\right|^{2}-r^{2}|a||w|\right) \\
& \quad \leq c_{0} r\left(\varepsilon^{-2}+\varepsilon \mathcal{K}\right)
\end{aligned}
$$

when $r \geq c_{\varepsilon}$ for different values of $c_{0}$ and $c_{\varepsilon}$.

Step 5. Recall the notation $\sup _{M}|a|=2-\delta$; it follows immediately from (5.31) that

$$
\delta \int_{\mathbb{R} \times M} \chi_{s} r^{2}|w|+2 r \int_{\mathbb{R} \times M} \chi_{s}\left(r w^{2}+\left|\nabla_{A} \alpha\right|^{2}-r|w|\right) \leq c_{0} r\left(\varepsilon^{-2}+\varepsilon \mathcal{K}\right) .
$$

Use the first inequality in Lemma 5.2 to see that this inequality also holds with $|w|$ replaced by $w+c_{0} r^{-1}$ in the right most integrand on the left side of this inequality. Make this replacement. The resulting inequality directly implies (5.19).

Remark. By imposing additional conditions on the choice of the almost complex structure $J$, the proofs of Lemmas 5.3-5.8 may be simplified.

When the degree of $\Gamma$ is larger than $g-1$ or less than 2 , one may set $a=0$ (i.e., $J$ is admissible) yet still have the transversality and compactness results required for the definition of the periodic Floer homology, see the arguments in $[\mathbf{H 1}]$. In this case, Lemma 5.8 follows directly from taking the squares of both sides of the Seiberg-Witten equations (4.4) and the monotonicity of the action functional, without making use of Lemmas 5.3, 5.4, and the somewhat involved argument in the above proof.

In general, $a$ may be taken to be arbitrarily small for the purpose of the aforementioned transversality and compactness results. Assuming this, the final two steps of the preceding argument may be omitted. 
5.5. Monotonicity for the integral of $r\left(1-|\alpha|^{2}\right)$. The lemma that follows plays a key role in the proof of the surjectivity of $\Psi^{r}$. Denote by $\mathrm{M}$ the function from $(\mathbb{R} \times M) \times(0, \infty) \rightarrow(0, \infty)$ that assigns to a given pair $(\rho, x)$ the number

$$
\mathrm{M}(x, \rho)=\left.r \int_{\operatorname{dist}(x, \cdot) \leq \rho}|1-| \alpha\right|^{2} \mid .
$$

The upcoming lemma gives upper and lower bounds for this function. It is the analog of Lemma 3.10 in [T4] and Proposition 3.1 in the article $\mathrm{SW} \Rightarrow$ Gr from [T6].

Lemma 5.9. Fix a positive real numbers $L$. Then there exists a constant $\kappa>1$ depending on $L$, with the following significance: For any $r \geq \kappa$ and any solution $\mathfrak{d}=(A, \psi)$ to the $r$ 's version of instanton equation (4.4) satisfying (5.8), one has

(1) If $\rho_{1}>\rho_{0} \in\left(r^{-1 / 2}, \kappa^{-1}\right)$, then for any $x \in \mathbb{R} \times M$,

$$
\mathrm{M}\left(x, \rho_{1}\right) \geq \kappa^{-1} \rho_{1}^{2} / \rho_{0}^{2} \mathrm{M}\left(x, \rho_{0}\right) .
$$

(2) Suppose that $|\alpha(x)| \leq \frac{1}{2}$ at $x$. If $\rho \in\left(r^{-1 / 2}, \kappa^{-1}\right)$, then

$$
\kappa^{-1} \rho^{2} \leq \mathrm{M}(x, \rho) \leq \kappa \rho^{2} .
$$

Proof. To start, let

$$
\omega=d s \wedge \mathrm{a}+w_{f}
$$

View $A$ as a connection on the pull-back of the bundle $E$ to $\mathbb{R} \times M$ and use $F_{A}$ to denote its curvature two-form. The latter is equal to $d s \wedge \frac{\partial}{\partial s} A+* B_{A}$ where it is understood that $*$ here refers to the Hodge star for the metric on $M$. Granted this notation, it then follows using (4.4) with Lemma 5.2 that

$$
\mathrm{M}(x, \rho)=\int_{\operatorname{dist}(x, \cdot) \leq \rho} \mathrm{i} \omega \wedge F_{A}+\mathfrak{e}_{1},
$$

where $\left|\mathfrak{e}_{1}\right| \leq c_{0} \rho^{4}$. Now fix Gaussian coordinates $\left(y_{1}, \ldots, y_{4}\right)$ centered at $x$, chosen so that

$$
\omega=d y_{1} \wedge d y_{2}+d y_{3} \wedge d y_{4}+\mathcal{O}(|y|) .
$$

Set $\omega_{0}=d y_{1} \wedge d y_{2}+d y_{3} \wedge d y_{4}$. It then follows from (5.34) with Lemmas 5.2 and 5.8 that

$$
\mathrm{M}(x, \rho)=\int_{\operatorname{dist}(x, \cdot) \leq \rho} \mathrm{i} \omega_{0} \wedge F_{A}+\mathfrak{e}_{2},
$$

where $\left|\mathfrak{e}_{2}\right| \leq c_{0}\left(\rho^{4}+\rho \mathrm{M}(x, \rho)\right)$. Next note that $\omega_{0}=d \theta_{0}$, where

$$
\theta_{0}=\frac{1}{2}\left(y_{1} d y_{2}-y_{2} d y_{1}+y_{3} d y_{4}-y_{4} d y_{3}\right) \text {. }
$$


Note in particular that $\left|\theta_{0}\right|=\frac{1}{2}|y|+\mathfrak{e}_{3}$ where $\left|\mathfrak{e}_{3}\right| \leq c_{0}|y|^{2}$. Write the integral in $(5.36)$ as

$$
\int_{\operatorname{dist}(x, \cdot) \leq \rho} \mathrm{i} \theta_{0} \wedge\left(F_{A}\right)^{T},
$$

where $\left(F_{A}\right)^{T}$ denotes the restriction of $F_{A}$ to the tangent space of the sphere of radius $\rho$ into $\mathbb{R} \times M$.

To continue, note that

$$
\left|\theta \wedge\left(F_{A}\right)^{T}\right| \leq\left.\frac{1}{2} \rho r\left(1+c_{0} r^{-1 / 2}\right)(1+\rho)|1-| \alpha\right|^{2} \mid+c_{0} \rho .
$$

Indeed, this follows from the bound

$$
\left|\theta_{0}\right| \leq \frac{1}{2}|y|+c_{0}|y|^{2}
$$

with the following two facts: First,

$$
\left|\frac{\partial}{\partial s} A+B_{A}\right| \leq r\left(1-|\alpha|^{2}\right)+c_{0}
$$

which is a consequence of (4.4) and Lemma 5.2. Second,

$$
\left|\frac{\partial}{\partial s} A-B_{A}\right| \leq r\left(1+c_{0} r^{-1 / 2}\right)\left(1-|\alpha|^{2}\right)+c_{0},
$$

which is a consequence of Lemmas 5.7 and 5.8.

Granted these bounds, it follows from (5.36) and (5.38) that the function $\rho \mapsto \mathrm{M}(x, \rho)$ obeys the differential inequality

$$
\mathrm{M}(x, \rho) \leq \frac{1}{2} \rho\left(1+c_{0} \rho+c_{0} r^{-1 / 2}\right) \frac{\partial \mathrm{M}}{\partial \rho}(x, \rho)+c_{0} \rho^{4} .
$$

Integration of (5.40) gives the what is asserted in item (1) of Lemma 5.9. The left-hand inequality of item (2) follows from item (1), since

$$
\mathrm{M}\left(x, r^{-1 / 2}\right) \geq c_{0}^{-1} r^{-1} \quad \text { if }|\alpha(x)| \leq \frac{1}{2} .
$$

Indeed such an upper bound follows directly from the first inequality in Lemma 5.6. The right-hand inequality of item (2) follows from item (1) and Lemma 5.8.

5.6. The behavior of $\alpha, \beta$ and the curvature: Part 3. The bounds given below refine the bounds given in Lemmas 5.6 and 5.7. The first lemma below states the analog here of what is asserted by Propositions 2.8 and 4.4 of the article $\mathrm{SW} \Rightarrow \mathrm{Gr}$ in [T6]. It is the analog of Lemma 3.8 in [T4].

Lemma 5.10. Fix a positive real number $L$. Then there exists a constant $\kappa>1$ depending on $L$, with the following significance: Fix an $r \geq \kappa$ and $a$ 
solution $\mathfrak{d}=(A, \psi)$ to the $r$ 's version of instanton equation (4.4) satisfying (5.8). Set $X_{*}$ to denote the subset in $\mathbb{R} \times M$ where $1-|\alpha| \geq \kappa^{-1}$. Then

(1) $\left|\nabla_{A} \alpha\right|^{2}+r\left|\nabla_{A} \beta\right|^{2} \leq \kappa r\left(1-|\alpha|^{2}\right)+\kappa^{2}$;

(2) $r\left(1-|\alpha|^{2}\right)+\left|\nabla_{A} \alpha\right|^{2}+r\left|\nabla_{A} \beta\right|^{2} \leq \kappa\left(r^{-1}+r \mathrm{e}^{-\sqrt{r} \operatorname{dist}\left(\cdot, X_{*}\right) / \kappa}\right)$;

(3) $|\beta|^{2} \leq \kappa\left(r^{-2}+r^{-1} \mathrm{e}^{-\sqrt{r} \operatorname{dist}\left(\cdot, X_{*}\right) / \kappa}\right)$.

As in Lemma 5.6, what is written as $\nabla_{A}$ refers to the covariant derivative over $\mathbb{R} \times M$ as defined by viewing $A$ as a connection on the pull-back of the bundle $E$ to $\mathbb{R} \times M$.

Proof of Lemma 5.10. The proof differs in one place from the proof of Lemma 3.8 in [T4]. Here is the argument in our case: Use Lemma 5.7 with the manipulations done in Step 2 from the proof of Proposition 4.4 in the article $\mathrm{SW} \Rightarrow \mathrm{Gr}$ in $[\mathbf{T 6}]$ to obtain an $r$ and $(A, \psi)$ independent constant $z_{*} \geq 1$ such that

$$
y=\left|\nabla_{A} \alpha\right|^{2}+r\left|\nabla_{A} \beta\right|^{2}-z_{*}
$$

obeys the differential inequality

$$
d^{\dagger} d y+2 r|\alpha|^{2} y \leq c_{0} r\left(1-|\alpha|^{2}\right) y+c_{0} y
$$

at points on $\mathbb{R} \times M$ where $1-|\alpha|^{2} \leq c_{0}^{-1}$. Meanwhile, use (5.6) with Lemma 5.1 to obtain an $(A, \psi)$ and $r$ independent constant $z_{* *}$ such that the function

$$
w=\left(1-|\alpha|^{2}\right)-z_{* *}|\beta|^{2}
$$

obeys

$$
-c_{0}+y \leq d^{\dagger} d w+2 r|\alpha|^{2} w \leq c_{0}(y+1) .
$$

Fix $c_{*} \geq 1$ and then use (5.41) and the left most inequality in (5.42) to see that

$$
u=\max \left(y-c_{*}\left(\|y\|_{\infty}+r+1\right) w-c_{0} c_{*}, 0\right)
$$

obeys the differential inequality

$$
d^{\dagger} d u+\frac{r}{64} u \leq 0
$$

on the domain $U \subset \mathbb{R} \times M$ where $1-|\alpha|^{2} \leq c_{0}^{-1}$. If $c_{*} \geq c_{0}$, then $u$ is negative on the boundary of $U$ and it has compact support, the latter being a consequence of Lemma 3.5. The maximum principle demands $u=0$ which proves item (1) of the lemma for points in $U$. Meanwhile, Lemma 3.5 and the fact that $w>c_{0}^{-1}$ on the complement of $U$ imply item (1) on the complement of $U$.

To obtain the assertion of item (2), use (5.41) with the left-hand inequality in (5.42) to see that

$$
u^{\prime}=\max \left(y+c_{0}^{-1} r w-c_{0}, 0\right)
$$


obeys

$$
d^{\dagger} d u^{\prime}+\frac{r}{64} u^{\prime} \leq 0 \quad \text { in } U
$$

Keeping this in mind, let $c_{M}>0$ denote a constant that is much less than the injectivity radius of $M$. Let $x \in X_{*}$ denote a point with $s(x) \in\left[s_{0}-R, s_{0}+R\right]$; and let $B \subset X_{*}$ denote the ball with center $x$ and radius equal to half of the minimum of $c_{M}$ and $\operatorname{dist}\left(x, X_{*}\right)$. Use $\rho$ to denote the radius of the ball $B$. Let $G(\cdot, x)$ denote the Green's function for the operator $d^{\dagger} d+\frac{r}{64}$ with pole at $x$. This operator obeys the bounds

$$
\begin{gathered}
0 \leq G(\cdot, x) \leq c_{0} \operatorname{dist}(\cdot, x)^{-2} \mathrm{e}^{-\sqrt{r} \operatorname{dist}(\cdot, x) / c_{0}} \quad \text { and } \\
|d G(\cdot, x)| \leq c_{0} \operatorname{dist}(\cdot, x)^{-3} \mathrm{e}^{-\sqrt{r} \operatorname{dist}(\cdot, x) / c_{0}} .
\end{gathered}
$$

Multiply both sides of the inequality

$$
d^{\dagger} d u^{\prime}+\frac{r}{64} u^{\prime} \leq 0
$$

by $\chi(\operatorname{dist}(\cdot, x) / \rho) G(\cdot, x)$ and integrate by over $B$. Given that $\left|u^{\prime}\right| \leq c_{0} r$, integration by parts finds

$$
\left.u^{\prime}\right|_{x} \leq c_{0} r \mathrm{e}^{-\sqrt{r} \rho / c_{0}} .
$$

This implies what is asserted by the second item of the lemma. The third item follows from the second using Lemma 5.2.

The next lemma refines the bounds given by Lemma 5.7 for the curvature. This lemma is the analog of Proposition 3.4 in the article $\mathrm{SW} \Rightarrow$ Gr from [T6], and it is the analog of Lemma 3.9 in [T4].

Lemma 5.11. Given $\mathcal{K} \geq 1$, there exists $\kappa \geq 1$ with the following significance: Suppose that $r \geq \kappa$, and that $(A, \psi=(\alpha, \beta))$ is an instanton solution to (4.4). Fix $s_{0} \in \mathbb{R}$ and $R \geq 1$; and suppose that

$$
\sup _{s \in\left[s_{0}-R-3, s_{0}+R+3\right]} \underline{\mathrm{M}}(s) \leq \mathcal{K} .
$$

Then

$$
\begin{aligned}
& \left|\frac{\partial A}{\partial s}+B_{A}\right| \leq r\left(1-|\alpha|^{2}\right)+\kappa, \\
& \left|\frac{\partial A}{\partial s}-B_{A}\right| \leq r\left(1-|\alpha|^{2}\right)+\kappa
\end{aligned}
$$

at all points in $\left[s_{0}-R, s_{0}+R\right] \times M$.

Proof. Copy the proof of Lemma 3.9 in [T4] using Lemmas 5.2, 5.7-5.9 to replace their [T4] analogs.

Arguments from [T4] refer to a connections on the pull-back of $E$ over $\mathbb{R} \times M$ that are denoted by $\hat{A}$. Each version of $\hat{A}$ differs from $A$ where $|\alpha|$ is near to 1 . Any given version is defined from a specified function $\wp:[0, \infty) \rightarrow$ 
$[0, \infty)$ which is a non-decreasing function that obeys $\wp(x)=x$ for $x$ near zero and $\wp(1)=1$. With $\wp$ in hand, set

$$
\hat{A}=A-\frac{1}{2} \wp\left(|\alpha|^{2}\right)|\alpha|^{-2}\left(\bar{\alpha} \nabla_{A} \alpha-\alpha \nabla_{A} \bar{\alpha}\right) .
$$

Here, and as previously, $\nabla_{A}$ denotes the covariant on $\mathbb{R} \times M$ as defined by $A$. The curvature, $F_{\hat{A}}$, of this connection is

$$
F_{\hat{A}}=(1-\wp) F_{A}-\wp^{\prime} \nabla_{A} \bar{\alpha} \wedge \nabla_{A} \alpha .
$$

If the assumptions of Lemma 5.10 hold, then

$$
\left|F_{\hat{A}}\right| \leq c_{0}\left(r^{-1}+r \mathrm{e}^{-\sqrt{r} \operatorname{dist}\left(\cdot, X_{*}\right) / \kappa}\right)
$$

on the whole of $\mathbb{R} \times M$.

5.7. Behavior near $\mathbb{R} \times \gamma$ when $\gamma$ is elliptic. The following is the analog here of Lemma 3.11 in [T4]:

Lemma 5.12. Fix a positive real number $L$. Then there exists a constant $\kappa>1$ depending on $L$, with the following significance: For any $r \geq \kappa$ and any solution $\mathfrak{d}=(A, \psi)$ to the $r$ 's version of instanton equation (4.4) satisfying (5.8), and for any elliptic periodic orbit $\gamma$ with tubular neighborhood map as described by (4.1), one has:

$$
|\beta| \leq \kappa r^{-1}, \quad\left|\nabla_{A} \beta\right| \leq \kappa r^{-1 / 2}
$$

at all points in $\mathbb{R} \times M$ with distance $\kappa^{-1}$ or less from $\mathbb{R} \times \gamma$.

Proof. Copy the proof of Lemma 3.11 in [T4].

\section{Generalizing to the non-monotone case}

In the non-monotone case of either the Periodic Floer homology or SeibergWitten-Floer cohomology, the definition of the Floer (co)homology in Section 1.1 or 1.2 no longer works, because the moduli space $\mathcal{M}_{1}\left(\Theta_{+}, \Theta_{-}\right)$or $\mathcal{M}_{1}\left(\mathfrak{c}_{-}, \mathfrak{c}_{+}\right)$used to define the differential fails to be compact. To make sense of the infinite sum in (1.9) or (1.16), the standard solution from the Floer theory literature is to work with Novikov coefficients. This is done in the Seiberg-Witten context in Chapter 30 in $[\mathbf{K M}]$. We shall follow the formulation of Kronheimer and Mrowka, with notation and terminology modified so as to better accommodate both versions of Floer (co)homologies under discussion in one common framework. By way of a parenthetical remark, the formulation used in [T5] for embedded contact homology and SeibergWitten-Floer cohomology with group ring coefficients can be modified so as to apply in the present context with Novikov coefficients. 
6.1. Local coefficients. We shall use the term "local system" in the following more general sense: In place of topological spaces, consider data sets of the following type.

Definition 6.1. A fundamental triple consists of:

- a category $\mathfrak{G}$;

- a groupoid $\underline{\mathfrak{G}}$ with the same objects as $\mathfrak{G}$, and such that for every pair $a, b \in \mathcal{O} \mathfrak{b}(\mathfrak{G})$, the set of morphisms $\mathcal{M o r}(\underline{\mathfrak{G}} ; a, b)$ in $\underline{\mathfrak{G}}$ from $a$ to $b$ has countably many elements;

- a functor $\pi_{\mathfrak{G}}$ from the former to the latter, which identifies the objects in both categories.

The model example. Take $\mathfrak{G}$ to be the category of points and paths in a topological space of countable $\pi_{1}$; let $\underline{\mathfrak{G}}$ be the fundamental groupoid of this space, and $\pi_{\mathfrak{G}}$ defined by taking the relative homotopy class.

Definition 6.2. Let $R$ be a commutative ring, and let $\operatorname{Mod}_{R}$ denote the category of $R$-modules. Given a fundamental triple $\pi_{\mathfrak{G}}: \mathfrak{G} \rightarrow \underline{\mathfrak{G}}$, we call a functor $\Lambda$ from $\underline{\mathfrak{G}}$ to $\operatorname{Mod}_{R}$ a local system of $R$-modules on $\mathfrak{G}$.

Definition 6.3. Let $R$ be a ring such that $-1 \neq 1$ in $R$. Let $\iota$ denote the generator of $\mathbb{Z} / 2 \mathbb{Z}$. An orientation functor of a category $\mathfrak{C}$ is a composition $\mathcal{R} \circ H$, where $H$ is a functor from $\mathfrak{C}$ to the category of $\mathbb{Z} / 2 \mathbb{Z}$-torsors, and $\mathcal{R}$ is the functor from the latter category to $\operatorname{Mod}_{R[\mathbb{Z} / 2 \mathbb{Z}]}$, which associated to a $\mathbb{Z} / 2 \mathbb{Z}$-torsor $Z$ the $R[\mathbb{Z} / 2 \mathbb{Z}]$-module $R[Z] /(1+\iota) R[Z]$.

An orientation local system $O$ of $\mathfrak{G}$ is a orientation functor $\mathcal{R} \circ H$ which is also a local system. When an element $u_{a} \in H(a)$ is chosen for each object $a$ of $\mathfrak{G}, O(h)$ may be identified with an element in $\{1,-1\}$ for each morphism $h$ in $\mathfrak{G}$. The fundamental triple is said to be orientable if it is possible to

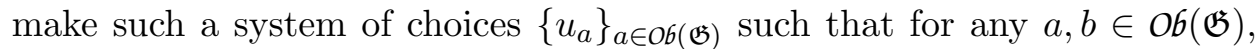
$H(h) u_{a}=u_{b}$ for all morphisms $h \in \mathcal{M o r}(\underline{\mathfrak{G})}$ from $a$ to $b$. Such a choice is said to be an orientation.

6.1.1. Floer theory package. Denote by $G_{\bullet}$ the groupoid of a single object $\bullet$, with $G$ as its set of morphisms.

A Floer theory in principle comes equipped with the following package:

[F1] a fundamental triple $\left\{\pi_{\mathfrak{G}}: \mathfrak{G} \rightarrow \underline{\mathfrak{G}}\right\}$,

[F2] a subset of $O \mathscr{G}(\mathfrak{G})$ consisting of "regular" elements; denote the associated subcategories of $\mathfrak{G}$ and $\underline{\mathfrak{G}}$ by $\mathfrak{G}^{\text {reg }}$ and $\underline{\mathfrak{G}}^{\text {reg }}$, respectively;

[F3] a functor $\mathcal{S}$ from $\mathfrak{G}^{\text {reg }}$ to $\mathbb{Z}_{\bullet}$, sending all objects to $\bullet$. This $\mathcal{S}$ factors as $\underline{\mathcal{S}} \circ \pi_{\mathfrak{G}}$. This is to define the notion of "spectral flow" or "relative $\mathbb{Z}$-grading,"

[F4] a parameter space $\mathcal{P}$ for the "flow equation" in the theory (such as of perturbations, metrics), so that corresponding to each $\mu \in \mathcal{P}$, there 
is a functor $\mathcal{E}_{\mu}$ from $\mathfrak{G}$ to $\mathbb{R}_{\bullet}$, sending all objects to $\bullet$. Each $\mathcal{E}_{\mu}$ factors as $\underline{\mathcal{E}}_{\mu} \circ \pi_{\mathfrak{G}}$. These are to be understood as notions of "action".

[F5] a subcategory $\mathfrak{C}_{\mu}$ of $\mathfrak{G}^{\text {reg }}$ defined for elements $\mu$ in a subset $\mathcal{P}^{\text {gen }} \subset \mathcal{P}$ consisting of "generic" elements, such that both $O b\left(\mathfrak{C}_{\mu}\right)$ and the set $\mathcal{M o r}_{1}\left(\mathfrak{C}_{\mu}\right):=\left\{z \mid z \in \mathcal{M} \operatorname{Mr}\left(\mathfrak{C}_{\mu}\right), \mathcal{S}(z)=1\right\}$ consist of countably many elements. Intuitively, $\mathfrak{C}_{\mu}$ represents the category of "critical points" and "broken trajectories" of the gradient flow of the actional functional parametrized by $\mu$.

Suppose $R$ is not of characteristic 2, but $R$ and $O$ are as in Definition 6.3 above. One needs additionally:

[F6] an orientation of $O$ for the fundamental triple $\pi_{\mathfrak{G}}: \mathfrak{G} \rightarrow \underline{\mathfrak{G}}$, which induces an orientation functor $O_{\mu}$ of $\mathfrak{C}_{\mu}$ for every $\mu \in \mathcal{P}$ gen .

As previously explained, by choosing an element $u_{a} \in H(a)$ for each $a \in$ $O b\left(\mathfrak{C}_{\mu}\right)$, one may identify $O_{\mu}(h)$ with a $\operatorname{sign}(z) \in\{-1,1\}$, for each morphism $z$ in $\mathfrak{C}_{\mu}$. This system of choice is called a coherent orientation for $\mathfrak{C}_{\mu}$. When $\pi_{\mathfrak{G}}: \mathfrak{G} \rightarrow \underline{\mathfrak{G}}$ comes from a (real) manifold $M$ and $\mathfrak{C}_{\mu}$ the category of critical points and broken trajectories of a Morse function on it, $O$ is defined from $\operatorname{det} T M$, and $O_{\mu}$ is defined from $\left\{\operatorname{det} T_{a} \mathcal{D}_{a}\right\}_{a \in O G\left(\mathfrak{C}_{\mu}\right)}$, where $\mathcal{D}_{a}$ denotes the descending manifold from the critical point $a$.

Note that by definition, $\underline{\mathfrak{G}}$ is groupoid; hence each $\mathcal{M o r}(\underline{\mathfrak{G}} ; a, b)$ may be identified with a group $\Pi$ after choosing a reference element in it. With respect to this choice, $\underline{\mathcal{S}}$ and $\underline{\mathcal{E}}_{\mu}$ identify, respectively, with the homomorphisms

$$
s: \Pi \rightarrow \mathbb{Z} \quad \text { and } \quad e_{\mu}: \Pi \rightarrow \mathbb{R} .
$$

6.1.2. Floer (co)homology with local coefficients. Given a Floer theory package described above and a local system, $\Lambda$, of $R$-modules on $\mathfrak{G}$, a purported Floer chain complex with local coefficient $\Lambda$ is defined from $\mathfrak{C}_{\mu}$ by the following chain groups and differentials:

$$
\begin{aligned}
C_{*}(\mathfrak{G}, \mu ; \Lambda) & =\bigoplus_{a \in O \in G\left(\mathfrak{C}_{\mu}\right)} O_{\mu}(a) \otimes \Lambda(a), \\
\partial & =\sum_{z \in \operatorname{Mor}_{1}\left(\mathfrak{C}_{\mu}\right),} O_{\mu}(z) \otimes \Lambda\left(\pi_{\mathfrak{G}} z\right),
\end{aligned}
$$

Since $\mathcal{S}(h)-\mathcal{S}\left(h^{\prime}\right)=s\left(h-h^{\prime}\right)$, where $h, h^{\prime} \in \mathcal{M}$ or $(\underline{\mathfrak{G}} ; a, b)$ and hence $h-h^{\prime} \in$ $\Pi$, the functor $\mathcal{S}$ defines a $\mathbb{Z} / p \mathbb{Z}$-grading on the Floer complex by

$$
\operatorname{gr}(a, b)=\mathcal{S}(h) \bmod p \quad \text { for any } h \in \mathfrak{M o r}(\underline{\mathfrak{G}} ; a, b),
$$

where $p$ is the divisibility of $s$.

The Floer cochain complex is obtained by replacing the category $\mathfrak{G}$ above by $\mathfrak{G}^{o p}$.

Let $\mathcal{M o r}_{1}\left(\mathfrak{C}_{\mu} ; a, b\right)$ denote the set consisting of elements in $\mathcal{M o r}_{1}\left(\mathfrak{C}_{\mu}\right)$ which go from the object $a$ to the object $b$, and let $\mathcal{M o r}_{1, h}\left(\mathfrak{C}_{\mu} ; a, b\right)$ be its subset 
consisting of elements in the "relative homotopy class" $h \in \mathcal{M} \operatorname{Mor}(\underline{\mathfrak{G}} ; a, b)$. Let $\mathcal{M o r}_{1}(\underline{\mathfrak{G}} ; a, b)=\{h \in \mathcal{M o r}(\underline{\mathfrak{G}} ; a, b) \mid \underline{\mathcal{S}}(h)=1\}$. The chain groups and differentials given can be written more concretely once a coherent orientation of $\mathfrak{C}_{\mu}$ is chosen:

$$
\begin{aligned}
C_{*}(\mathfrak{G}, \mu ; \Lambda) & =\bigoplus_{a \in \mathcal{O}\left(\mathfrak{C}_{\mu}\right)} \Lambda(a), \\
\partial a & =\sum_{b \in \mathcal{O G}\left(\mathfrak{C}_{\mu}\right)} \sum_{h \in \mathfrak{M o r}_{1}(\underline{\mathfrak{G}} ; a, b)} \sigma_{h}(a, b) \Lambda(h) b, \quad \text { where } \\
\sigma_{h}(a, b) & =\sum_{z \in \mathcal{M o r}_{1, h}\left(\mathfrak{C}_{\mu} ; a, b\right),} \operatorname{sign}(z) .
\end{aligned}
$$

In order for the map $\partial$ to be well-defined, certain bounds on the number of elements in $\mathcal{M o r}_{1}\left(\mathfrak{C}_{\mu} ; a, b\right)$ are required in order to make sense of the formula for $\partial$ above. These are typically provided by results of the following form:

For a generic $\mu$, the number of elements in $\mathcal{M o r}_{1}^{L}\left(\mathfrak{C}_{\mu} ; a, b\right)$ is finite for any $L \in \mathbb{R}$,

where $\mathcal{M o r}_{1}^{L}\left(\mathfrak{C}_{\mu} ; a, b\right)=\left\{z \mid z \in \mathcal{M o r}_{1}\left(\mathfrak{C}_{\mu} ; a, b\right), \mathcal{E}_{\mu}(z) \leq L\right\}$.

Since $\mathcal{E}_{\mu}$ factors through $\pi_{\mathfrak{G}}$, this guarantees the finiteness of $\sigma_{h}(a, b)$ for each triple of $a, b, h$. However, $\mathcal{M o r}_{1}(\underline{\mathfrak{G}} ; a, b)$ often has infinitely many elements: with a choice a reference element, it identifies with Ker $\mathcal{s}$. Nevertheless, together with the following additional condition, (6.3) guarantees that $\sigma_{h}(a, b)=0$ except for only finitely many elements $h$ in $\mathcal{M o r}_{1}(\underline{\mathfrak{G}} ; a, b)$; hence the formula for $\partial$ is $(6.2)$ is a finite sum when $O b\left(\mathfrak{C}_{\mu}\right)$ has finitely many elements: $\left.e_{\mu}\right|_{\text {Ker } s}=0$, or equivalently,

$$
e_{\mu}=-\kappa s \text { for some } \kappa \in \mathbb{R} \text { as elements in } \operatorname{Hom}(\Pi, \mathbb{R}) .
$$

When (6.4) fails, the infinite sum in the second line of (6.2) may be made sense of via particular choices of the local coefficients $\Lambda$, as follows.

Let $L \in \mathbb{R}$ from this point on. Paraphrasing Definitions 30.2.1 and 30.2.2 in $[\mathbf{K M}]$, we say that:

Definition 6.4. subset $S \subset \mathcal{M o r}(\underline{\mathfrak{G}} ; a, b)$ is $\left(s, e_{\mu}\right)$-finite if:

(i) for all $L$, the intersection $S \cap \mathcal{M o r}^{L}(\underline{\mathfrak{G}} ; a, b)$ is finite.

(ii) there exists $d$ such that $\mathcal{S}(h) \leq d$ for all $h \in S$.

A local system of complete, filtered topological abelian groups $\Lambda$ on $\mathfrak{G}$ is $\left(s, e_{\mu}\right)$-complete if, for any $a, b \in O \mathscr{G}(\mathfrak{G})$ and any $\left(s, e_{\mu}\right)$-finite set $S \subset$ $\operatorname{Mor}(\underline{\mathfrak{G}} ; a, b)$,

(i) the set $\{\Lambda(h) \mid h \in S\} \subset \operatorname{Hom}(\Lambda(a), \Lambda(b))$ is equicontinuous;

(ii) the homomorphisms $\Lambda(h)$ converges to 0 as $h$ runs through $S$.

In the above, $\operatorname{Hom}(\Lambda(a), \Lambda(b))$ is equipped with the compact-open topology, and the notions of complete filtered groups and equicontinuity are as 
explained in pp. 601-602 in [KM]. Note that the above definitions depend only on $\left(s, e_{\mu}\right)$, not on the particular choices of $\mathcal{S}$ and $\mathcal{E}_{\mu}$, and a local system is $\left(s, e_{\mu}\right)$-complete if and only if it is $\left(s, \lambda e_{\mu}\right)$-complete, for any $\lambda \in \mathbb{R}^{+}$.

As was observed in $[\mathbf{K M}]$, the upshot of this definition is that for any function $\sigma: \operatorname{Mor}(\underline{\mathfrak{G}} ; a, b) \rightarrow \mathbb{Z}$ with $\left(s, e_{\mu}\right)$-finite support, a series of the form $\sum_{h} \sigma(h) \Lambda(h)$ converges to a continuous limit, and the terms of this series may be rearranged. Apply this observation to the series $\sum_{h \in M_{\text {or }_{1}(\mathfrak{G} ; a, b)}} \sigma_{h}(a, b)$. The statement (6.3) implies that the function $h \mapsto$ $\sigma_{h}(a, b)$ is supported on a $\left(s, e_{\mu}\right)$-finite set, and hence the convergence of this series. In sum, the formula (6.2) gives a valid definition of Floer homology of local coefficient $\Lambda$, once the following two conditions are met:

$$
\left\{\begin{array}{l}
\bullet \text { there are finitely many elements in } \mathfrak{C}_{\mu} ; \\
\bullet \text { the statement }(6.3) \text { holds. }
\end{array}\right.
$$

Example. In the case of (6.4), any (local) coefficient is $\left(s, e_{\mu}\right)$-complete. In particular, Floer homology with coefficient $\mathbb{Z}$ is well-defined. The monotone cases discussed in Sections 1.1 and 1.2 belong to this case with $\kappa \neq 0$. (The $\kappa=0$ case is the "balanced" case in the terminology of $[\mathbf{K M}]$.)

6.2. The non-monotone case. As explained in the previous subsection, the periodic Floer homology and Seiberg-Witten cohomology can be defined in general with appropriate choice of local coefficients.

6.2.1. General Periodic Floer homology. Fix a volume form $w_{F}$ on $F$. In the terminology of the previous subsection, this Floer theory associates the following to each symplectic isotopy class of $f$ and $\Gamma \in H_{1}(M ; \mathbb{Z})$ :

- One may take $\mathfrak{G}$ to be the category with integral one-cycles in $M$ in the class of $\Gamma$ as objects, and with $\mathcal{M o r}\left(\mathfrak{G} ; \Theta, \Theta^{\prime}\right)$ consisting of integral 2-cycles $\Sigma$ in $\mathbb{R} \times M$, which limit to $\Theta$ or $\Theta^{\prime}$, respectively, as $s \rightarrow \infty$ or $s \rightarrow-\infty$. Let $\underline{\mathfrak{G}}$ be the category with $\mathcal{M o r}\left(\underline{\mathfrak{G}} ; \Theta, \Theta^{\prime}\right)=H_{2}\left(M ; \Theta, \Theta^{\prime}\right)$, and let $\pi_{\mathfrak{G}}$ be the functor which sends an integral one-cycle $\Theta \subset M$ to itself, and an integral two-cycle $\Sigma \subset \mathbb{R} \times M$ described above to the relative homology class of its projection to $M$.

- The functor $\mathcal{S}$ in this context is given by the relative grading $I\left(\Theta_{+}, \Theta_{-} ; Z\right)$ described in Section 1.1.

- The parameter space $\mathcal{P}$ may be taken to be the space of pairs $(f, J)$, where $f$ is a symplectomorphism in a fixed Hamiltonian isotopy class, and $J \in \mathcal{J}_{f}$. Using the same notation to denote the pullback of $w_{f}$ under the projection $\mathbb{R} \times M \rightarrow M$, set

$$
\mathcal{E}_{\mu}(\Sigma)=\int_{\Sigma} w_{f}, \quad \text { for } \Sigma \in \mathcal{M o r}(\mathfrak{G})
$$


- We call a $\mu=(f, J) \in \mathcal{P}$ "generic" in this context if $f$ is nondegenerate, and $J \in \mathcal{J}_{1 f}$. Let $\mathfrak{C}_{\mu}$ be the category with $06\left(\mathfrak{C}_{\mu}\right)=\mathcal{A}$ and with $\mathcal{M o r}\left(\mathfrak{C}_{\mu}\right)$ consisting of what is termed "broken GFL"s in Definition 9.3 of $[\mathbf{H 1}]$. By Lemma 9.3 in the same reference and its generalization in Section 9.5, the set $\mathcal{M o r}_{1}\left(\mathfrak{C}_{\mu} ; \Theta_{+}, \Theta_{-}\right)$is the set $\mathcal{M}_{1}\left(\Theta_{+}, \Theta_{-}\right) / \mathbb{R}$ previously introduced in Section 1.1.

- It is explained in Section 9 of [HT1] that a coherent orientation is provided by a choice of ordering of the positive hyperbolic periodic orbits in $\Theta$ for each $\Theta \in \mathcal{A}$.

In fact, the definitions of $\mathfrak{G}$ and $\mathcal{P}$ above may be chosen somewhat differently, since they do not directly enter into the definition of the Floer complex. Note also that in this Floer theory package,

$$
\Pi=H_{2}(M ; \mathbb{Z}), \quad s=c_{\Gamma}, \quad e_{\mu}=\left[w_{f}\right],
$$

regarding the latter two as homomorphisms from $H_{2}(M ; \mathbb{Z})$ to $\mathbb{Z}$ and $\mathbb{R}$, respectively. The condition (6.4) becomes the definition for monotone classes in Section 1.1: The cases $\kappa>0$ and $\kappa<0$ correspond to the positive monotone and negative monotone cases, respectively, and $\kappa \neq 0$ in this context.

With $\mathfrak{C}_{\mu}$ and $\mathcal{E}_{\mu}$ thus specified, the validity of the statement (6.3) follows from the typical Gromov compactness arguments as those which appeared in Lemma 9.8 of $[\mathbf{H 1}]$. It is also noted in Section 1.1 that $\mathcal{A}$ consists of finitely many elements. Thus, as both conditions in (6.5) are met, given a $\left(c_{\Gamma},\left[w_{f}\right]\right)$-complete local coefficient $\Lambda$ on $\mathfrak{G}$, the formula (6.1) gives a welldefined chain complex. We call the associated homology the periodic Floer homology with coefficient $\Lambda$ of the parameter set $\boldsymbol{\mu}_{P}=\left\{\left(F, w_{F}\right), f, \Gamma, J\right\}$, and denote it by

$$
H P_{*}\left(f:\left(F, w_{F}\right) \circlearrowleft, \Gamma ; \Lambda\right)_{J},
$$

or simply $H P_{*}(f, \Gamma ; \Lambda)$ when there is no danger of ambiguity. Under the monotonicity assumption, $\Lambda$ can be taken to be $\mathbb{Z}$; in this case the above definition of periodic Floer homology reduces to the version in Section 1.1.

6.2.2. General Seiberg-Witten cohomology. In this Floer theory, choose

- $\mathfrak{G}$ such that its objects consist of gauge equivalence classes of configurations on $M$, and such that $\mathcal{M o r}\left(\mathfrak{G} ; \mathfrak{c}_{-}, \mathfrak{c}_{+}\right)=\mathcal{B}\left(\mathfrak{c}_{-}, \mathfrak{c}_{+}\right)$. Let $\underline{\mathfrak{G}}$ be the category with the same objects, and with $\mathcal{M o r}\left(\underline{\mathfrak{G}} ; \mathfrak{c}_{-}, \mathfrak{c}_{+}\right)=$ $\pi_{0}\left(\mathcal{B}\left(\mathfrak{c}_{-}, \mathfrak{c}_{+}\right)\right)$. Let $\pi_{\mathfrak{G}}$ be defined by taking the relative homotopy classes of elements in $\mathcal{B}\left(\mathfrak{c}_{-}, \mathfrak{c}_{+}\right)$.

- Define $\mathcal{S}$ using the relative grading $\mathfrak{I}\left(\mathfrak{c}_{-}, \mathfrak{c}_{+} ; \mathfrak{h}\right)$ introduced in Section 1.2 .

- Take the parameter space $\mathcal{P}$ to be the set of triples $\mu=(\varpi, g,(\mathfrak{T}, \mathfrak{S}))$. Assume through out this article that $[\varpi] \neq 2 \pi c_{1}(\mathfrak{s})$ in $H^{2}(M ; \mathbb{R})$. 
Choose $\varepsilon_{\mu}$ such that $\varepsilon_{\mu}(\gamma)=\frac{1}{2} \varepsilon_{\omega, \mathfrak{q}}^{\mathrm{top}}(\gamma)$ in the notation of $[\mathbf{K M}]$, where $\omega=-\mathrm{i} \varpi / 4, \mathfrak{q}=(\mathfrak{T}, \mathfrak{S})$.

- When $\mu$ is generic in the sense explained in Section 1.2, let $\mathfrak{C}_{\mu}$ be the category with $\mathcal{O b}\left(\mathfrak{C}_{\mu}\right)=\mathcal{C}$, and with $\mathcal{M o r}\left(\mathfrak{C}_{\mu} ; \mathfrak{c}_{-}, \mathfrak{c}_{+}\right)=\breve{M}^{+}\left(\mathfrak{c}_{-}, \mathfrak{c}_{+}\right)$ in the notation of $[\mathbf{K M}]$. In particular, $\mathcal{M o r}_{1}\left(\mathfrak{C}_{\mu} ; \mathfrak{c}_{-}, \mathfrak{c}_{+}\right)$is what is denoted by $\mathcal{M}_{1}\left(\mathfrak{c}_{-}, \mathfrak{c}_{+}\right) / \mathbb{R}$ in Section 1.1.

- The functor $O_{\mu}$ assigns to each $\mathfrak{c} \in \mathrm{e}^{r}$ the module in (22.1) of $[\mathbf{K M}]$ (the two element set $\Lambda$ in $[\mathbf{K M}]$ is $H$ in our notation), and to each $\gamma \in \mathcal{M}_{1}\left(\mathfrak{c}_{-}, \mathfrak{c}_{+}\right) / \mathbb{R}$, the isomorphism $\epsilon(\gamma)$ in $(22.4)$ of $[\mathbf{K M}]$.

By the above choice of $\mathcal{S}, \mathcal{E}_{\mu}$ and the discussion in Section 1.1, in this Floer theory

$$
\Pi=H^{1}(M ; \mathbb{Z}), \quad s=c_{1}(\mathfrak{s}), \quad e_{\mu}=p_{\mathfrak{s}, \varpi} .
$$

The condition (6.4) now corresponds to the positive monotone, negative monotone, and balanced cases discussed in Chapter 29 of $[\mathbf{K M}]$ when $\kappa<0$, $\kappa>0$, and $\kappa=0$, respectively.

It is shown in $[\mathbf{K M}]$ that the conditions (1.12) and (6.5) hold under our assumption that $p_{\mathfrak{s}, \varpi} \neq 0$, and thus for any $\left(c_{1}(\mathfrak{s}), p_{\mathfrak{s}, \varpi}\right)$-complete local system $\Lambda$, the construction in the previous subsection defines a Floer homology with coefficient $\Lambda$ associated to each parameter set $\boldsymbol{\mu}_{S}=\{M, \mathfrak{s}, \varpi, g, \mathfrak{q}=$ $(\mathfrak{T}, \mathfrak{S})\}$. As previously observed, by replacing $\mathfrak{G}$ with $\mathfrak{G}^{o p}$, the same construction defines a Floer cohomology. Thus, for any $\left(c_{1}(\mathfrak{s}),-p_{\mathfrak{s}, \varpi}\right)$-complete local system $\Lambda$, there is a well-defined Floer cochain complex:

$$
\begin{aligned}
C^{*}(\mathfrak{G}, \mu ; \Lambda) & =\bigoplus_{a \in O G\left(\mathfrak{C}_{\mu}\right)} O_{\mu}(a) \otimes \Lambda(a), \\
\delta & =\sum_{z \in \mathfrak{M o r}_{1}\left(\mathfrak{C}_{\mu}\right)} O_{\mu}\left(z^{-1}\right) \otimes \Lambda\left(\pi_{\mathfrak{G}} z^{-1}\right) .
\end{aligned}
$$

The resulting cohomology is the Seiberg-Witten-Floer cohomology with coefficient $\Lambda$ for $\boldsymbol{\mu}_{S}$. This Floer cohomology only depends on the data set (1.17). We denote it by $\operatorname{HM}^{*}\left(M, \mathfrak{s}, p_{\mathfrak{s}, \varpi} ; \Lambda\right)$. To compare with the notation in $[\mathbf{K M}]$,

$$
H M^{*}(M, \mathfrak{s},-\pi[\varpi] ; \Lambda)=\operatorname{HM}^{*}\left(M, \mathfrak{s}, p_{\mathfrak{s}, \varpi} ; \Lambda\right),
$$

and what we call a $\left(c_{1}(\mathfrak{s}),-p_{\mathfrak{s}, \varpi}\right)$-complete local system is in the terminology of $[\mathbf{K M}]$, a $c$-dual complete local system with $c=\pi[\varpi]$. When $\mathfrak{s}, \varpi$ are monotone with respect to each other, the coefficient $\Lambda$ will often be dropped from the notation when it is set to be $\mathbb{Z}$.

6.3. The general isomorphism theorem. We now generalize Theorem 1.1 to include the non-monotone cases. Associate to each periodic Floer homology data set $\boldsymbol{\mu}_{P}$ a Seiberg-Witten-Floer cohomology data set $\boldsymbol{\mu}_{S}$, as described in Section 1.3. 
Fix $L \geq 1$. Given a pair, $\Theta_{-}$and $\Theta_{+}$, from $\mathcal{A}$, introduce $\mathcal{M}_{1 L}\left(\Theta_{+}, \Theta_{-}\right) \subset$ $\mathcal{M}_{1}\left(\Theta_{+}, \Theta_{-}\right)$to denote the elements $\Sigma$ such that

$$
\int_{C} w_{f} \leq L
$$

where $C$ is the sole submanifold from $\Sigma$ that is not an $\mathbb{R}$-invariant cylinder. The space $\mathcal{M}_{1 L}\left(\Theta_{+}, \Theta_{-}\right)$has a finite set of components. This is asserted by Theorem 1.8 in $[\mathbf{H 1}]$. A value for $L$ in $[1, \infty)$ is said to be generic when there are no cases where (6.8) is an equality. The set of generic values is necessarily the complement of a countable set.

As will be seen momentarily, the space $\mathcal{M}_{1 L}\left(\Theta_{+}, \Theta_{-}\right)$has a precise analog on the Seiberg-Witten side of the ledger. To define this analog, fix $r \geq 1$ such that each solution to (3.13) is non-degenerate. Suppose that $\mathfrak{c}_{-}$and $\mathfrak{c}_{+}$are elements in $\mathrm{C}^{r}$. Given $L \geq 1$, use $\mathcal{M}_{1 L}\left(\mathfrak{c}_{-}, \mathfrak{c}_{+}\right) \subset \mathcal{M}_{1}\left(\mathfrak{c}_{-}, \mathfrak{c}_{+}\right)$to denote the subspace of instantons $\mathfrak{d}=(A, \psi)$ that obey (5.8), namely

$$
\frac{\mathrm{i}}{2 \pi} \int_{\mathbb{R} \times M} \frac{\partial A}{\partial s} d s \wedge w_{f} \leq L .
$$

Note in this regard that the above integral is absolutely convergent given that $\mathfrak{c}_{-}$and $\mathfrak{c}_{+}$are both non-degenerate.

Recall from Section 3 that Theorem 3.1 makes no assumption on monotonicity. Thus, it constructs an isomorphism $\Phi^{r}$ from $\mathcal{A}$ to $\mathrm{e}^{r}$. Keep in mind that both consist of finitely many non-degenerate elements. Let $\Theta_{-}$and $\Theta_{+}$ denote two elements in $\mathcal{A}$, and let $\mathfrak{c}_{-}$and $\mathfrak{c}_{+}$denote solutions to (3.11) that give $\Phi^{r}\left(\Theta_{-}\right)$and $\Phi^{r}\left(\Theta_{+}\right)$. Theorem 4.1 as stated does require the assumption of monotonicity to guarantee that both $\mathcal{M}_{1}\left(\Theta_{+}, \Theta_{-}\right)$and $\mathcal{M}_{1}\left(\mathfrak{c}_{-}, \mathfrak{c}_{+}\right)$ are finite. However, the theorem that follows is the non-monotone analog.

Theorem 6.1. Let $\boldsymbol{\mu}_{P}=\left\{\left(F, w_{F}\right), f, \Gamma, J\right\}$ and $\boldsymbol{\mu}_{S}=\left\{M, \mathfrak{s}_{\Gamma}, \varpi_{r}, g, \mathfrak{q}\right\}$ be as in the statement of Theorem 1.1. Fix a generic $L \geq 1$. Then there exists a constant $\kappa_{L}>1$ such that given $N>\kappa_{L}, \delta<\kappa_{L}^{-1}$ and $a(\delta, N)$ approximation $\left(f^{\prime}, J^{\prime}\right)$ to $(f, J)$, there exists $\kappa_{L}^{\prime} \geq \kappa_{L}$ so that for any $r \geq \kappa_{L}^{\prime}$, the following hold for $\mathcal{A}$ and $\mathrm{e}^{r}$ defined using $\left(f^{\prime}, J^{\prime}\right)$ :

(0) Theorem 3.1 can be invoked so as to provide the identification, $\Phi^{r}$, between $\mathcal{A}$ and $\mathrm{e}^{r}$.

(1) Fix a pair $\Theta_{-}$and $\Theta_{+}$in $\mathcal{A}$; and let $\mathfrak{c}_{-}$and $\mathfrak{c}_{+}$denote solutions to (3.13) whose respective gauge equivalence classes are $\Phi^{r}\left(\Theta_{-}\right)$and $\Phi^{r}\left(\Theta_{+}\right)$. Let $\mathcal{M}_{1 L}\left(\Theta_{+}, \Theta_{-}\right)$and $\mathcal{M}_{1 L}\left(\mathfrak{c}_{-}, \mathfrak{c}_{+}\right)$be the versions defined using $\left(f^{\prime}, J^{\prime}\right)$.

(a) The space $\mathcal{M}_{1 L}\left(\mathfrak{c}_{-}, \mathfrak{c}_{+}\right)$has a finite set of components and each component consists of non-degenerate solutions to (4.4).

(b) There is a smooth $\mathbb{R}$-equivariant diffeomorphism

$$
\Psi^{r}: \mathcal{M}_{1 L}\left(\Theta_{+}, \Theta_{-}\right) \rightarrow \mathcal{M}_{1 L}\left(\mathfrak{c}_{-}, \mathfrak{c}_{+}\right) .
$$


(2) There are coherent systems of orientations for sheaves $\left\{\Lambda\left(\Theta_{+}, \Theta_{-}\right)\right\}_{\Theta_{-}, \Theta_{+} \in \mathcal{A}}$ and $\left\{\Lambda\left(\mathfrak{c}_{-}, \mathfrak{c}_{+}\right)\right\}_{\mathfrak{c}_{-}, \mathfrak{c}_{+} \in \mathcal{C}^{r}}$ such that any given $\left(\Theta_{-}, \Theta_{+}\right)$version of $\Psi^{r}$ maps the orientation $\mathfrak{o}_{p F h}$ to the orientation $\mathfrak{o}_{S W}$.

Proof. The arguments used to prove Theorem 4.1 can be employed with only cosmetic changes to prove the following: Fix a generic $L \geq 1$. Then there exists an $L$-dependent constant $\kappa_{L}$ such that if $r \geq \kappa_{L}$, the conclusions of assertions (1) and (2) hold with $\mathcal{M}_{1}\left(\Theta_{+}, \Theta_{-}\right)$and $\mathcal{M}_{1}\left(\mathfrak{c}_{-}, \mathfrak{c}_{+}\right)$replaced, respectively, by $\mathcal{M}_{1 L}\left(\Theta_{+}, \Theta_{-}\right)$and $\mathcal{M}_{1 L}\left(\mathfrak{c}_{-}, \mathfrak{c}_{+}\right)$. Indeed, the only change to Part 1 is the change from $\mathcal{M}_{1}(\cdot)$ to $\mathcal{M}_{1 L}(\cdot)$. The gluing construction used in the proof shows that $\Psi^{r}$ maps $\mathcal{M}_{1 L}\left(\Theta_{+}, \Theta_{-}\right)$injectively into $\mathcal{M}_{1 L}\left(\mathfrak{c}_{-}, \mathfrak{c}_{+}\right)$ when $r$ is greater than a purely $L$-dependent constant. The proof that Theorem 4.1's version of $\Psi^{r}$ maps $\mathcal{M}_{1 L}\left(\Theta_{+}, \Theta_{-}\right)$surjectively onto $\mathcal{M}_{1 L}\left(\mathfrak{c}_{-}, \mathfrak{c}_{+}\right)$in the monotone case uses the monotonicity assumption only to obtain a bound on (4.7) from a bound on the index. The bound on (4.7) is then used to derive the global results in Section 5. In the case at hand, the bound on (4.7) is assumed because we are restricting attention to instantons in $\mathcal{M}_{1 L}\left(\mathfrak{c}_{-}, \mathfrak{c}_{+}\right)$. This understood, the arguments in Section 5 can be used verbatim to prove that $\Psi^{r}$ maps $\mathcal{M}_{1 L}\left(\Theta_{+}, \Theta_{-}\right)$surjectively onto $\mathcal{M}_{1 L}\left(\mathfrak{c}_{-}, \mathfrak{c}_{+}\right)$.

Noting that the conditions (5.8) and (6.9) correspond under $\Psi^{r}$, straightforward modifications of the direct limit arguments in Sections $4 \mathrm{~d}-4 \mathrm{i}$ of [T1] then yield the following:

Theorem 6.2. Let $\boldsymbol{\mu}_{P}=\left\{\left(F, w_{F}\right), f, \Gamma, J\right\}$ be a periodic Floer parameter set, where $\Gamma \in H_{1}(M ; \mathbb{Z})$ is an arbitrary class. Associate to it a Seiberg-Witten parameter set $\boldsymbol{\mu}_{S}=\left\{M, \mathfrak{s}_{\Gamma}, \varpi_{r}, g, \mathfrak{q}\right\}$ according to the recipe described in Section 1.3. Let $\Lambda_{P}$ be a $\left(c_{\Gamma},\left[w_{f}\right]\right)$-complete local system for the periodic Floer homology, and let $\Lambda_{S}$ denote a corresponding $\left(c_{1}\left(\mathfrak{s}_{\Gamma}\right), 2 r \pi\left[w_{f}\right]\right)$-complete local system for the Seiberg-Witten cohomology, in the sense specified below. Then there is an isomorphism between the two Floer (co)homologies with coefficient $\Lambda$

$$
H P_{*}\left(f:\left(F, w_{F}\right) \circlearrowleft, \Gamma ; \Lambda_{P}\right)_{J} \simeq \mathrm{HM}^{-*}\left(M, \mathfrak{s}_{\Gamma},-2 r \pi\left[w_{f}\right] ; \Lambda_{S}\right),
$$

which reverses the relative $\mathbb{Z} / p \mathbb{Z}$-gradings.

Use the subscripts $P$ and $S$, respectively, to label the ingredients $\pi_{\mathfrak{G}}$ : $\mathfrak{G} \rightarrow \underline{\mathfrak{G}}$, etc. in the periodic Floer theory and Seiberg-Witten-Floer theory, as described in the previous subsection. To introduce $\Lambda_{S}$, note that only its restriction to $\mathfrak{C}_{\mu_{S}}$ is relevant in the statement of the preceding theorem. A $\left(c_{\Gamma},\left[w_{f}\right]\right)$-complete local system $\Lambda_{P}$ on $\mathfrak{G}_{P}$ specifies a local system on this restriction via the maps $\Phi^{r}, \Psi^{r}$. In particular, recall from Section 1.3 that for a periodic Floer parameter set $\boldsymbol{\mu}_{P}$ and its corresponding Seiberg-Witten 
parameter set $\boldsymbol{\mu}_{S}$, one has $c_{\Gamma}=c_{\mathfrak{s}_{\Gamma}}$ and $p_{\mathfrak{s}, \varpi_{r}}=-2 r \pi\left[w_{f}\right]$. Moreover, comparing (6.6) and (6.7), and we see that the two Floer theories have identical $s$, while $e_{\mu}$ on the Seiberg-Witten side is $-2 r \pi$ times that on the periodic Floer homology side. Thus, $\Lambda_{P}$ is $\left(s, e_{\mu}\right)$-complete on the periodic Floer homology side if and only if $\Lambda_{S}$ is $\left(s,-e_{\mu}\right)$-complete on the Seiberg-Witten-Floer theory side. In other words, the Seiberg-Witten-Floer cohomology for $\boldsymbol{\mu}_{S}$ with coefficient $\Lambda_{S}$ is well-defined exactly when the periodic Floer homology for $\boldsymbol{\mu}_{P}$ with coefficient $\Lambda_{P}$ is.

Corollary 1.1 generalizes correspondingly:

Corollary 6.1. The general periodic Floer homology in the previous theorem depends only on the Hamiltonian isotopy class of $f$, the homology class $\Gamma$, and the local system $\Lambda$. In fact, the periodic Floer homology with "maximallytwisted coefficients" depends only on the symplectic isotopy class off and $\Gamma$, in the following sense:

Let $\Lambda_{\left[w_{f}\right]} \supset \mathbb{Z}\left[H_{1}(M ; \mathbb{Z})\right]$ denote the Novikov ring consisting of $\mathbb{Z}$-valued functions on $H_{1}(M ; \mathbb{Z})$ that are finitely supported on $\left[w_{f}\right]^{-1}(-\infty, \kappa]$ for any $\kappa \in \mathbb{R}$, regarding $\left[w_{f}\right]$ as an element in $\operatorname{Hom}\left(H_{2}(M ; \mathbb{Z}), \mathbb{R}\right)$. Then there is $a \mathbb{Z}\left[H_{1}(M ; \mathbb{Z})\right]$-module with relative $\mathbb{Z} / p \mathbb{Z}$-grading, denoted $H P_{*}^{[f]}(\Gamma)$, such that

$$
H P_{*}\left(f, \Gamma ; \Lambda_{\left[w_{f}\right]}\right) \simeq H P_{*}^{[f]}(\Gamma) \otimes_{\mathbb{Z}\left[H_{1}(M ; \mathbb{Z})\right]} \Lambda_{\left[w_{f}\right]},
$$

and $H P_{*}^{[f]}(\Gamma)$ depends only on the symplectic isotopy class $[f]$ and $\Gamma$.

Proof. Because of the previous Theorem, it suffices to check the corresponding statement for $\operatorname{HM}^{*}\left(M, \mathfrak{s}_{\Gamma},-2 r \pi\left[w_{f}\right] ; \Lambda_{\left[w_{f}\right]}\right)$. First, observe that

$$
\overline{\operatorname{HM}}^{*}\left(M, \mathfrak{s}_{\Gamma}, 0 ; \Lambda_{\left[w_{f}\right]}\right)=\overline{H M}^{*}\left(M, \mathfrak{s}_{\Gamma}, c_{b} ; \Lambda_{\left[w_{f}\right]}\right)=0,
$$

due to the well-known fact that the Novikov homology of a torus is trivial. Thus, we may again write $\widehat{\mathrm{HM}}^{*}\left(M, \mathfrak{s}_{\Gamma}, 0 ; \Lambda_{\left[w_{f}\right]}\right)=\widehat{\mathrm{HM}}^{*}\left(M, \mathfrak{s}_{\Gamma}, 0 ; \Lambda_{\left[w_{f}\right]}\right)=$ $\operatorname{HM}^{*}\left(M, \mathfrak{s}_{\Gamma}, 0 ; \Lambda_{\left[w_{f}\right]}\right)$. Now, the variant of Theorem 31.5.1 in $[\mathbf{K M}]$ with local coefficients states:

$$
\operatorname{HM}^{*}\left(M, \mathfrak{s}_{\Gamma}, 0 ; \Lambda_{\left[w_{f}\right]}\right) \simeq \operatorname{HM}^{*}\left(M, \mathfrak{s}_{\Gamma},-2 r \pi\left[w_{f}\right] ; \Lambda_{\left[w_{f}\right]}\right) .
$$

However, the coefficient ring $\mathbb{Z}\left[H_{1}(M ; \mathbb{Z})\right]$ is $\left(\mathfrak{s}_{\Gamma}, 0\right)$-complete, and $\Lambda_{\left[w_{f}\right]}$ is flat over $\mathbb{Z}\left[H_{1}(M ; \mathbb{Z})\right]$. Thus

$$
\operatorname{HM}^{*}\left(M, \mathfrak{s}_{\Gamma}, 0 ; \Lambda_{\left[w_{f}\right]}\right)=\operatorname{HM}^{*}\left(M, \mathfrak{s}_{\Gamma}, 0 ; \mathbb{Z}\left[H_{1}(M ; \mathbb{Z})\right]\right) \otimes_{\mathbb{Z}\left[H_{1}(M ; \mathbb{Z})\right]} \Lambda_{\left[w_{f}\right]} .
$$

For homologies classes $\Gamma$ with degree 1, the Periodic Floer homology reduces to the Floer homology of symplectic fixed points. Thus, in this special case, Corollaries 1.1, 6.1 reduce to the invariance properties of the latter Floer homology in the existent literature. 


\section{Appendices}

In Appendix 7.1, we describe the appropriate parameter space of the periodic Floer homology, and clarify its relation with the parameter space of the Seiberg-Witten-Floer cohomology. Note from the outset that in general, the coefficient system $\Lambda$ will not be considered to be among the parameters of a Floer theory since it is chosen extraneously. Moreover, it is clear from the discussion in Section 6 that local systems for two different Floer theories may be identified by identifying their respective versions of $\underline{\mathfrak{G}}$, which depend on the intrinsic parameters of the theory. So do the functions $s, e_{\mu}$ that determine the completeness condition of the local systems.

One application of the isomorphism theorem in this article is the computation of Seiberg-Witten-Floer cohomology for degree 1 Spin $^{c}$ structures of all mapping tori, via the computation of Floer homology of symplectic fixed points for oriented surfaces in the existent literature. We denote this latter Floer homology by $H F$. In Appendix 7.2, we describe the version of $H F$ which is equivalent to the periodic Floer homology. In $[\mathbf{C}]$ and its sequel, Cotton-Clay has a more refined definition of $H F$, which may be used to compute the version described below.

7.1. The dependence of $\boldsymbol{H P}$ on $f$. Let $h \in \operatorname{Symp}\left(F, w_{F}\right)$ be an element in the group of symplectomorphisms of $\left(F, w_{F}\right)$, and let $\operatorname{Symp}_{h}\left(F, w_{F}\right) \subset$ $\operatorname{Symp}\left(F, w_{F}\right)$ denote the path component containing $h$. We now define family versions of $M, w_{f}$, and $c_{1}\left(K^{-1}\right)$ parametrized by $\operatorname{Symp}_{h}\left(F, w_{F}\right)$ and its universal covering.

Let $\underline{M} \rightarrow \operatorname{Symp}_{h}\left(F, w_{F}\right)$ be defined as follows:

$$
\underline{M}=\left(\operatorname{Symp}_{h}\left(F, w_{F}\right) \times \mathbb{R} \times F\right) /((f, t, x) \sim(f, t+2 \pi, f(x))) .
$$

By projecting to the first factor of the product $\operatorname{Symp}_{h}\left(F, w_{F}\right) \times \mathbb{R} \times F, \underline{M}$ may be viewed as a bundle over $\operatorname{Symp}_{h}\left(F, w_{F}\right)$ whose fiber over $f$ is the mapping torus $M_{f}$ of $f$. By projecting to the first two factors, $\underline{M}$ may be viewed as a fiber bundle over $\operatorname{Symp}_{h}\left(F, w_{F}\right) \times S^{1}$; and the Euler class of the vertical tangent bundle $\underline{K}^{-1}$ over this fiber bundle, denoted by $\underline{c}$, restricts to the class $c_{1}\left(K^{-1}\right)$ on each $M_{f} \subset \underline{M}$. Finally, one may pull-back $w_{F}$ from the projection to the last factor $F$. This induces a closed two-form $\underline{w}$, which restricts to each $M_{f}$ as $w_{f}$.

Choose a connection $a$ on the bundle $\underline{M} \rightarrow \operatorname{Symp}_{h}\left(F, w_{F}\right)$ which preserves the structure of its fibers $M_{f}$ as a mapping torus of symplectomorphisms of $\left(F, w_{F}\right)$. Let $X$ denote a symplectic vector field on $\left(F, w_{F}\right)$ and thus an element in $T_{f} \operatorname{Symp}_{h}\left(F, w_{F}\right)$. A connection as just defined assigns to $X$ the 
following object:

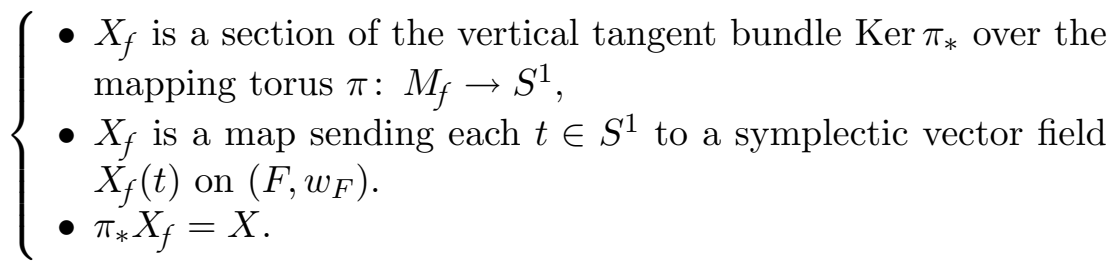

The preceding construction defines various associated bundles of $\underline{M} \rightarrow$ $\operatorname{Symp}_{h}\left(F, w_{F}\right)$ and connections on them. Note however that the monodromies of the various homology or cohomology bundles $H(M) \rightarrow$ $\underline{H}(M) \rightarrow \operatorname{Symp}_{h}\left(F, w_{F}\right)$ do not depend on the choice of connection on $\underline{M}$.

Let $\widetilde{M} \rightarrow \widetilde{\operatorname{Symp}}_{h}\left(F, w_{F}\right)$ be the pull-back bundle over $\underline{M}$ under the covering map of $\widetilde{\operatorname{Symp}}_{h}\left(F, w_{F}\right)$, and let $\widetilde{H}(M), \widetilde{c}, \widetilde{w}$, respectively denote the pull-backs of $\underline{H}(M), \underline{c}, \underline{w}$ over $\widetilde{M}$. The choice of connection on $\underline{M}$ above defines a trivialization of $\widetilde{M} \rightarrow \widetilde{\operatorname{Symp}}_{h}\left(F, w_{F}\right)$, which induces trivializations on its various associated bundles as well.

In order to compare with the Seiberg-Witten-Floer cohomology, we view the periodic Floer homology $H P$ as intrinsically an invariant of a fibered three-manifold $\pi: M \rightarrow S^{1}$, a homology class $\Gamma \in H_{1}(M ; \mathbb{Z})$, a closed twoform $w \in \Omega^{2}(M)$ which restricts to $w_{F}$ on the fibers, and an almost complex structure $J$ on $\mathbb{R} \times M$ satisfying the conditions (1.6), with $w_{f}$ therein replaced by $w$. From this point of view, $H P$ depends indirectly on the symplectomor$\operatorname{phism} f$ through the choice of the two-form $w=w_{f}$, and through the vector field $\partial_{t}$. Thus, to compare the periodic Floer homologies of different symplectomorphisms, first fix a lifting $f_{0} \in \widetilde{\operatorname{Symp}}_{h}\left(F, w_{F}\right)$ of $h$. Identify $M$ with the fiber $M_{f_{0}} \subset \widetilde{M}$, and set $w=w_{f_{0}}$. Then for each triple consisting of $\Gamma \in H_{1}\left(M_{f_{0}} ; \mathbb{Z}\right)$, a suitable local system $\Lambda$, and $J \in \mathcal{J}_{f_{0}}$, the construction in Sections 1 and 6 gives rise to a periodic Floer homology, which we denote by $H P\left(f_{0}, \Gamma ; \Lambda\right)_{J}$. For an arbitrary $f \in \widetilde{\operatorname{Symp}}_{h}\left(F, w_{F}\right)$, use the trivializations given by $a$ to define isomorphisms $\iota_{f}: M_{f} \rightarrow M_{f_{0}}, \iota_{f}^{*}: \Omega^{2}\left(M_{f_{0}}\right) \rightarrow \Omega^{2}\left(M_{f}\right)$, $\left(\iota_{f}\right)_{*}: H_{1}\left(M_{f}\right) \rightarrow H_{1}\left(M_{f_{0}}\right)$, and $\left(\iota_{f}\right)_{*}: \mathcal{J}_{f} \rightarrow \mathcal{J}_{f_{0}}$.

Then for each triple consisting of a $\Gamma_{f} \in H_{1}\left(M_{f}\right)$, a local system $\Lambda_{f}$ for $f$, and a $J_{f} \in \mathcal{J}_{f}$, we define $H P\left(f, \Gamma_{f} ; \Lambda_{f}\right)_{J_{f}}$ to be the periodic Floer homology associated to $\left(\iota_{f}\right)_{*} \Gamma,\left(\iota_{f}^{-1}\right)^{*} w_{f},\left(\iota_{f}^{-1}\right)^{*} \Lambda_{f}$, and $\left(\iota_{f}\right)_{*} J$. The completeness condition for $\Lambda_{f}$ is provided by pull-backs of $\left.\widetilde{c}\right|_{M_{f}}+2 e_{\Gamma_{f}}$ and $\left[w_{f}\right]$ via $\left(\iota_{f}^{-1}\right)^{*}$.

In this manner, we have defined the periodic Floer homology over the parameter space $\widetilde{H}_{1}(M ; \mathbb{Z}) \times{\widetilde{\operatorname{Symp}_{h}}}_{\mathcal{J}}$. Here, $\widetilde{\mathcal{J}}$ is the family version of $\mathcal{J}_{f}$ over $\widetilde{\operatorname{Symp}}_{h}=\widetilde{\operatorname{Symp}}_{h}\left(F, w_{F}\right)$. On the other hand, since the Seiberg-WittenFloer cohomology depends only on the $\operatorname{Spin}^{c}$ structure and the cohomology 
class of the perturbation two-form, one has $H M$ defined over the parameter space $\operatorname{Spin}^{c}(M) \times H^{2}(M ; \mathbb{Z})$, where $\operatorname{Spin}^{c}(M)$ denotes the set of $\operatorname{Spin}^{c}$ structures on $M$. Fix a sufficiently large $r$. The isomorphism $\Phi$ from $H P$ to $H M$ constructed in the main theorems of this article factors through a map $q$ between their parameter spaces, in the sense that $\Phi(H P(\cdot))=H M(q(\cdot))$. To describe this map $q$, first, note the aforementioned connection defines a trivialization

$$
\widetilde{H}_{1}(M ; \mathbb{Z}) \times{\widetilde{\operatorname{Symp}_{h}}}^{\mathcal{J}} \rightarrow H_{1}(M ; \mathbb{Z}) \times \widetilde{\operatorname{Symp}}_{h} \times \mathcal{J}_{f_{0}}
$$

The map $q$ is the composition of this with the map which sends $(\Gamma, f, J) \in$ $H_{1}(M ; \mathbb{Z}) \times \widehat{\operatorname{Symp}}_{h} \times \mathcal{J}_{f_{0}}$ to $\left(\mathfrak{s}_{\Gamma}, 2 r\left(\left[w_{f_{0}}\right]+6\left(\theta_{f}\right)\right)+2 \pi c_{\Gamma}\right) \in \operatorname{Spin}^{c}(M) \times$ $H^{2}(M ; \mathbb{R})$. In the above, $\mathfrak{s}_{\Gamma}$ is defined as in Section $1.3, \theta_{f}$ denotes the flux of a symplectic isotopy from $f_{0}$ to $f$, and the map $b$ is part of the Mayer-Vietoris sequence: $\cdots H^{1}(F ; \mathbb{R}) \stackrel{1-f^{*}}{\longrightarrow} H^{1}(F ; \mathbb{R}) \stackrel{6}{\rightarrow} H^{2}(M ; \mathbb{R}) \cdots$. As a consequence, we have a family of periodic Floer homologies parametrized by $\operatorname{Spin}^{c}(M) \times$ $H^{2}(M ; \mathbb{R})$, and this does not depend on the choice of connection on $\underline{M}$. When $f$ vary through a path in $\widetilde{\operatorname{Symp}}_{h}$ connecting two different lifts of the same symplectomorphism, the corresponding Spin $^{c}$-structure (determined by the vector field $\partial_{t}$ ) and the class $\left[w_{f}\right]$ will change simultaneously. The periodic Floer homology, and hence the corresponding Seiberg-Witten-Floer cohomology, parametrized by these two elements in $\operatorname{Spin}^{c}(M) \times H^{2}(M ; \mathbb{R})$ will however be isomorphic.

Remark. Cotton-Clay pointed out to us that there is no such monodromy unless $g=1$; therefore the above observation is interesting only in very limited cases.

Appendix 7.2: Relation with the Floer homology of fixed points. When $\Gamma$ has degree 1, a generator of $C P$ may be regarded as a section of $\pi: M \rightarrow S^{1}$, and by $[\mathbf{H 1}]$, an element in $\mathcal{M}_{1}\left(\Theta_{+}, \Theta_{-}\right)$used to define the differential of the periodic Floer chain complex consists of a holomorphic cylinder in $\mathbb{R} \times M$. When $J$ is admissible, such a cylinder may be viewed as a holomorphic section $\mathbb{R} \times(\mathbb{R} /(t \sim t+2 \pi) \rightarrow \mathbb{R} \times((\mathbb{R} \times F) / \sim)=\mathbb{R} \times M$. A section of $\pi: M \rightarrow S^{1}$ is by definition an element in the $f$-twisted loop space of $F$, which we denote by $\mathcal{L}_{f}$. A section of $\mathbb{R} \times M \rightarrow \mathbb{R} \times S^{1}$ can be interpreted as a path in $\mathcal{L}_{f}$. Given an element $\Gamma \in H_{1}(M ; \mathbb{Z})$, let $\mathcal{L}_{f, \Gamma}$ denote the space of sections of $\pi: M \rightarrow S^{1}$ in the homology class $\Gamma$. The $f$-twisted loop space decomposes as $\mathcal{L}_{f}=\coprod_{\Gamma \in \mathcal{N}} \mathcal{L}_{f, \Gamma}$, where $\mathcal{N}$ denotes the set of elements $\Gamma \in H_{1}(M ; \mathbb{Z})$ with $\left\langle e_{\Gamma^{*}},[F]\right\rangle=1$. This is an affine space under $H_{1}(F ; \mathbb{Z}) /\left(1-f_{*}\right)$. 
For lack of space, we shall only outline the construction of $H F$, and refer the reader to Sections 2 and 3 of $[\mathbf{L 2}]$ for more details.

Fix the symplectic structure $\left(F, w_{F}\right)$. The space of admissible almost complex structures on $\mathbb{R} \times M$ is identical with the space $\mathcal{J}_{K}$ in $[\mathbf{L 2}]$, and an element from this space defines a metric on $\mathcal{L}_{f}$. Let $X_{f}$ be the space of elements satisfying the first two conditions of (7.1). Given an $X_{f} \in X_{f}$, equation (13) of $[\mathbf{L 2}]$ defines an action one-form on $\mathcal{L}_{f}$. One may use the formal MorseNovikov theory of this one-form over $\mathcal{L}_{f, \Gamma}$ and the aforementioned metric to define a Floer homology for a suitable choice of coefficient $\Lambda$. We denote this Floer homology by $H F\left(f, \Gamma, X_{f} ; \Lambda\right)_{J}$. The critical points of this action oneform consists of $f$-twisted periodic orbits of the symplectic vector fields $X_{f}$, and the flow lines satisfy the perturbed Cauchy-Riemann equation. In particular, when $X_{f}=0$, we have an isomorphism between the $H F$ chain complexes and the $H P$ chain complexes for the same triple $f, \Gamma, J$. Furthermore, in the language of Section 6.1, there is a natural functor from the fundamental groupoid of $\mathcal{L}_{f, \Gamma}$ to the version of $\underline{\mathfrak{G}}$ in the periodic Floer homology, whose morphism sets are $H_{2}\left(M ; \Theta_{+}, \Theta_{-}\right)$. (This is an variant of the map $\underline{\mathrm{im}}$ defined in Section 3.1.3 of [L2], and hence we denote it by the same notation.) The functors $\mathcal{S}, \mathcal{E}_{\mu}$ in $H F$ (defined from the Conley-Zehnder index and the aforementioned action one-form) both factor through im. Thus, by restricting the choice of local systems to those which factor through this map, we may use the same groupoid as the $\underline{\mathfrak{B}}$ in $H F$ as well. To continue the comparison, recall the basic fact (6.7) on $H P$. When $X_{f}=0$, the $H P$ version of $e_{\mu}$, i.e., $\left[w_{f}\right]$, pulls back by $\underline{\mathrm{im}}$ to its counterpart in $H F$. However, the $H F$-version of $s$, which pulls back to $c_{1}\left(K^{-1}\right)$, is seemingly different from its counterpart in $H P$, namely $c_{\Gamma}=c_{1}\left(K^{-1}\right)+2 e_{\Gamma}$. However, this is inconsequential. The fact that in the degree 1 case, $\mathcal{M}_{1}\left(\Theta_{+}, \Theta_{-}\right)$consists of holomorphic cylinders implies that in both $H F$ and $H P$ of degree 1 , the relative homology classes of the flow lines used to define their differentials fall in the image of $\underline{i m}$. Let $\mathfrak{H} \subset H_{2}(M ; \mathbb{Z})$ denote the image of im as in $[\mathbf{L 2}]$. Then $e_{\Gamma} \mid \mathfrak{H}=0$.

This has the following implications: First, the relative grading of the degree 1 periodic Floer homology can be refined to take value in $\mathbb{Z} / p_{1} \mathbb{Z}$, where $p_{1} \geq p$ is the divisibility of $c_{1}\left(K^{-1}\right)$ on $\mathfrak{H} /$ Tors. Second, the $\left(s, e_{\mu}\right)$ completeness condition on the coefficient systems is weaker in the degree 1 case, since $s, e_{\mu}$ can now be viewed as a linear function from the smaller group $\mathfrak{H} \subset H_{2}(M ; \mathbb{Z})$. In particular, the monotonicity condition (6.4) requires only that $\left.\left[w_{f}\right]\right|_{\mathfrak{H}}$ is proportional to $c_{1}\left(K^{-1}\right) \mid \mathfrak{H}$. For example, if $f$ is monotone in the sense that $\left[w_{f}\right]$ and $c_{1}\left(K^{-1}\right)$ are proportional, then the periodic Floer homology with $\mathbb{Z}$-coefficients is well-defined for all $\Gamma \in \mathcal{N}$ at this $f$, and thus there is a well-defined notion of $H P_{1}(f ; \mathbb{Z})=\bigoplus_{\Gamma \in \mathcal{N}} H P(f, \Gamma ; \mathbb{Z})$, agreeing with the usual notion of $H F(f ; \mathbb{Z})$ (cf. Theorem 4.1.3 of $[\mathbf{L 2}])$. 
Finally, we compare the parameter spaces of these two Floer theories. In contrast to the view point we adopted for $H P$ in Appendix 7.1, we view the symplectomorphism $f$ as an intrinsic parameter of $H F$. The space $\mathcal{P}_{H F}=\underline{\mathcal{N}} \times \operatorname{Symp}_{h} \underline{\mathcal{X}} \times{ }_{\operatorname{Symp}_{h}} \underline{\mathcal{J}}$ parametrizes the above construction of $H F$. Here, $\underline{\mathcal{N}}, \underline{\mathcal{X}}, \mathcal{\mathcal { J }}$ are, respectively, the family versions of $\mathcal{N}, \mathcal{X}, \mathcal{J}_{K}$ parametrized by $\operatorname{Symp}_{h}\left(F, w_{F}\right)$. The connection $a$ induces connections on each of these bundles, and hence a connection on the fibered product $\mathcal{P}_{H F}$ as well.

We now describe the map from the HP-parameter space to the $H F$-parameter space over which the isomorphism between the two Floer homologies is defined. When restricted to degree $1 H_{1}(M ; \mathbb{Z})$ classes, the $H P$ parameter space is trivialized by the connection $a$ as $\mathcal{P}_{H P_{1}}=\mathcal{N} \times \widetilde{\operatorname{Symp}}_{h} \times \mathcal{J}$. As explained above, the same connection gives a trivialization of the pullback of the $H F$-parameter space over $\widetilde{\operatorname{Symp}}_{h}: \widetilde{\mathcal{P}}_{H F} \simeq \mathcal{N} \times \widetilde{\operatorname{Symp}_{h}} \times \mathcal{X}_{f_{0}} \times \mathcal{J}$. (cf. Section 3.1.5 in [L2], where it is also shown that parallel transport along the connection on $\mathcal{P}_{H F}$ yields isomorphisms among the $H F$ Floer chain complexes so parametrized).

Let $\widetilde{m}=\mathrm{id} \times \widetilde{s}_{0} \times \mathrm{id}$, where $\widetilde{s}_{0}$ denotes the zero section of $\widetilde{\operatorname{Symp}_{h}} \times X_{f_{0}} \rightarrow$ $\widehat{\operatorname{Symp}}_{h}$. (Note that the zero section $\widetilde{s_{0}}$ is non-constant relative to the trivialization induced by $a$, and depends on the choice of the lift $f_{0}$.) This is the map underlying the isomorphism between the parametrized versions of $H P_{1}$ and $H F$. Notice that by the invariance of $H F$ under the parallel transport determined by $a$, one may identify $H F$ on the zero section with a fiber $\mathcal{N} \times\left\{f_{0}\right\} \times \mathcal{X}_{f_{0}} \times \mathcal{J}$ of $\widetilde{\mathcal{P}}_{H F}$. As a consequence, one may take $m: \mathcal{N} \times \widetilde{\operatorname{Symp}}_{h} \times \mathcal{J} \rightarrow \mathcal{N} \times \mathcal{X}_{f_{0}} \times \mathcal{J}$ to be the map underlying the isomorphism between the parametrized versions of $H F$ and $H P_{1}$, where $m$ sends $(n, f, J)$ to $\left(n, \theta_{f}, J\right)$, where $f \mapsto \theta_{f}$ is as in the definition of $q$ in Appendix 7.1.

\section{References}

[BEHWZ] F. Bourgeois, Y. Eliashberg, H. Hofer, K. Wysocki and E. Zehnder, Compactness results in symplectic field theory, Geom. Topol. 7 (2003), 799-888.

[C] A. Cotton-Clay, Symplectic Floer homology of area-preserving surface diffeomorphisms, Geom. Topol. 13(5) (2009), 2619-2674.

[H1] M. Hutchings, An index inequality for embedded pseudoholomorphic curves in symplectizations, J. Eur. Math. Soc. (JEMS) 4 (2002), 313-361.

[HL] M. Hutchings and Y. J. Lee, Circle-valued Morse theory and Reidemeister torsion. Geom. Topol. 3 (1999), 369-396. 
[HS] M. Hutchings and M. Sullivan, The periodic Floer homology of a Dehn twist, Alg. Geom. Topol. 5 (2005), 301-354.

[HT1] M. Hutchings and C. H. Taubes, Gluing pseudoholomorphic curves along branched covered cylinders II, J. Sympl. Geom. 7 (2009), 29-133.

[HT2] - Gluing pseudoholomorphic curves along branched covered cylinders I, J. Sympl. Geom. 5 (2007), 42-137.

[KM] P. Kronheimer and T. Mrowka, Monopoles and three-manifolds, Cambridge University Press, Cambridge, UK, 2007.

[KT] C. Kutluhan and C. H. Taubes, The Seiberg-Witten-Floer homology and symplectic forms on $S^{1} \times M^{3}$, Geom. Topol. 13 (2009), 493-525.

[L1] Y. J. Lee, From Seiber-Witten to Gromov: manifolds with cylindrical ends, in preparation.

[L2] Y. J. Lee, Reidemeister torsion in Floer-Novikov theory and counting pseudoholomorphic tori, I. J. Sympl. Geom. 3 (2005), 221-311.

[MT] G. Meng and C. H. Taubes, $\underline{S W}=$ Milnor torsion, Math. Res. Lett. 2 (1995), 221-238.

[Q] D. Quillen, Determinants of Cauchy-Riemann operators over a Riemann surface, Funktsional Anal. i. Prilozhen. 19 (1985), 37-41.

[S] D. Salamon, Seiberg-Witten invariants of mapping tori, symplectic fixed points, and Lefschetz numbers, Turkish J. Math. 23 (1999), 117-143.

[T1] C. H. Taubes, Embedded contact homology and Seiberg-Witten-Floer cohomology I, Geom. Topol. 14 (2010), 2497-2582.

[T2] C. H. Taubes, Embedded contact homology and Seiberg-Witten-Floer cohomology II, Geom. Topol. 14 (2010), 2583-2720.

[T3] C. H. Taubes, Embedded contact homology and Seiberg-Witten-Floer cohomology III, Geom. Topol. 14 (2010), 2721-2818.

[T4] C. H. Taubes, Embedded contact homology and Seiberg-Witten-Floer cohomology IV, Geom. Topol. 14 (2010), 2819-2960.

[T5] C. H. Taubes, Embedded contact homology and Seiberg-Witten-Floer cohomology V, Geom. Topol. 14 (2010), 2961-3000.

[T6] C. H. Taubes, Seiberg-Witten and Gromov invariants for symplectic 4manifolds, International Press, Somerville, MA, 2005.

[T7] C. H. Taubes, The Seiberg-Witten equations and the Weinstein conjecture, Geom. Topol. 11 (2007), 2117-2202.

[T8] C. H. Taubes, Asymptotic spectral flow for Dirac operators, Commun. Anal. Geom. 15 (2007), 569-587.

[T9] C. H. Taubes, The Seiberg-Witten equations and the Weinstein conjecture II: More closed integral curves for the Reeb vector field, Geom. Topol. 13 (2009), 1337-1417

Department of Mathematics

Purdue University

W. LAFAYETTE, IN47907

E-mail address: yjlee@math.purdue.edu 
Department of Mathematics

HARVARD UNIVERSITY

CAMBRIDGe, MA 02138

E-mail address: chtaubes@math.harvard.edu

Received 06/26/2009, accepted 06/8/2011

The authors owe a debt of gratitude to Michael Hutchings for sharing his knowledge of periodic Floer homology and for his thoughts about the correspondence between the latter and the Seiberg-Witten-Floer homology. We also thank Andrew Cotton-Clay for pointing out Corollary 1.3 and other helpful comments.

Both authors were supported in part by NSF grants. 